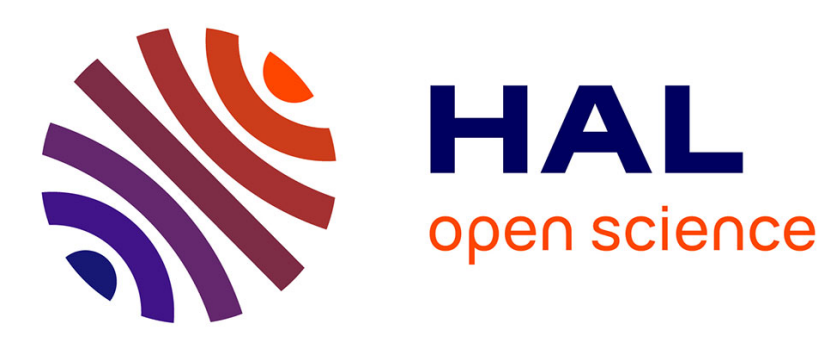

\title{
An introduction to Atomic Force Microscopy
}

Olivier Arnould

\section{To cite this version:}

Olivier Arnould. An introduction to Atomic Force Microscopy. Doctoral. Denmark. 2018. cel01955494

\section{HAL Id: cel-01955494 \\ https://hal.science/cel-01955494}

Submitted on 14 Dec 2018

HAL is a multi-disciplinary open access archive for the deposit and dissemination of scientific research documents, whether they are published or not. The documents may come from teaching and research institutions in France or abroad, or from public or private research centers.
L'archive ouverte pluridisciplinaire HAL, est destinée au dépôt et à la diffusion de documents scientifiques de niveau recherche, publiés ou non, émanant des établissements d'enseignement et de recherche français ou étrangers, des laboratoires publics ou privés. 


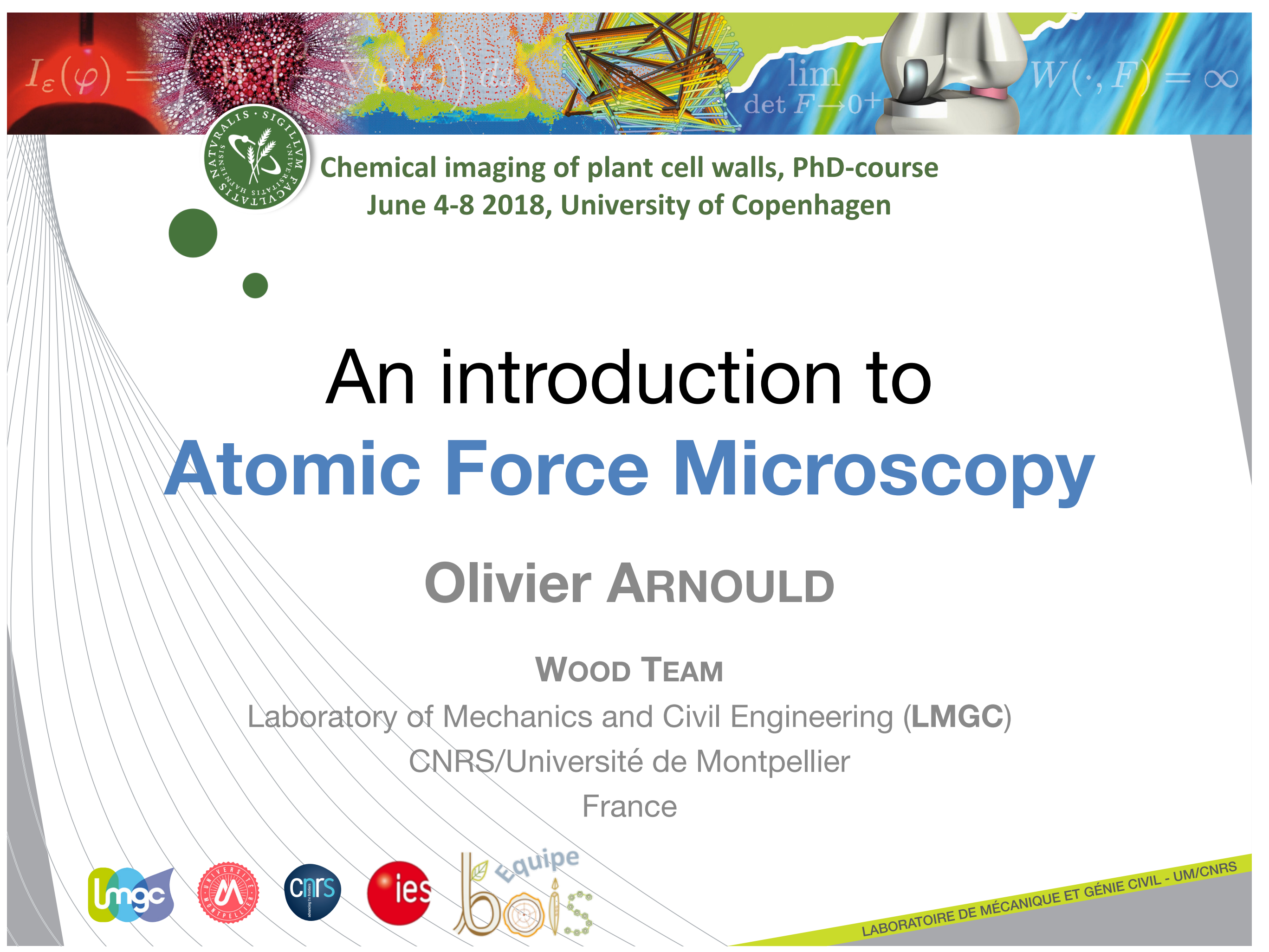


- Brief history and basic principles of scanning probe microscopy

- AFM principle and typical set-up

- Tip probe-sample surface interactions

- Tip and cantilever

- Deflection sensor and scanner

- Whole set-up

- Contact mechanics

- Operating modes

- Contact

- Intermittent

- Force-distance curve and mechanical characterisation modes Image analysis and artefacts Sample preparation 
IDEA: To measure (and use) the local (near field) probe (sharp tip)-sample interactions to map the surface morphology (topography) and physical properties

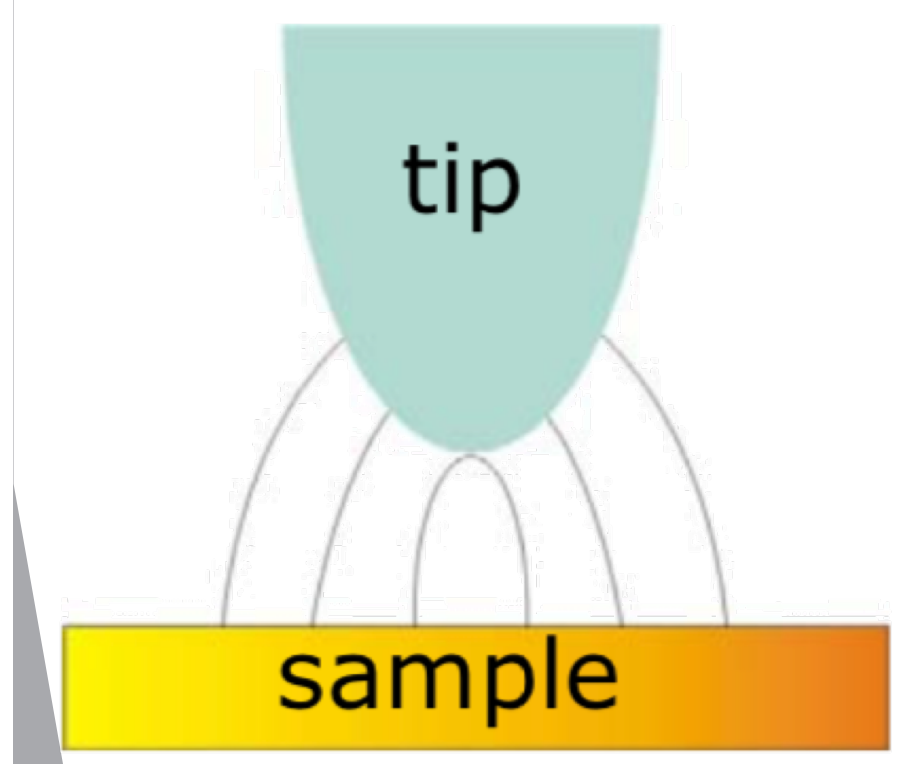

$\checkmark$ Image resolution limited by probe-sample interaction volume, not by diffraction!

$\checkmark$ Interaction can modify surface $\rightarrow$ nanolithography possible $X$ Scanning technique (quite) slow $X$ Limited maximum image size All data in SPM are subjected to interpretation 


\section{History - Forerunners}

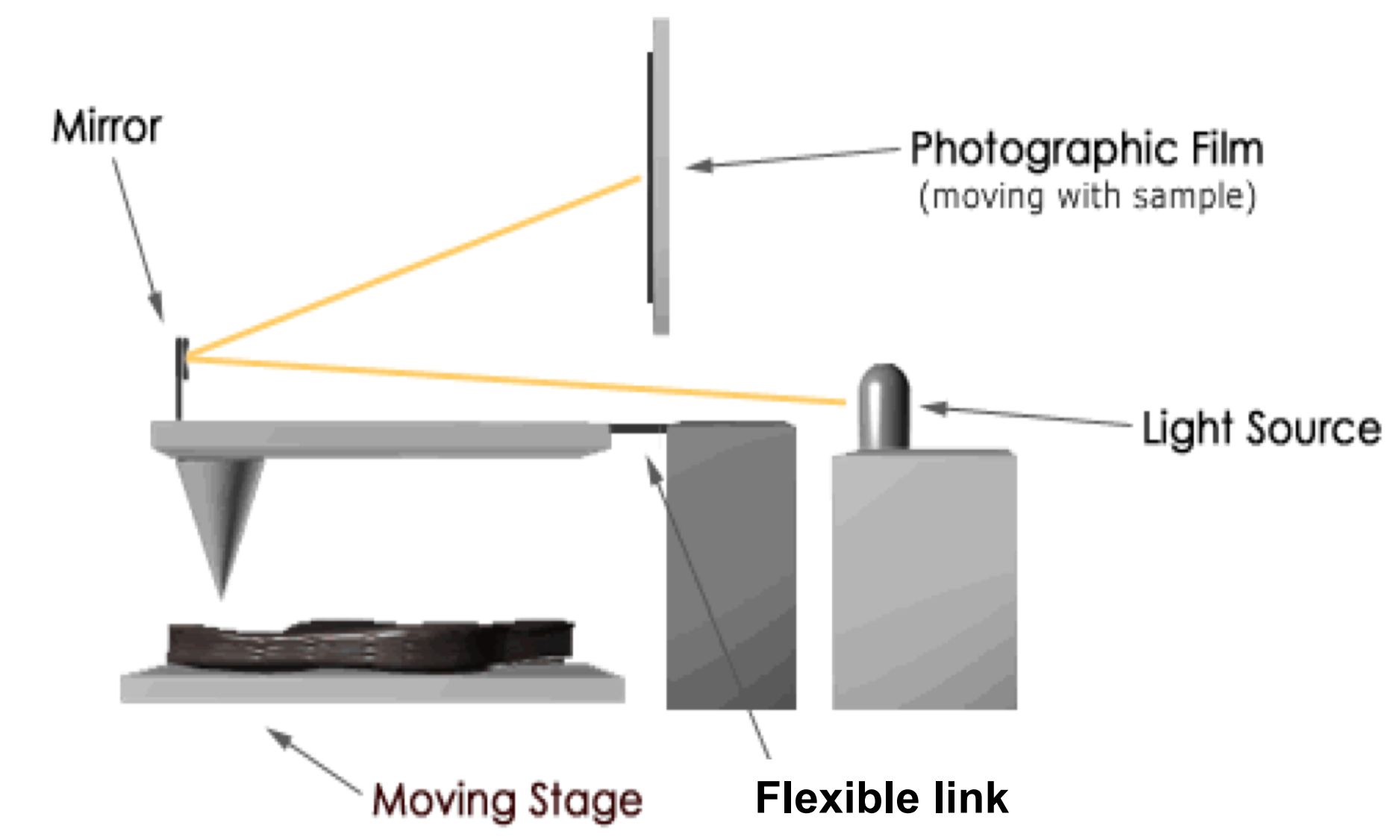

Optical stylus (optical/light lever) profilometer by Schmalz in 1929 $\approx 25 \mathrm{~nm}$ vertical resolution! (magnification $>1000 \mathrm{x}$ ) 


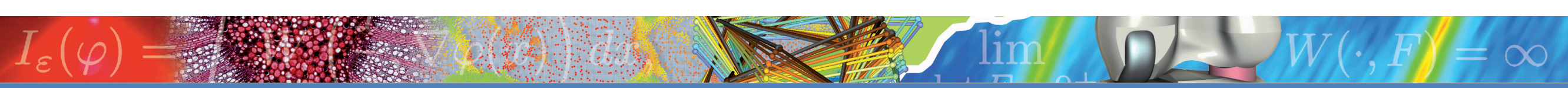

\section{History - Forerunners}

Scanning Tunnelling Microscopy (STM) by Binnig and Rohrer in 1981 (Nobel Prize in 1986)
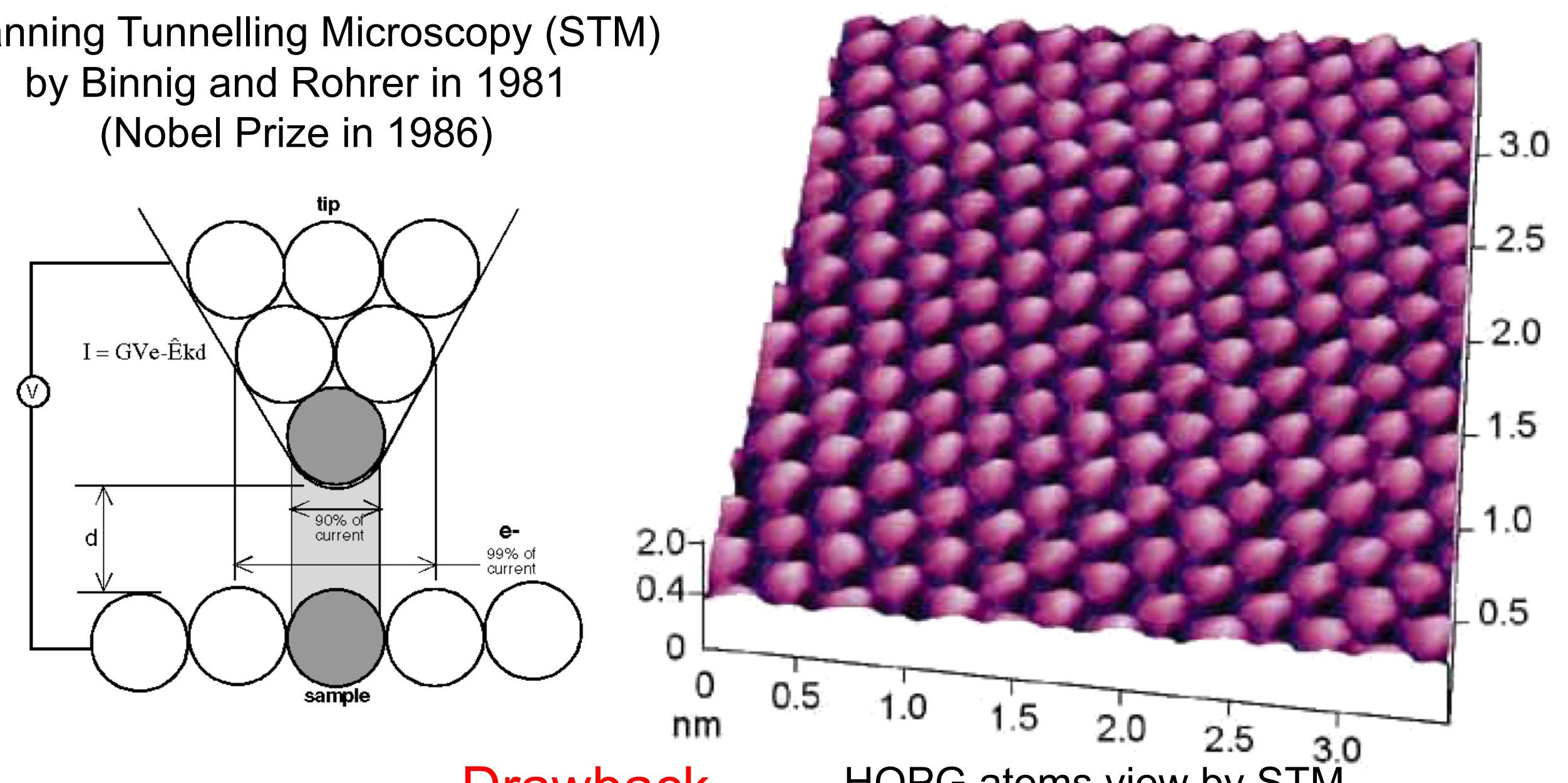

Drawback HOPG atoms view by STM need conductive material [www.ntmdt.com] and high vacuum! 


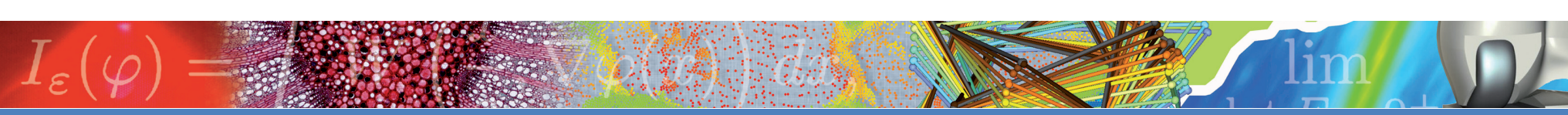

\section{History - SPM timeline}

1972 Russel Young's topografiner

1981 First STM results in the lab by Binnig, Rohrer and Gerber

1982 First publication - PRL

1984 Near field Optical Microscope

1985 Invention of AFM at Stanford by Binnig, Gerber and Quate

1986 Nobel Prize to Ruska, Binnig and Rohrer

1987 Dynamic AFN by Wickramasinghe

1987 First commerdial instruments - spin-off from Quate Group in Stan ford (Park Scientific) and Hansma Group in UCSB (Digital Instruments)

1989 IBM in atoms, py Don Eigler

1990 First microfabrifation of AFM tips by Albrecht et al.
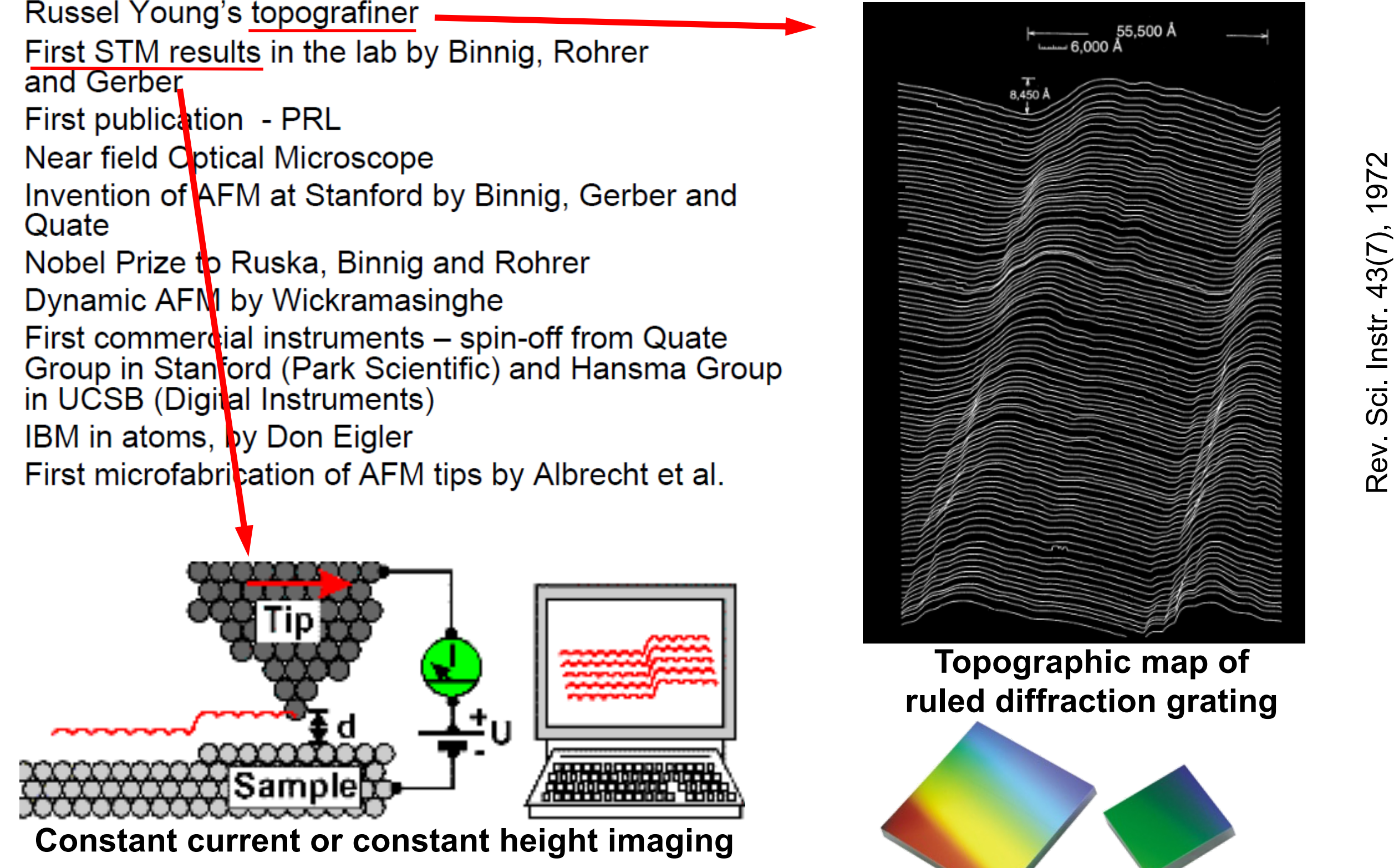

Constant current or constant height imaging 
1972 Russel Young's topografiner

1981 First STM results in the lab by Binnig, Rohrer and Gerber

1982 First publication - PRL

1984 Near field Optical Microscope

1985 Invention of AFM at Stanford by Binnig, Gerber and Quate

1986 Nobel Prize to Ruska, Binnig and Rohrer

1987 Dynamic AFM by Wickramasinghe

1987 First commercial instruments - spin-oif from Quate Group in Stanford (Park Scientific) and Hansma Group in UCSB (Digital Instruments)

1989 IBM in atoms, by Don Eigler

1990 First microfabrication of AFM tips by Albrecht et al.

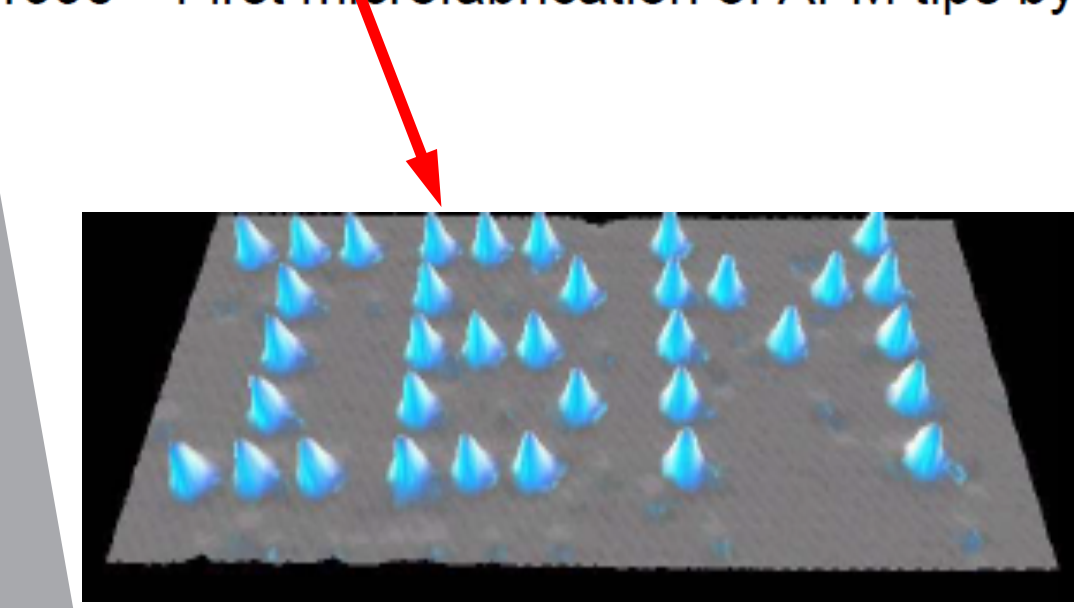

Image of Xenon atoms moved on Nickel (110) surface using an STM tip

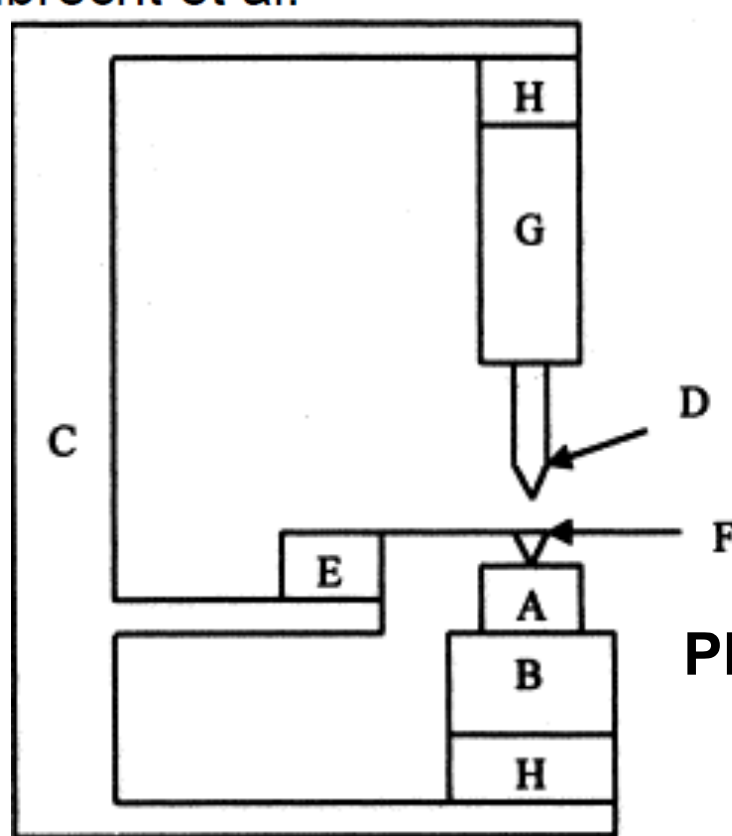

Topography of CalrSn $\mathrm{n}_{4}(110)$ GROWTH SPIRAL

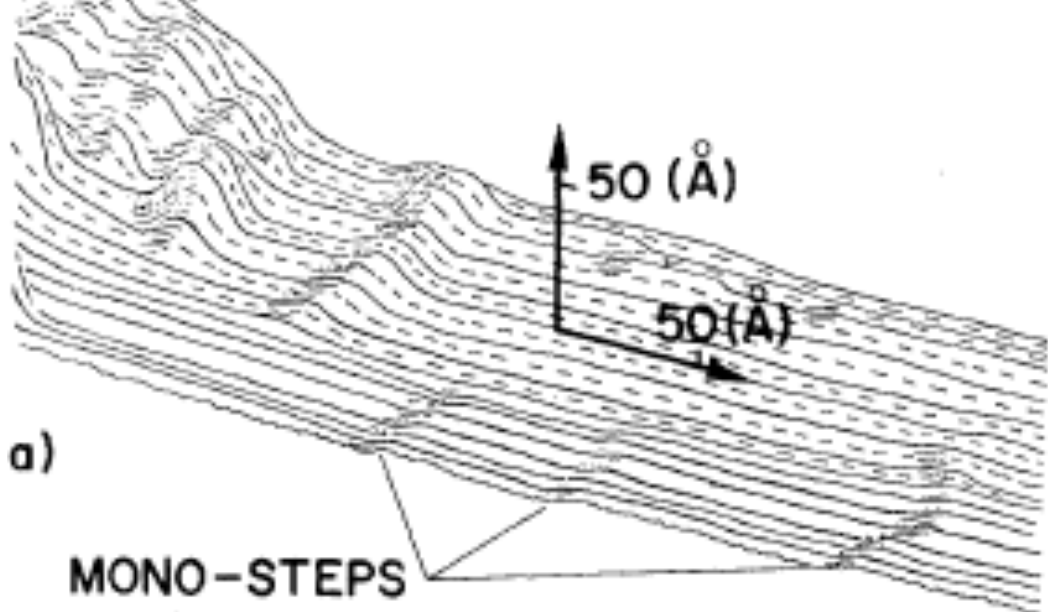

Phys. Rev. Lett., 49(1), 1982

A: Sample

B: AFM feedback and scanners

C: Aluminium block

D: STM tip (Au)

E: Modulating piezo

F: AFM tip = STM sample

G: STM feedback

$\mathrm{H}$ : Viton to damp mechanical vibrations

Original AFM design

Phys. Rev. Lett., 56 (9) (1986) 


\section{Scanning Probe Microscopy}

\section{SPM}

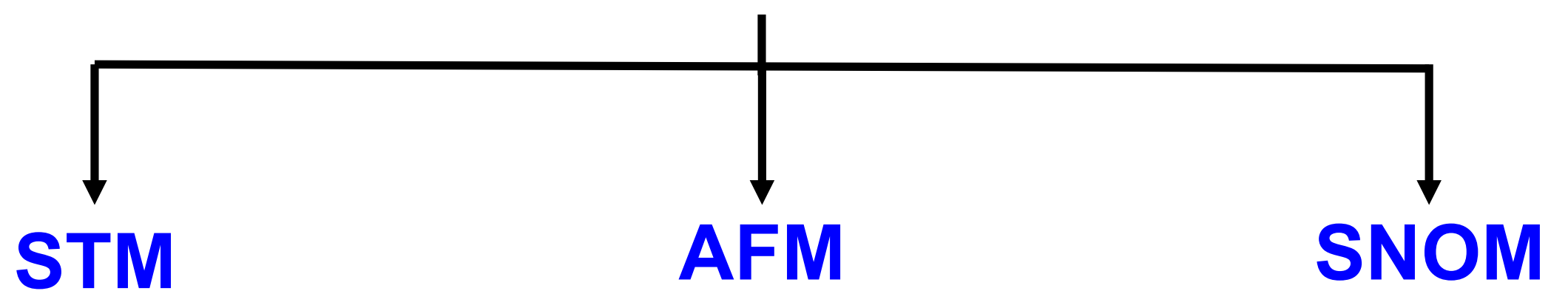

Scanning Tunneling Microscopy

Tunneling current
Atomic Force Microscopy

Local interaction forces (Van der Waals, elastic, electrostatic, friction, adhesion...)

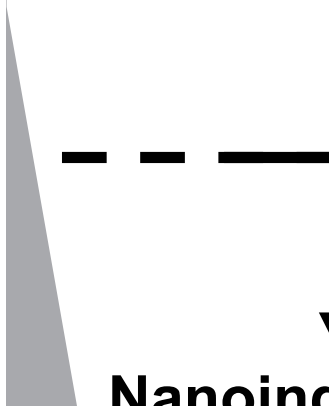

Nanoindentation Mechanical modes

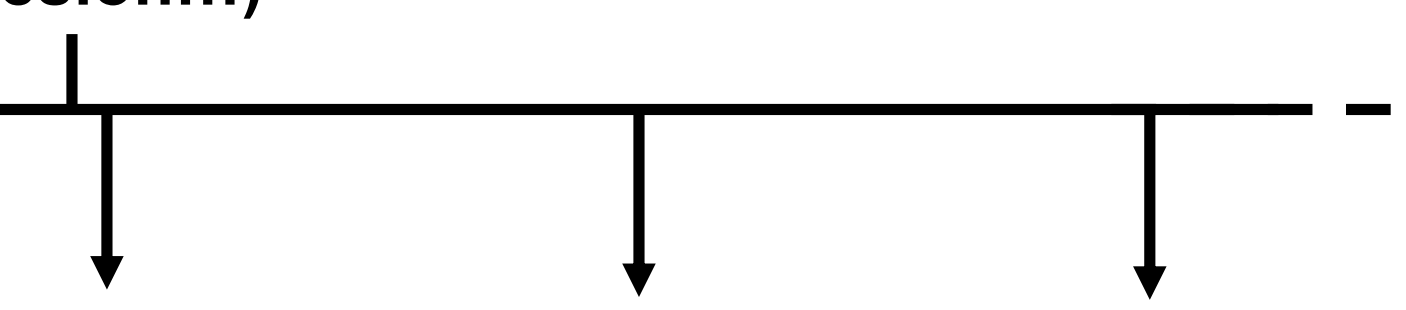

Electrostatic Conductive Scanning Thermal Chemical force Force Microscopy AFM (C-AFM) Microscopy (SThM) microscopy (EFM)
Scanning Near-field Optical Microscopy

Evanescent optical waves 


\section{Outline}

- Brief history and basic principles of scanning probe microscopy

- AFM principle and typical set-up

- Tip probe-sample surface interactions

- Tip and cantilever

- Deflection sensor and scanner

- Whole set-up

- Contact mechanics

O Operating modes

- Contact

- Intermittent

- Force-distance curve and mechanical characterisation modes

o Image analysis and artefacts

Sample preparation 


\section{Probe tip-sample interactions}

- Tip (feels the surface)

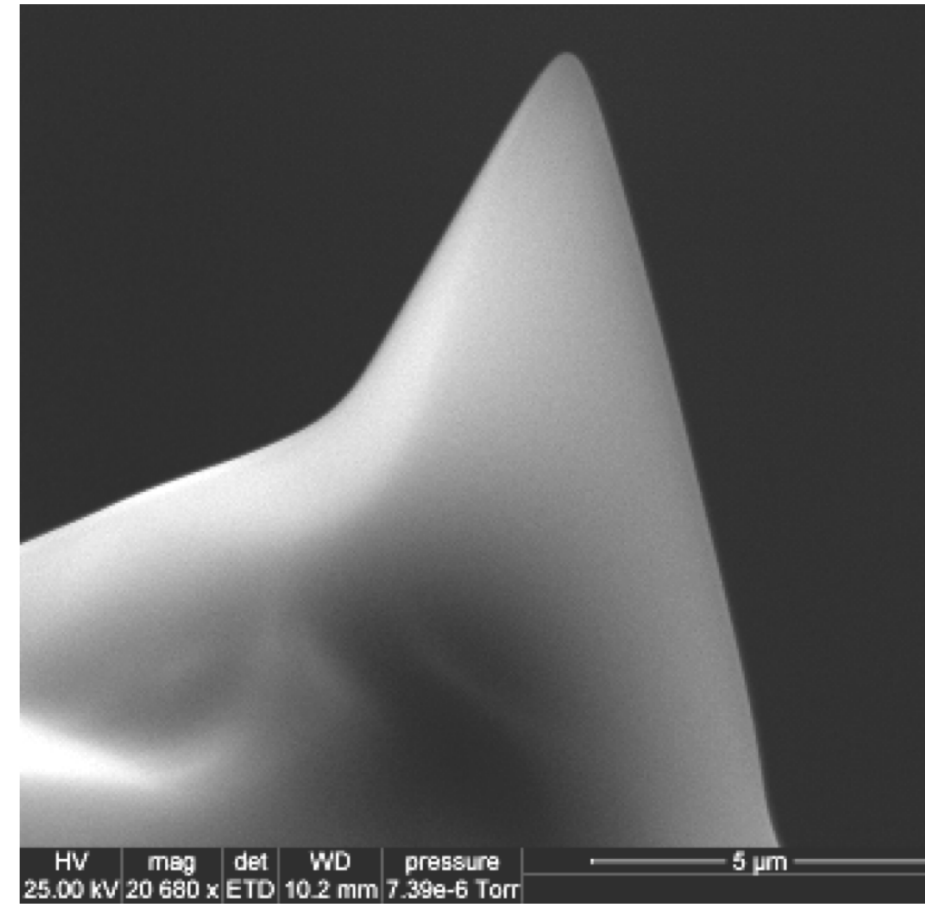

Apex radius $R \approx 2-200 \mathrm{~nm}$

Height $H \approx 10 \mu \mathrm{m}$
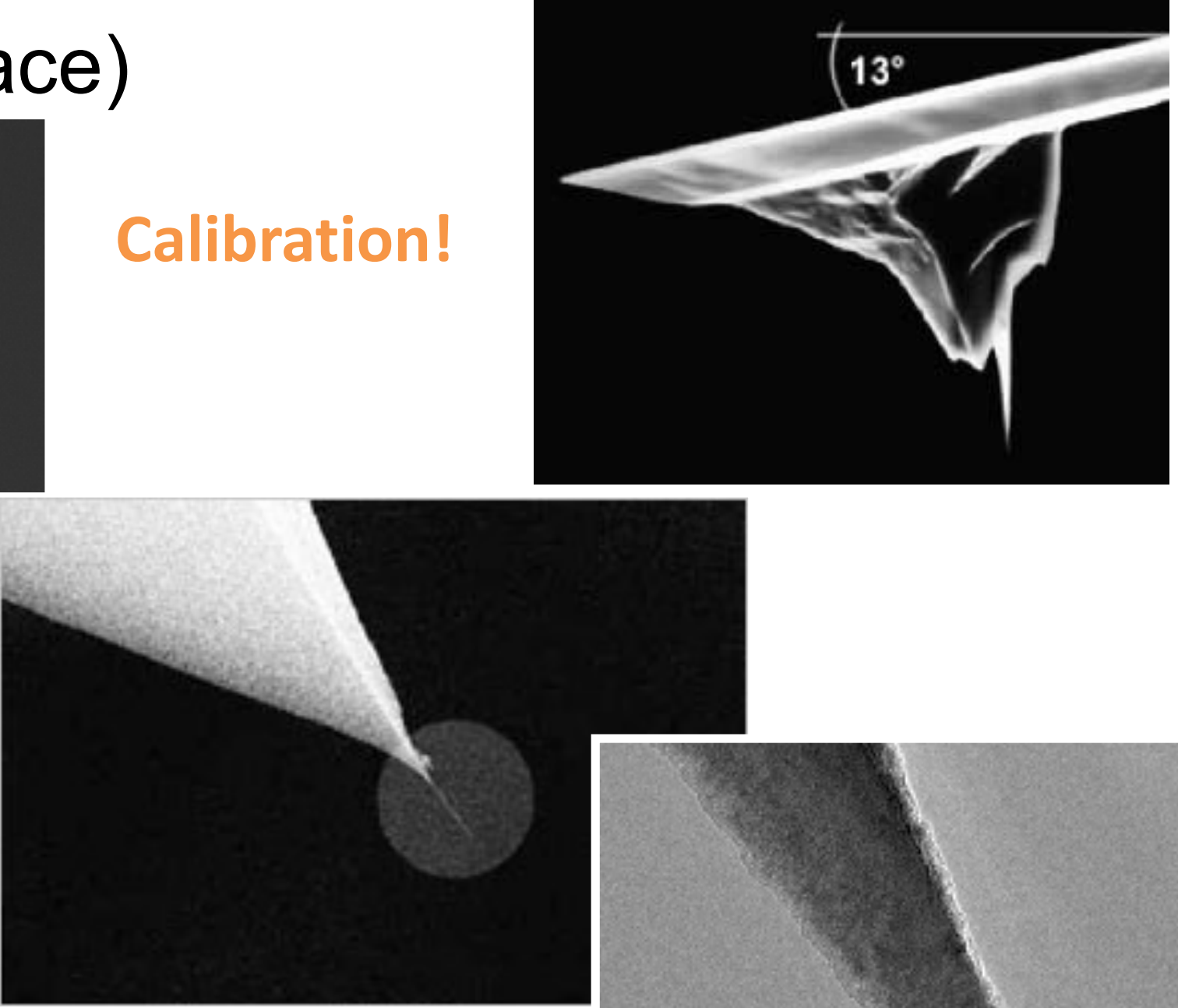

Coating (Pt, CoCr, $\mathrm{W}_{2} \mathrm{C}$, diamond, etc), functionalization, ...

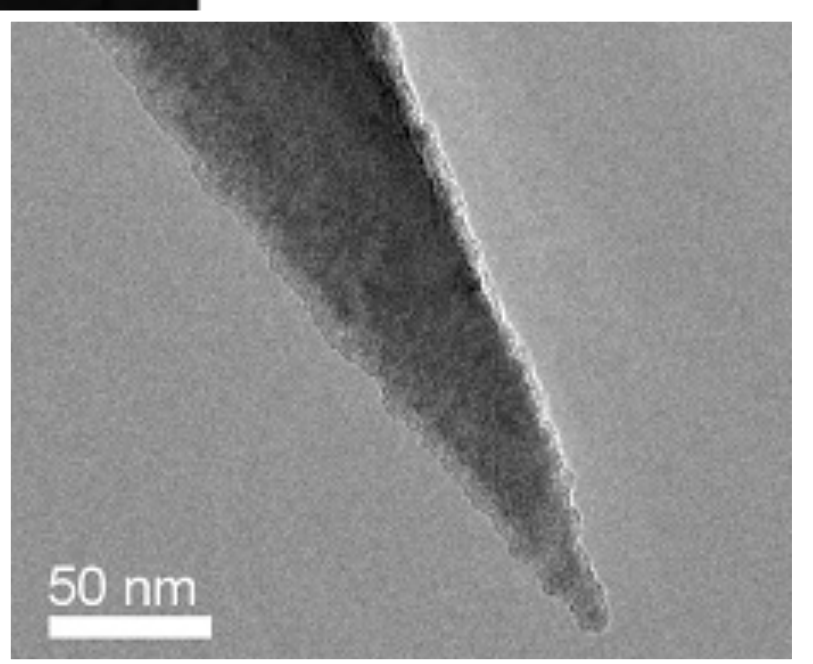




\section{Probe tip-sample interactions}

\section{The Lennard-Jones potential}

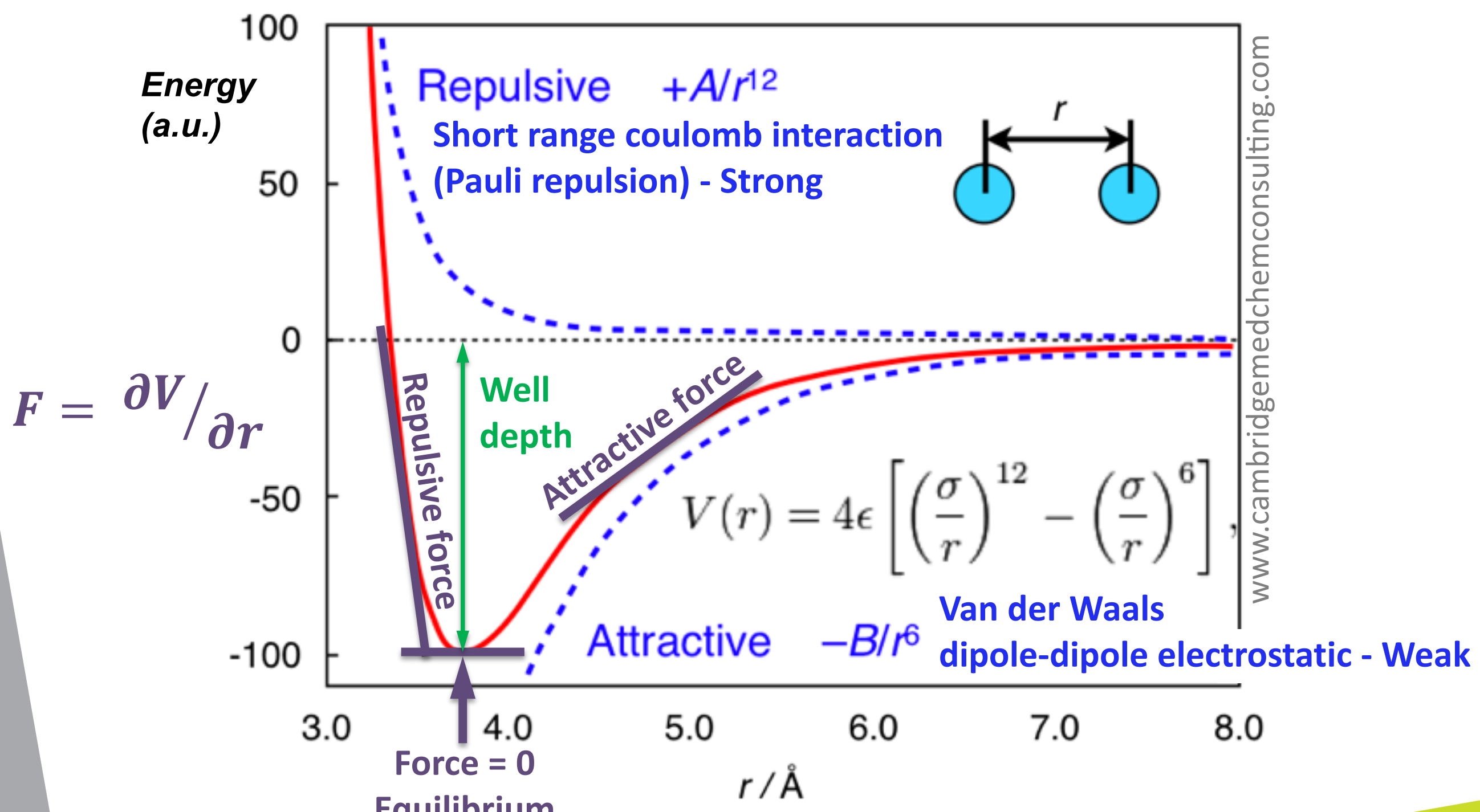

Equilibrium 


\section{Probe tip-sample interactions}

\section{Capillary force}

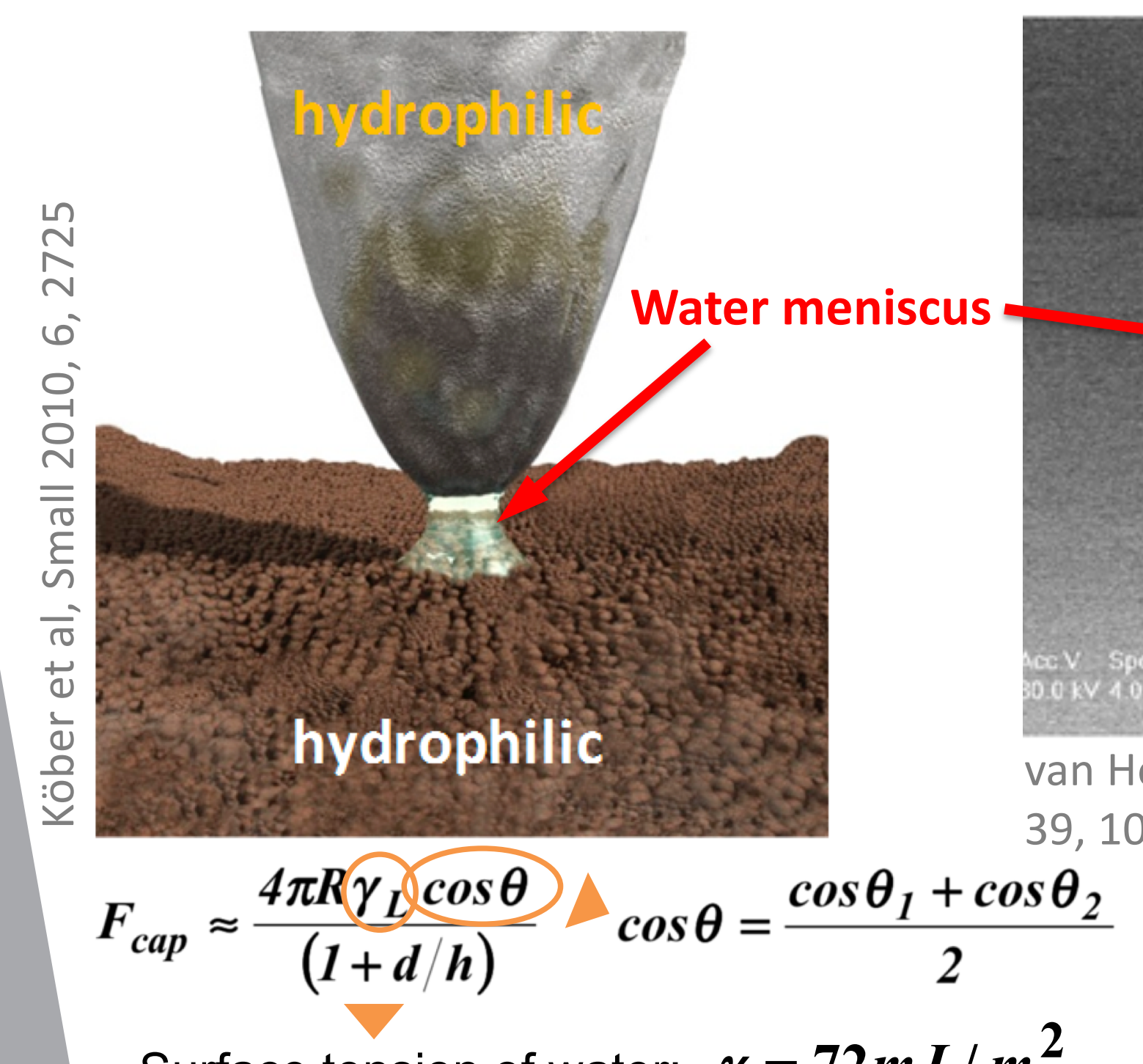

Surface tension of water: $\gamma=\mathbf{7 2 m J} / \mathrm{m}^{2}$ 


\section{Probe tip-sample interactions}

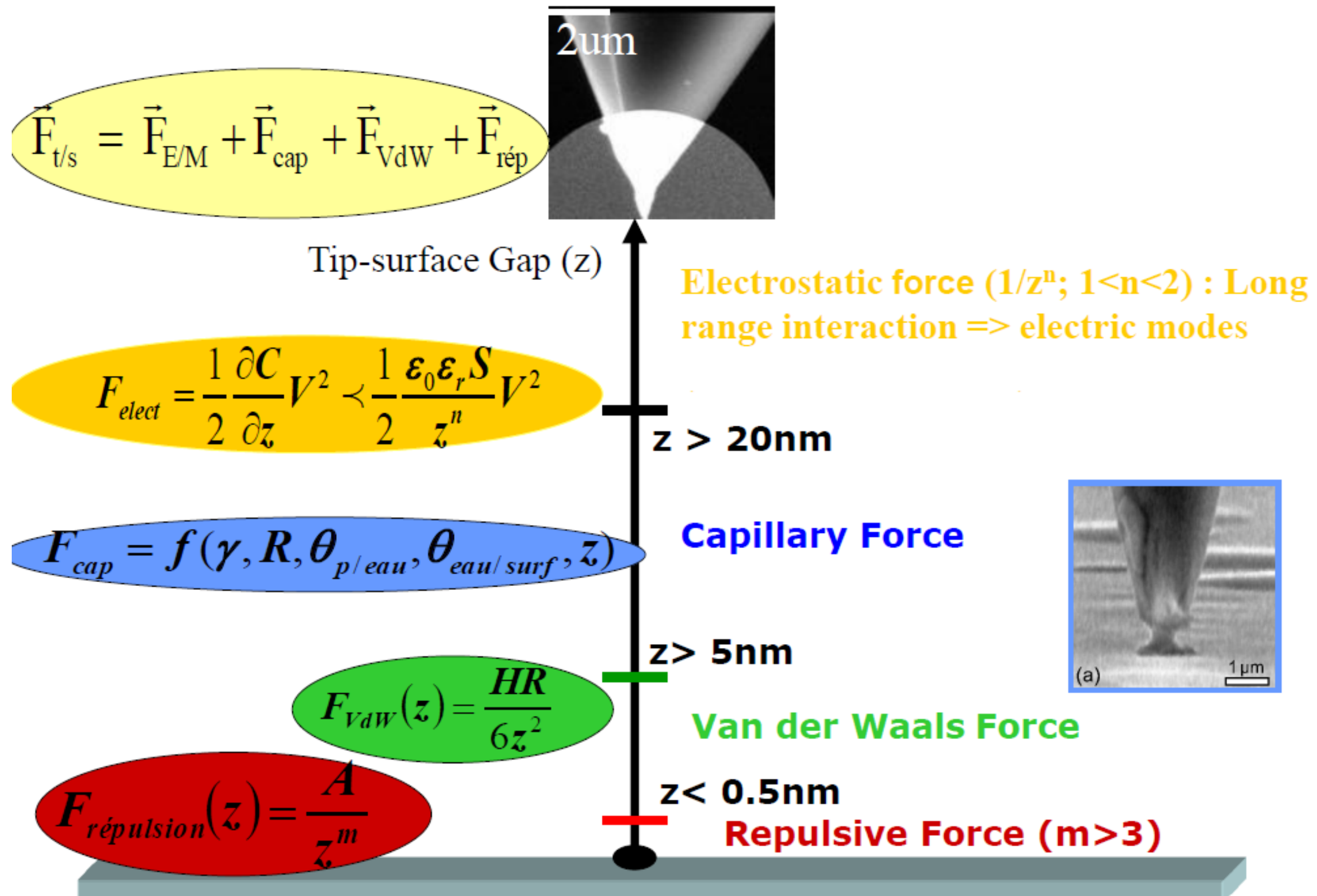


- Cantilever (spring, load sensor) Stiffness $k_{c}: 0.01-100 \mathrm{~N} / \mathrm{m}$

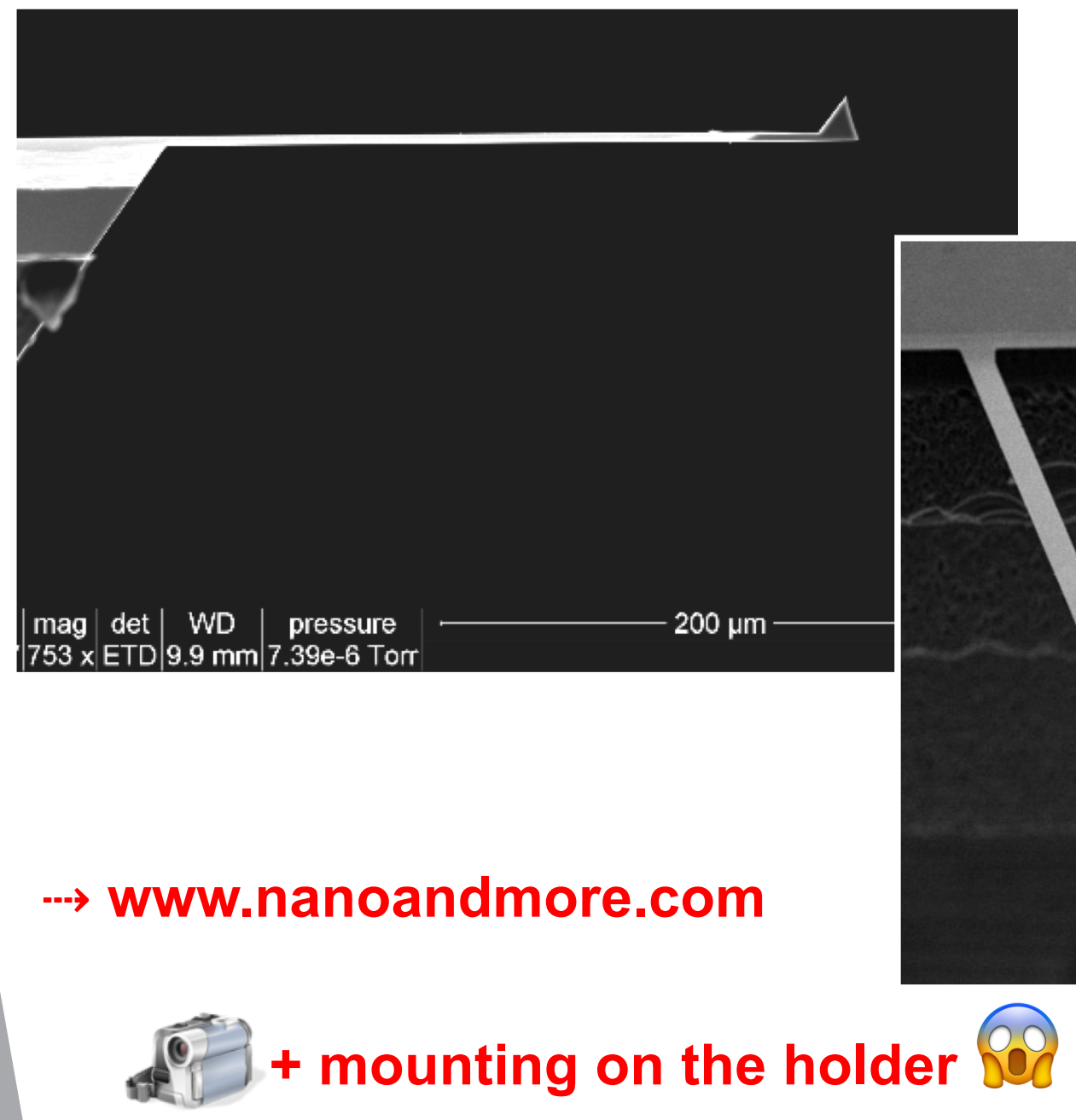

Length $L: 100-300 \mu \mathrm{m}$

Witdth $w: 10-50 \mu \mathrm{m}$

Thickness e: 0.3-4 $\mu \mathrm{m}$

mag $\mid$ det $\mid$ WD $\mid$ pressure

$200 \mu \mathrm{m}$

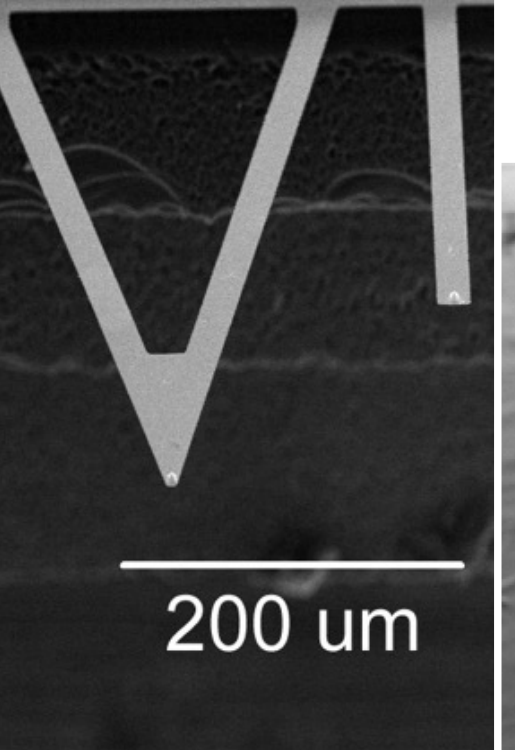

Coating

Calibration!

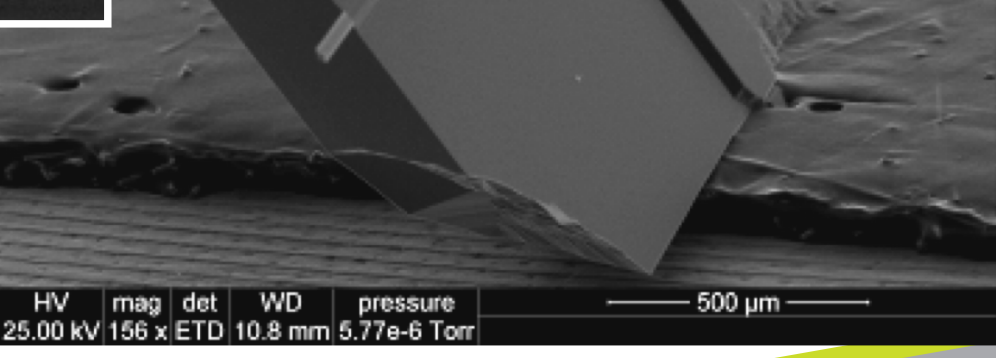


$I_{\varepsilon}(\varphi)=$
AFM principle and typical set-up

- Photodetector (deflection sensor)

Photodiode + geometry + laser position sensitivity $\approx x 10 \mathrm{~nm} / \mathrm{V}$

$\rightarrow \rightarrow$ minimum measurable deflection $<0.1 \mathrm{~nm}$ !
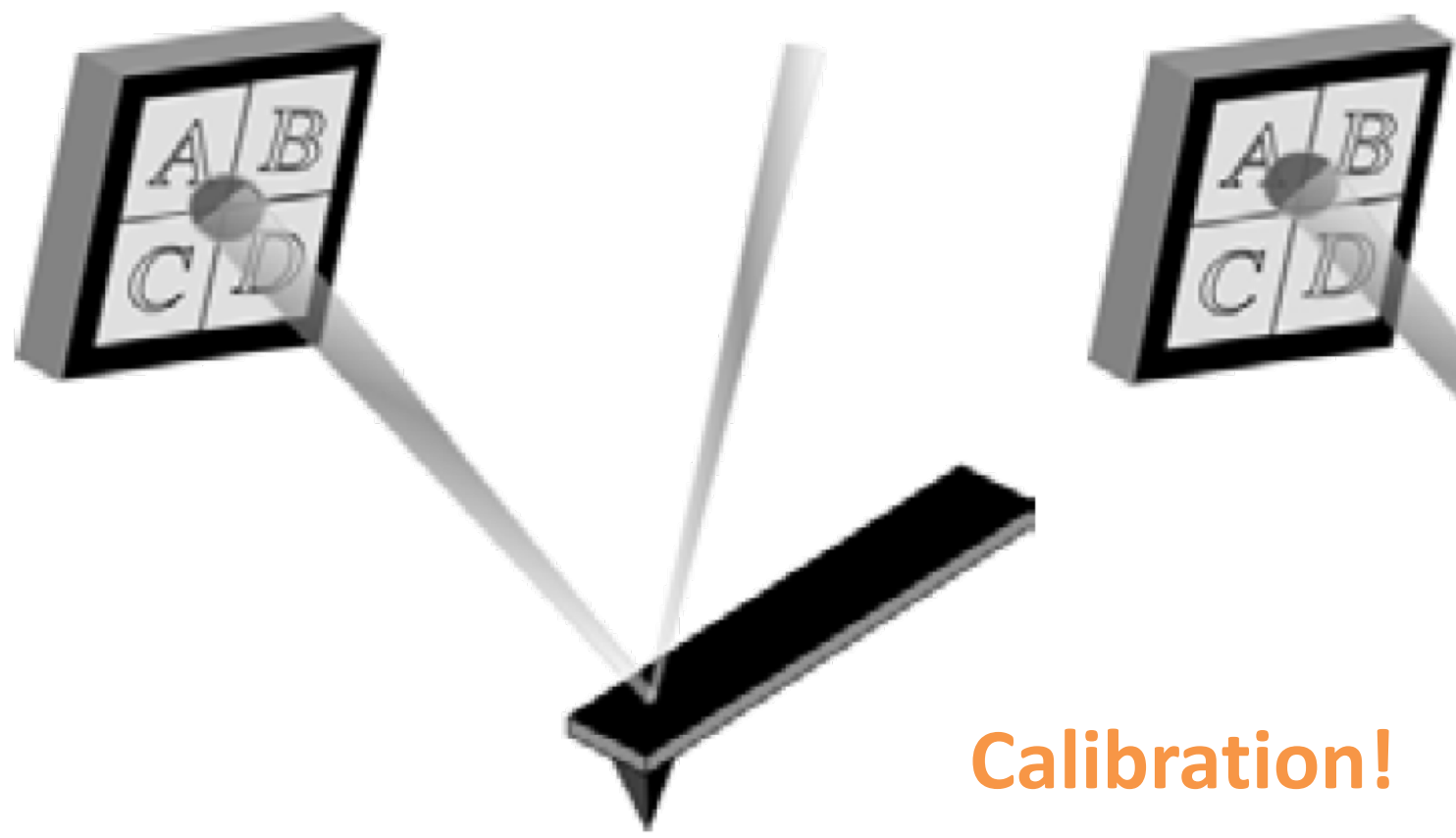

Calibration!

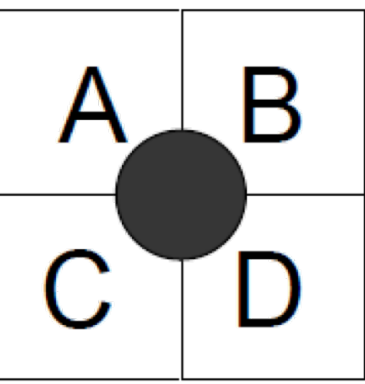

$(A+B)-(C+D)=0$
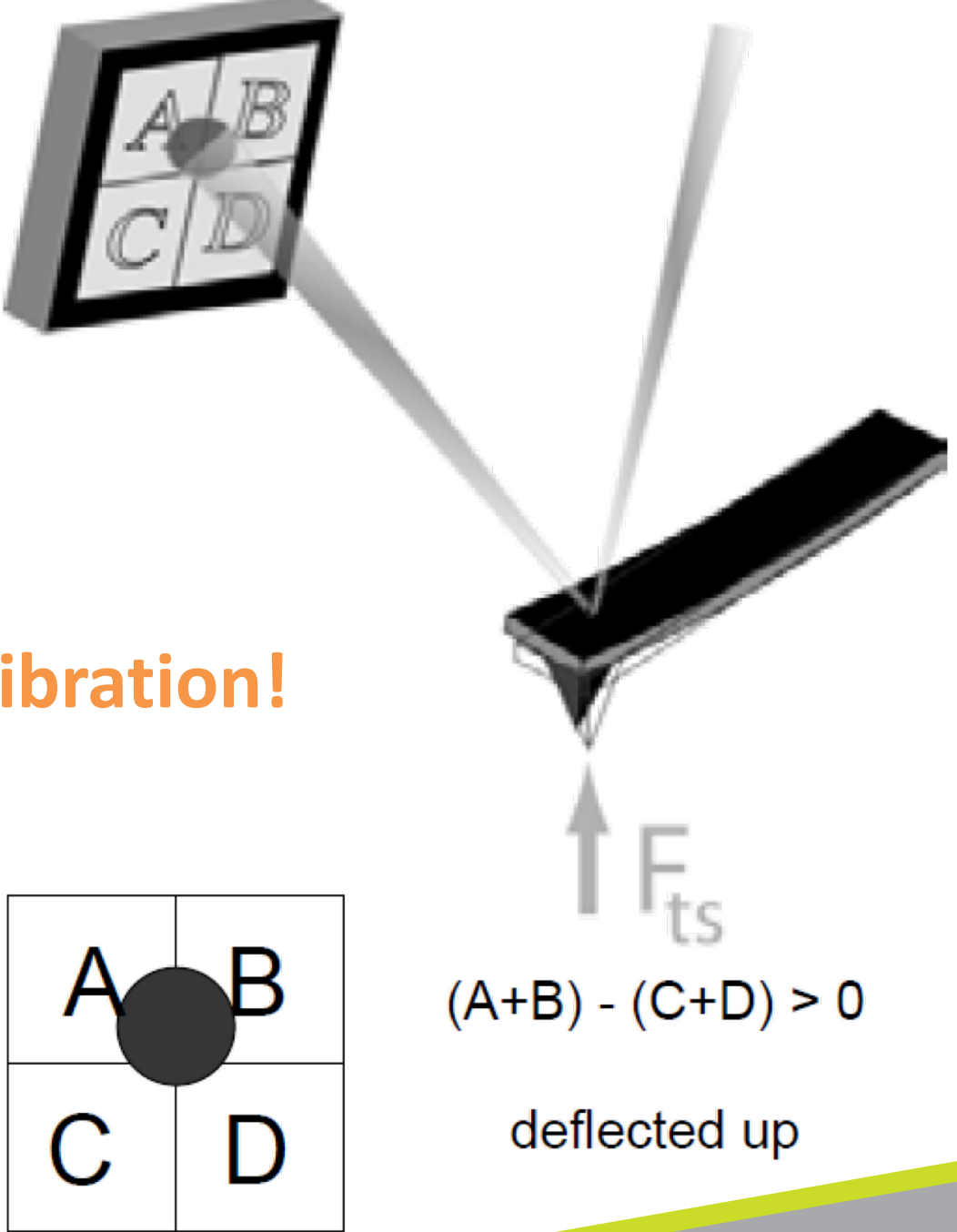

no deflection 
- Scanner

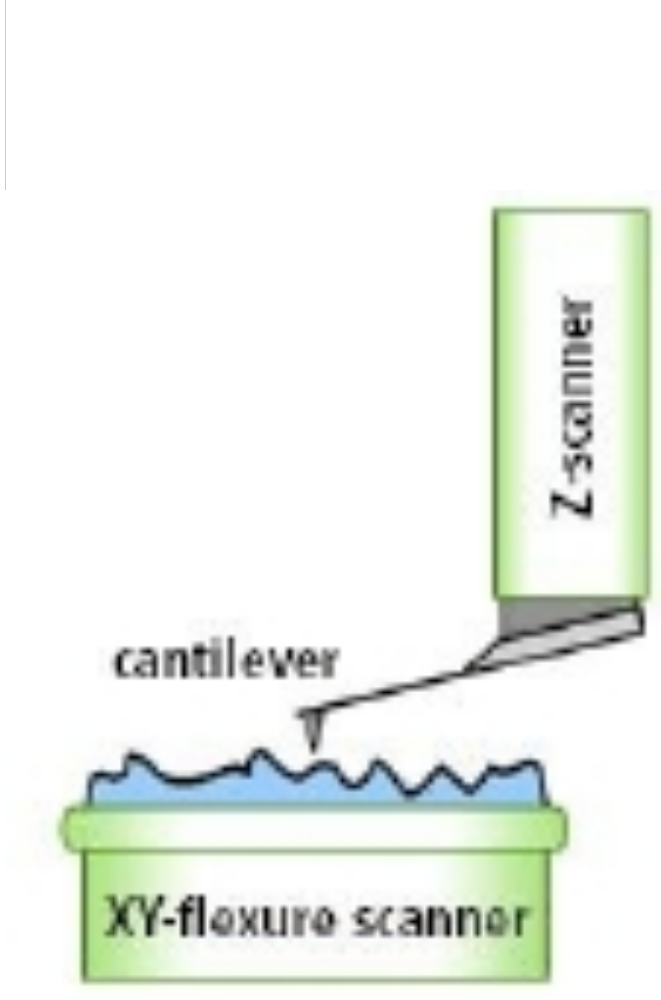
$\mathrm{mV}$ control $\rightarrow$ displacement step $<0.1 \mathrm{~nm}$ !

Calibration!

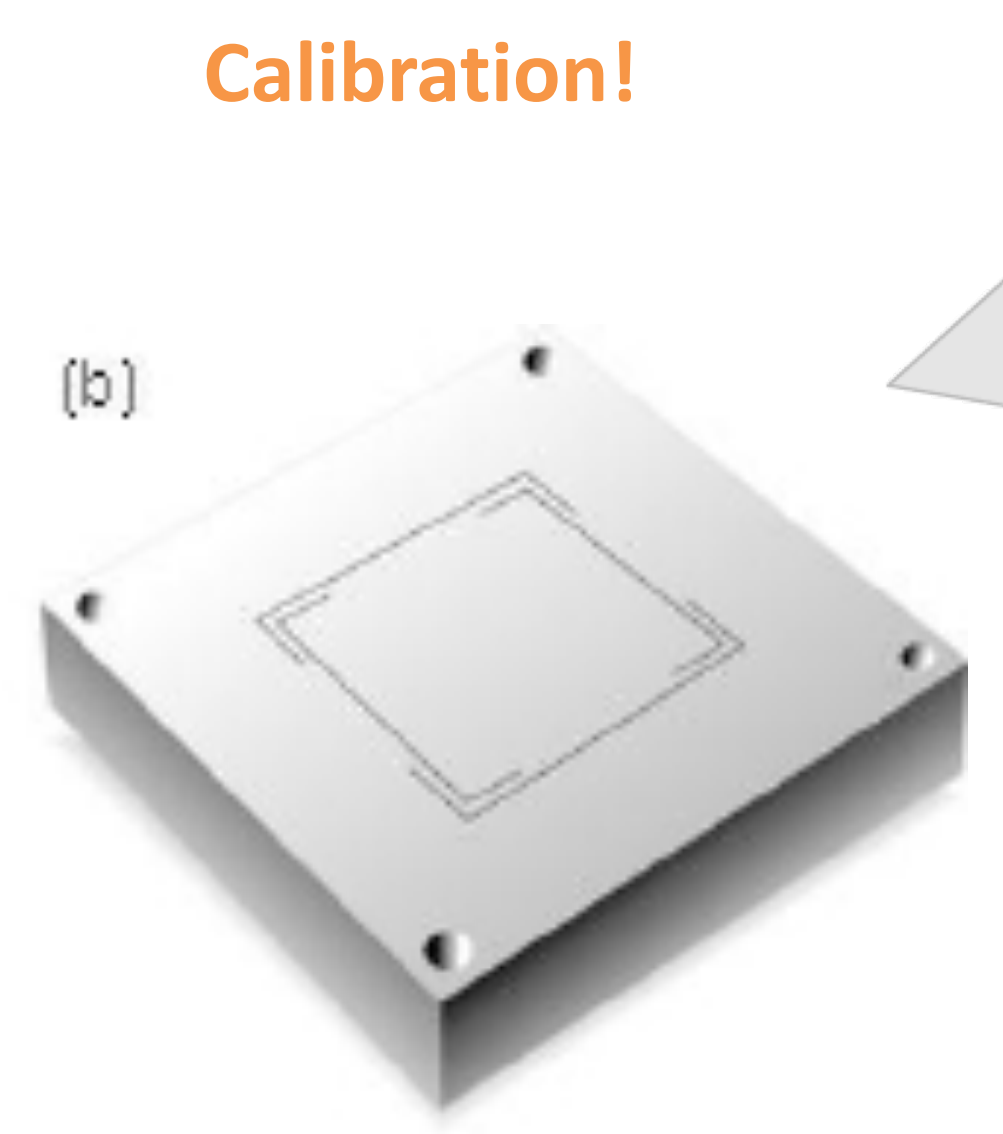

\begin{abstract}
[www.parkafm.com]
Typical piezo-response $\approx 0.1 \mathrm{~nm} / \mathrm{V}(0.01-10 \mathrm{~nm} / \mathrm{V})$
\end{abstract}

[www.veeco.com] 


\section{Trace/Retrace}

Slow-scan axis

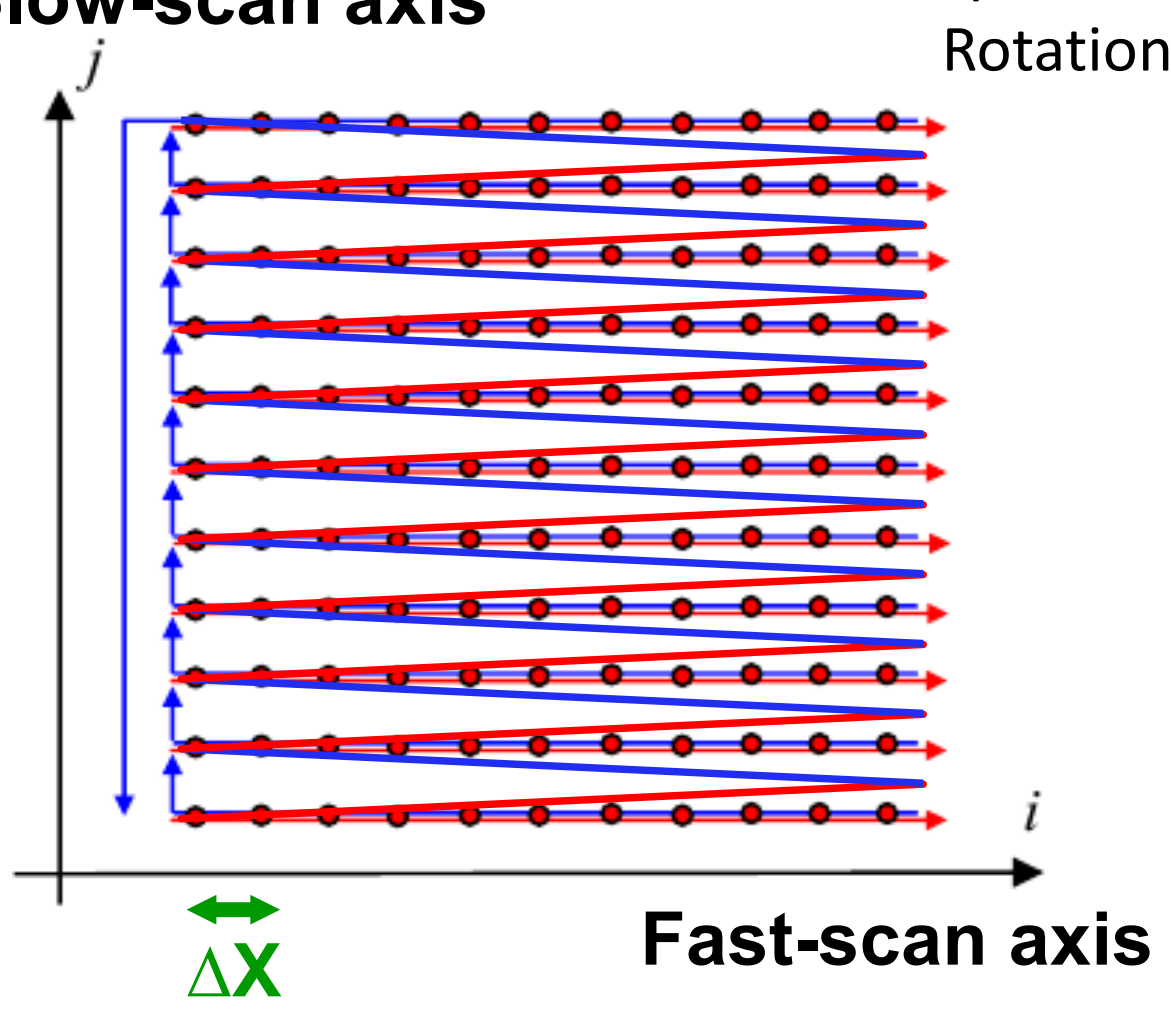

The information collected by the SPM is stored as a two-dimensional file of integer numbers $a_{i j}$ (square matrix in general)

Commonly $256 \times 256$ or $512 \times 512$ elements

The image spatial resolution is conditionned by the digitalisation

$$
\text { Example: } \quad \begin{aligned}
& \text { Image size } 10 \mu \mathrm{m} \\
& 512 \times 512 \text { elements }
\end{aligned}
$$




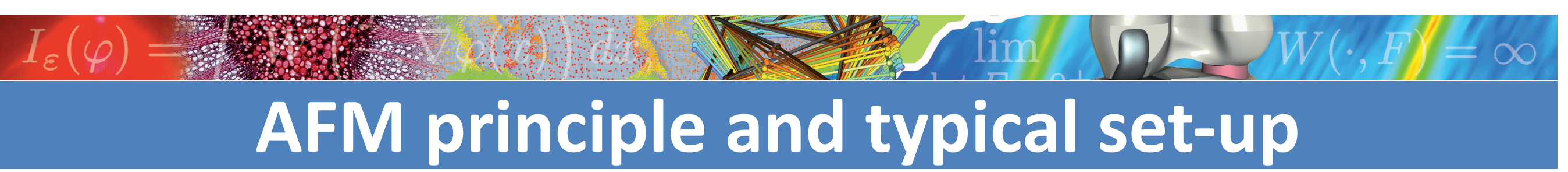

- Whole set-up

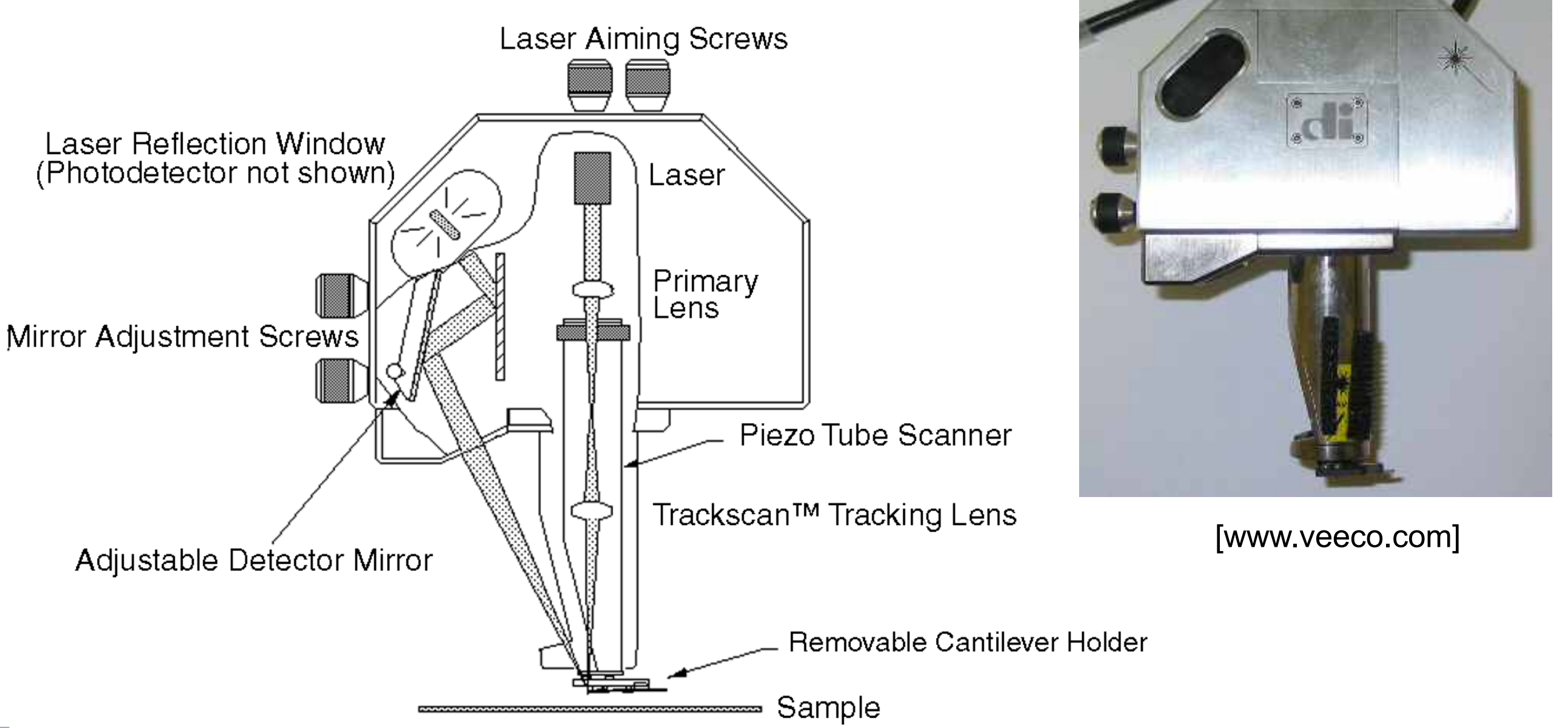




\section{Outline}

- Brief history and basic principles of scanning probe microscopy

- AFM principle and typical set-up

- Tip probe-sample surface interactions

- Tip and cantilever

- Deflection sensor and scanner

- Whole set-up

- Contact mechanics

- Operating modes

- Contact

- Intermittent

- Force-distance curve and mechanical characterisation modes

- Image analysis and artefacts

p Sample preparation 


\section{Contact mechanics}

\section{- (Depth-sensing) Nanoindentation}

$\rightarrow$ The depth of penetration $h(\delta !)$ is measured during load application mainly with a Berkovich indenter (3-sided pyramid)
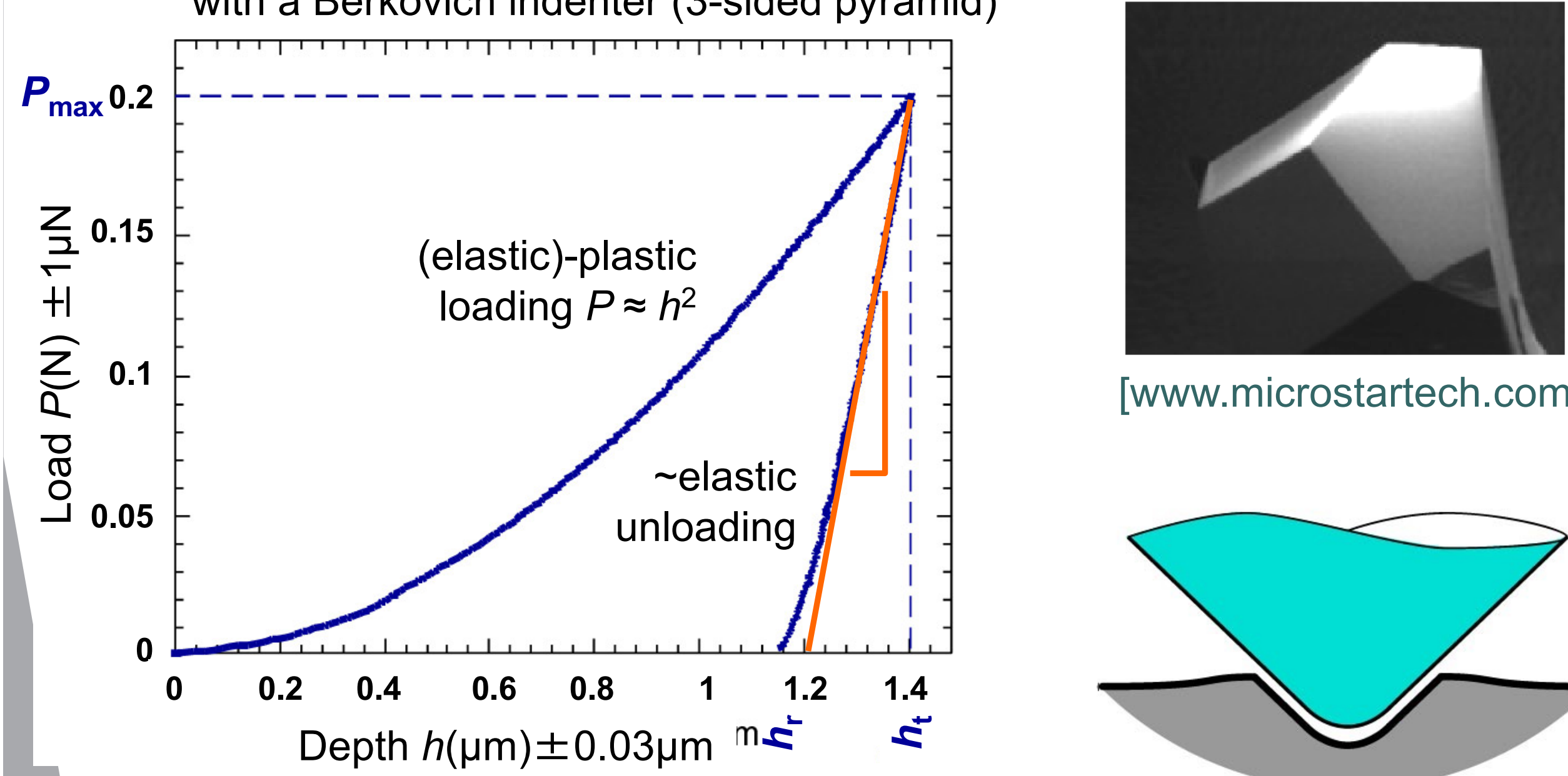

[www.microstartech.com]

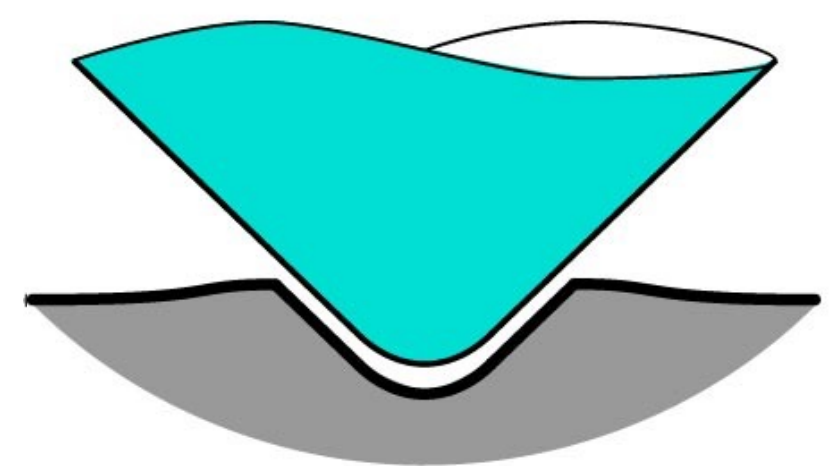

Nanoindentation curve on electrodeposited $\mathrm{Ni}$ 


\section{Contact mechanics}

- (Depth-sensing) Nanoindentation

$$
E^{*}=\frac{\sqrt{\pi}}{2} \frac{d P}{d h} \frac{1}{\sqrt{A_{c}}}
$$

With $\mathrm{E}^{*}$ or $\mathrm{M}$ reduced/contact/indentation modulus

$$
\frac{1}{E^{*}}=\frac{1-v_{i}^{2}}{E_{i}}+\frac{1-v^{2}}{E} \text { for linear ISOTROPIC }
$$

- Case of the Berkovich indenter

[A.C. Fischer-Cripps, Vacuum, 2000]

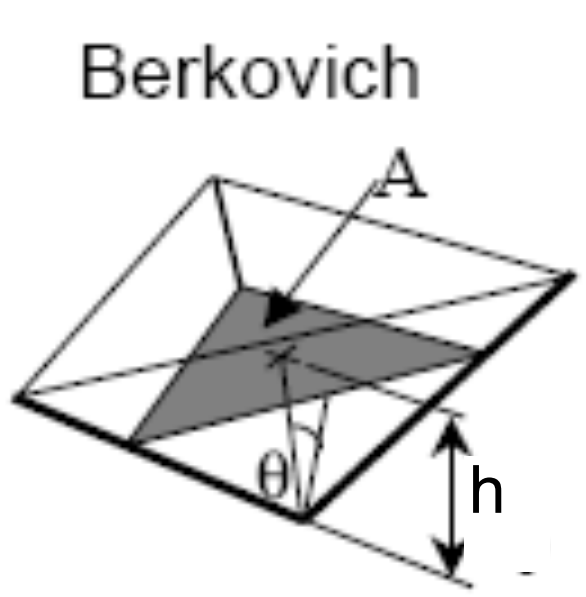

Cone
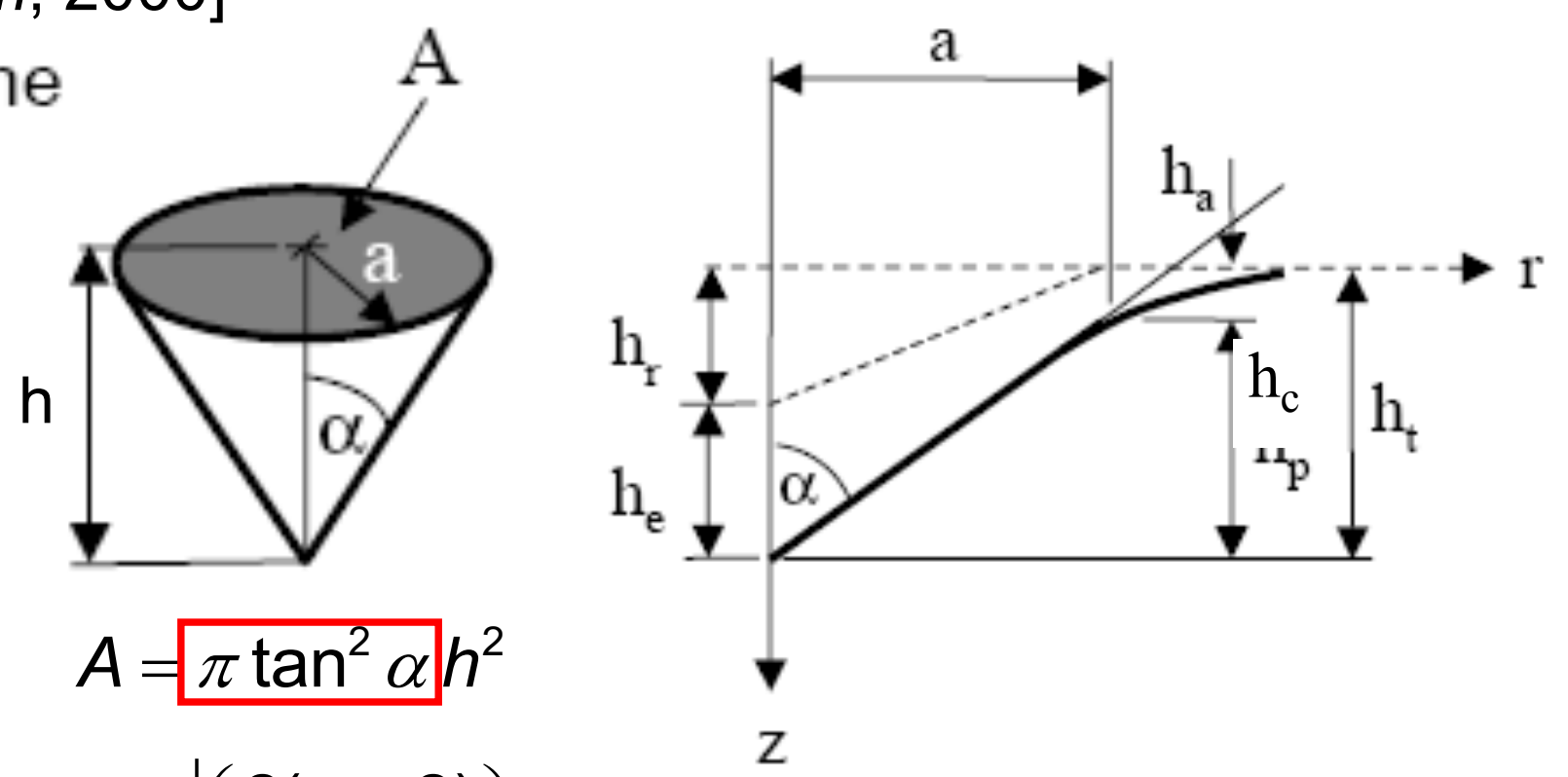

$$
h_{c}=h_{t}-\varepsilon P\left(\frac{d P}{d h}\right)^{-1} \quad \text { with } \varepsilon=\left(\begin{array}{l}
\left.\frac{2(\pi-2)}{\pi}\right) \\
0.75
\end{array}\right)
$$

cone

Berkovich 


\section{$I_{\varepsilon}(\varphi)=\gamma / 21 \%$ \\ Contact mechanics - NI vs AFM}

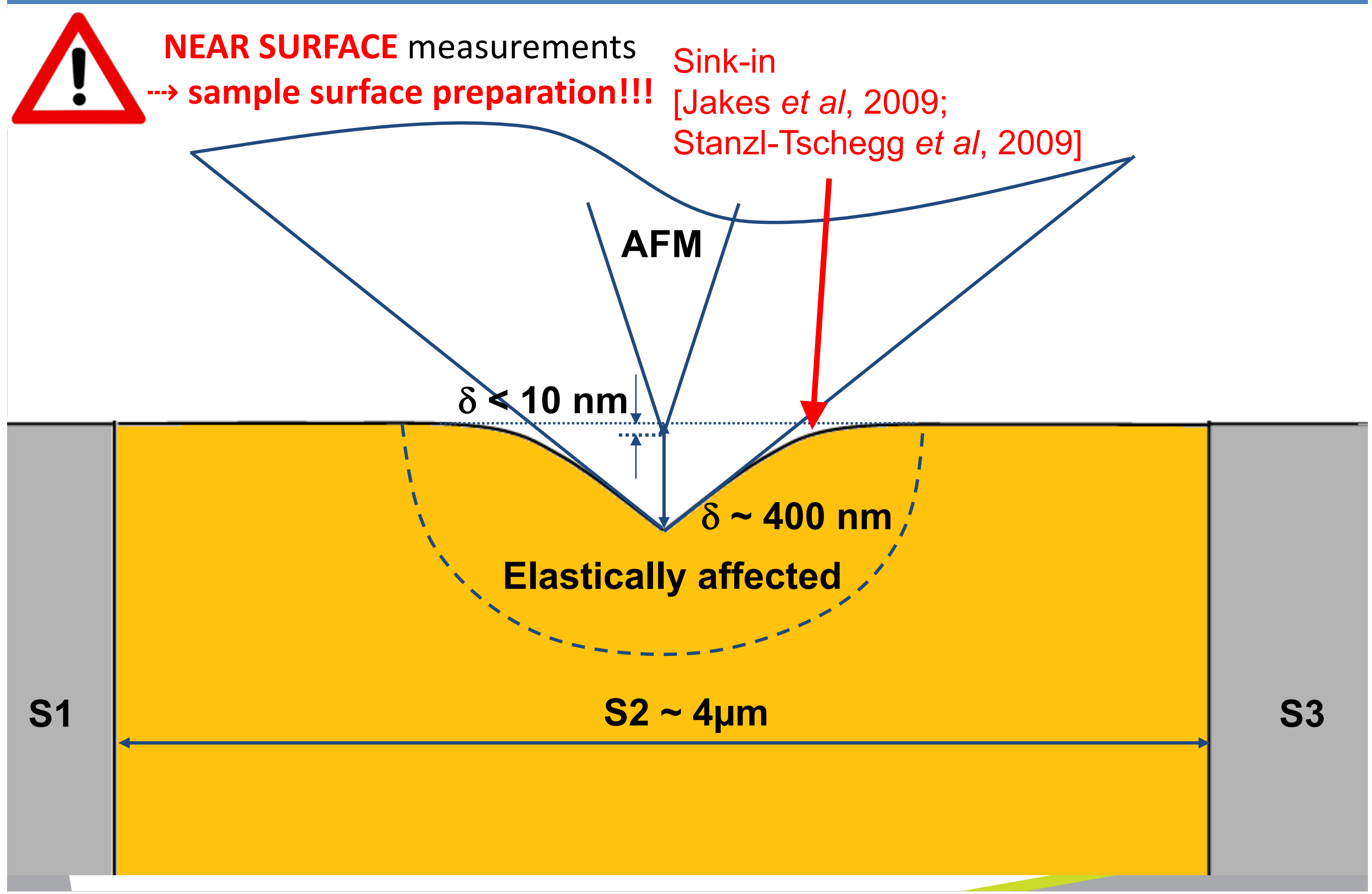


$T(6)=y_{3}$

\section{Contact mechanics without adhesion}

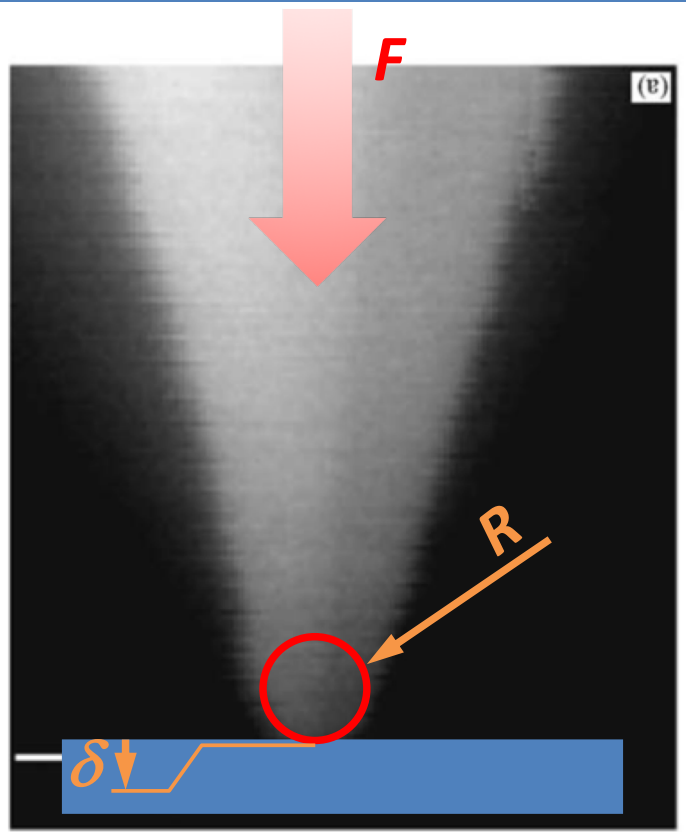

$\checkmark$ Hertz (1885)

$$
F=\frac{4}{3} M R_{e q}^{1 / 2} \delta^{3 / 2}
$$

$k_{N}=\left(6 M^{2} R_{e q} F\right)^{1 / 3}$

$$
\delta<R
$$

$\delta \approx \mathrm{R} ? ? ! !$

Non conforming

LINEAR ELASTIC HOMOGENEOUS and ISOTROPIC materials

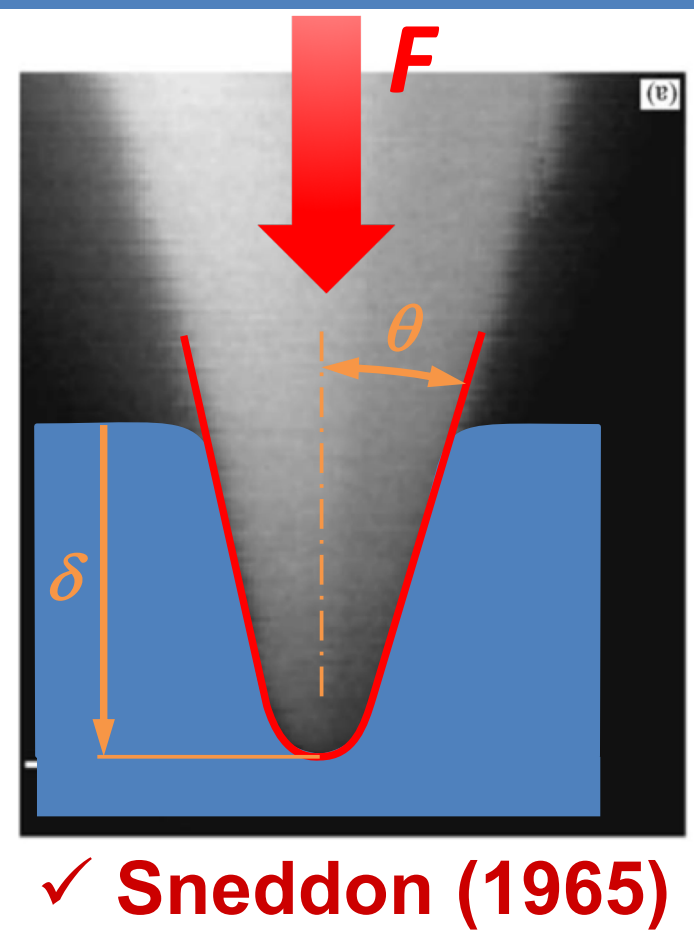

$$
F=\frac{2}{\pi} M \tan \theta \delta^{2}
$$
$\delta \gg \mathbf{R}$

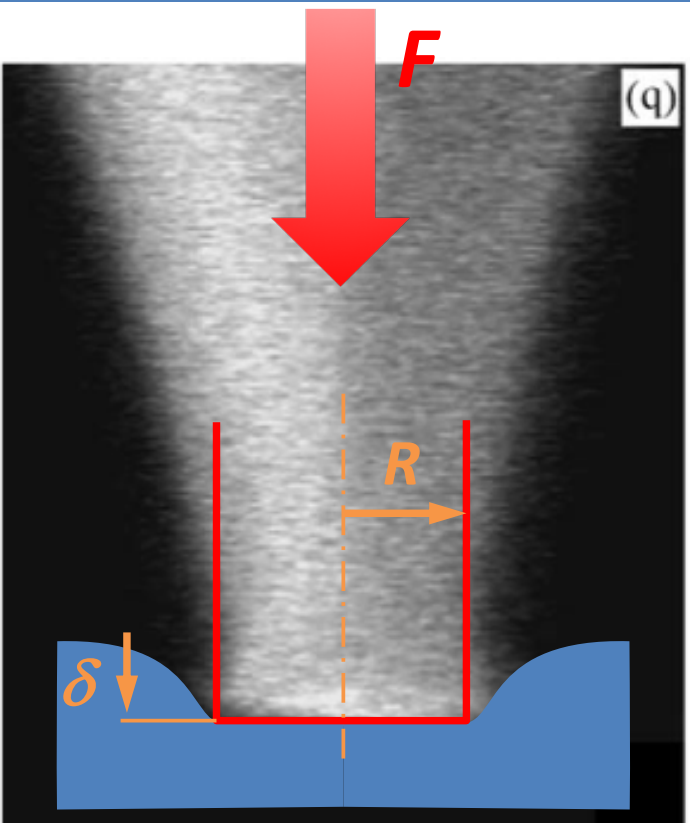

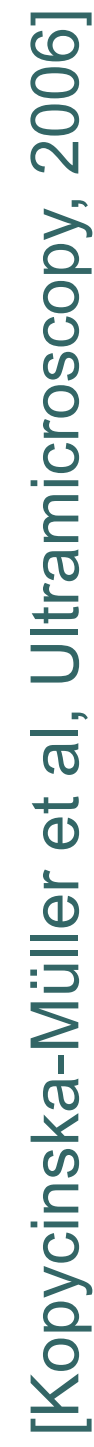

$$
F=2 M R \delta
$$

$$
k_{N}=2 M R
$$

$M$ : Reduced/contact modulus $\frac{1}{M}=\frac{1-v_{\text {Tip }}^{2}}{E_{\text {Tip }}}+\frac{1-v_{\text {Sample }}^{2}}{E_{\text {Sample }}}$ 


\section{$I_{\varepsilon}(\varphi)=8 \times x$.

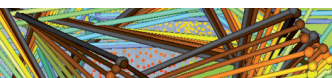 \\ Contact mechanics + adhesion (capillary, Van der Waals, ...)}

$\checkmark$ DMT Model: $\left\{\begin{array}{l}\text { Adhesive model } \\ \text { Hertz model + offset of applied load }\end{array}\right.$

$\left.k_{N_{\text {ouT }}}=\left(6 M^{2} R\left(F+F_{\text {ad }}\right)\right)\right)^{1 / 3}$ Adhesion force $\boldsymbol{F}_{\boldsymbol{a d}} \boldsymbol{d}_{\boldsymbol{D M T}}=2 \pi \boldsymbol{R} \boldsymbol{w}_{\boldsymbol{a d}}$

Rigid Plane

Used for hard samples, low adhesion force and low tip radius $\mathrm{R}$

$\checkmark$ JKR Method: $\left\{\begin{array}{l}\text { Adhesive model } \\ \text { Evaluation of the energy of adhesion between two solids }\end{array}\right.$ Hertz's theory is no longer valid: no direct relation between $F$ and $\delta$ Hertz

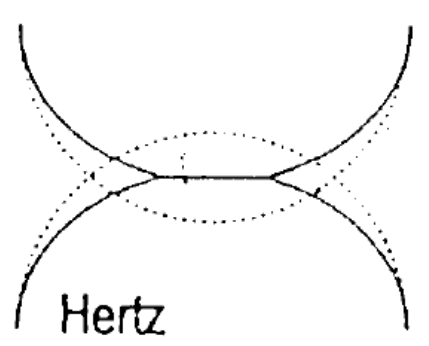

$$
k_{N_{J K R}}=2 M a_{J K R} \frac{1-\frac{1}{2}\left(\frac{a_{o_{J K R}}}{a_{J K R}}\right)^{3 / 2}}{1-\frac{1}{6}\left(\frac{a_{o_{K K R}}}{a_{J K R}}\right)^{3 / 2}}
$$

$$
\left\{\begin{array}{l}
a_{J K R}=\left[\frac{3 R}{4 M}\left(\sqrt{F_{a d_{K R}}}+\sqrt{F_{a d_{K R}}+F}\right)^{2}\right]^{1 / 3} \\
a_{0_{J K R}}=\left[\frac{3 R F_{a d_{K R}}}{M}\right]^{1 / 3} \\
F_{a d_{K K R}}=3 / 2 \pi R w_{a d}
\end{array}\right.
$$

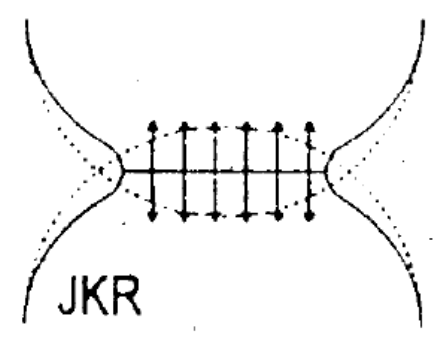

$$
\text { At pull-off } a \neq 0 \text { and } F=-F_{\text {ad }}
$$




\section{Contact mechanics}

\section{- Elastic anisotropy}

For a typical S2-layer with MFA $0^{\circ} \quad$ [Jäger et al, 2011]

$$
\begin{gathered}
M_{\mathrm{L}} \approx 19 \mathrm{GPa} \text { whereas } E_{\mathrm{L}} \approx 45 \mathrm{GPa} \\
\left(E_{\mathrm{t}} \approx E_{\mathrm{r}} \approx 12 \mathrm{GPa}, v_{\mathrm{tL}} \approx v_{\mathrm{rL}} \approx 0.028, v_{\mathrm{rt}} \approx 0.28\right. \\
\left.\mathrm{G}_{\mathrm{tL}} \approx \mathrm{G}_{\mathrm{rL}} \approx 2.5 \mathrm{GPa}, \mathrm{G}_{\mathrm{rt}} \approx 2 \mathrm{GPa}\right)
\end{gathered}
$$

Kevlar fibre: $\mathrm{M} / / \approx 15-20$ / E// $\approx 80 \mathrm{GPa}[$ Arnould et al, 2017]

in particular case...

$\rightarrow$ transversely isotropic // contact surface [Hanson, J. Appl. Mech., 1992]

$$
M_{3}=2 \sqrt{\frac{C_{11} C_{33}-C_{13}^{2}}{C_{11}}\left(\frac{1}{C_{44}}+\frac{2}{\sqrt{C_{11} C_{33}}+C_{13}}\right)^{-1}}
$$

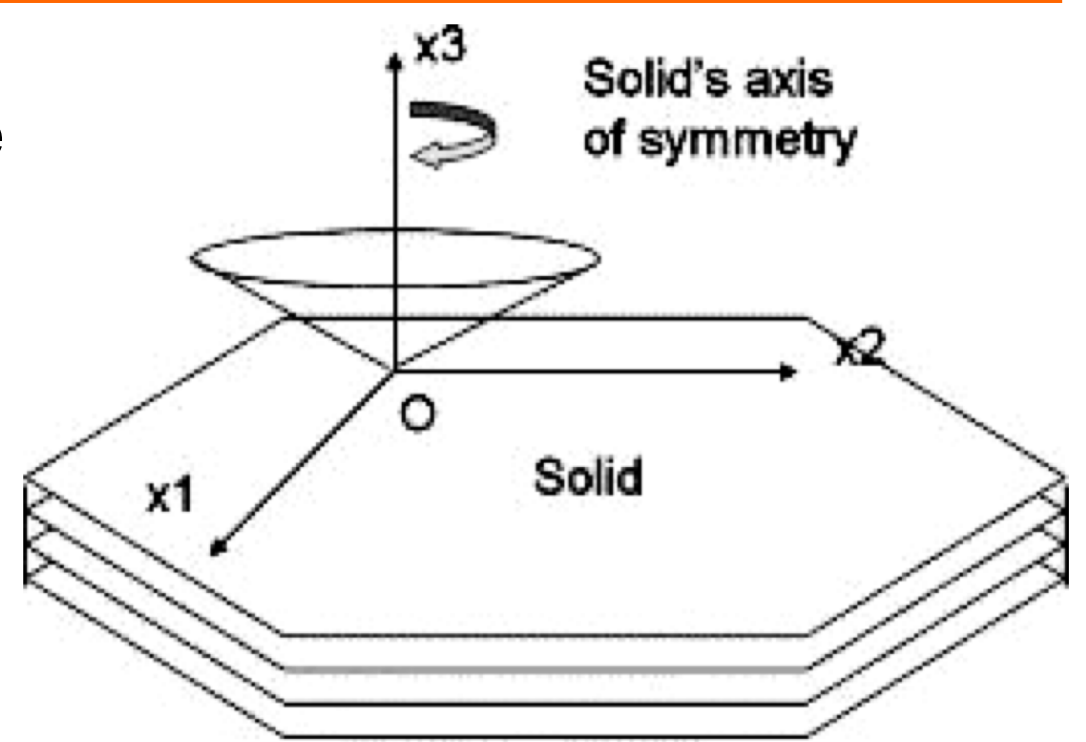




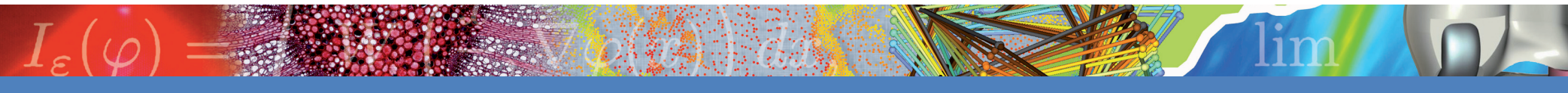 \\ Contact mechanics - Fibre cell wall anisotropy}

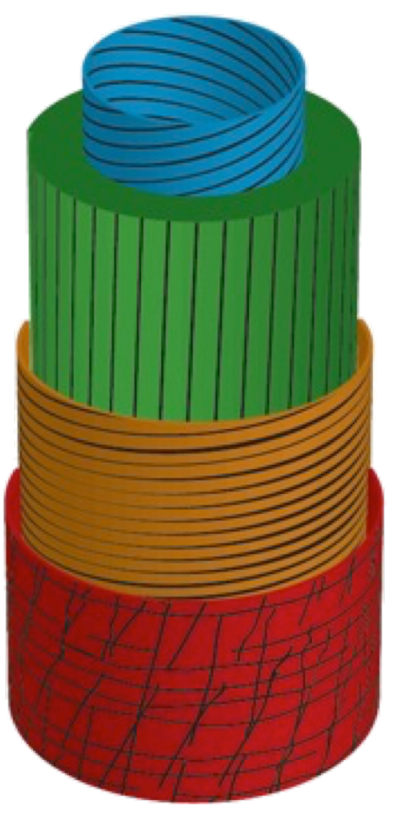

Nanoindentation on Spruce

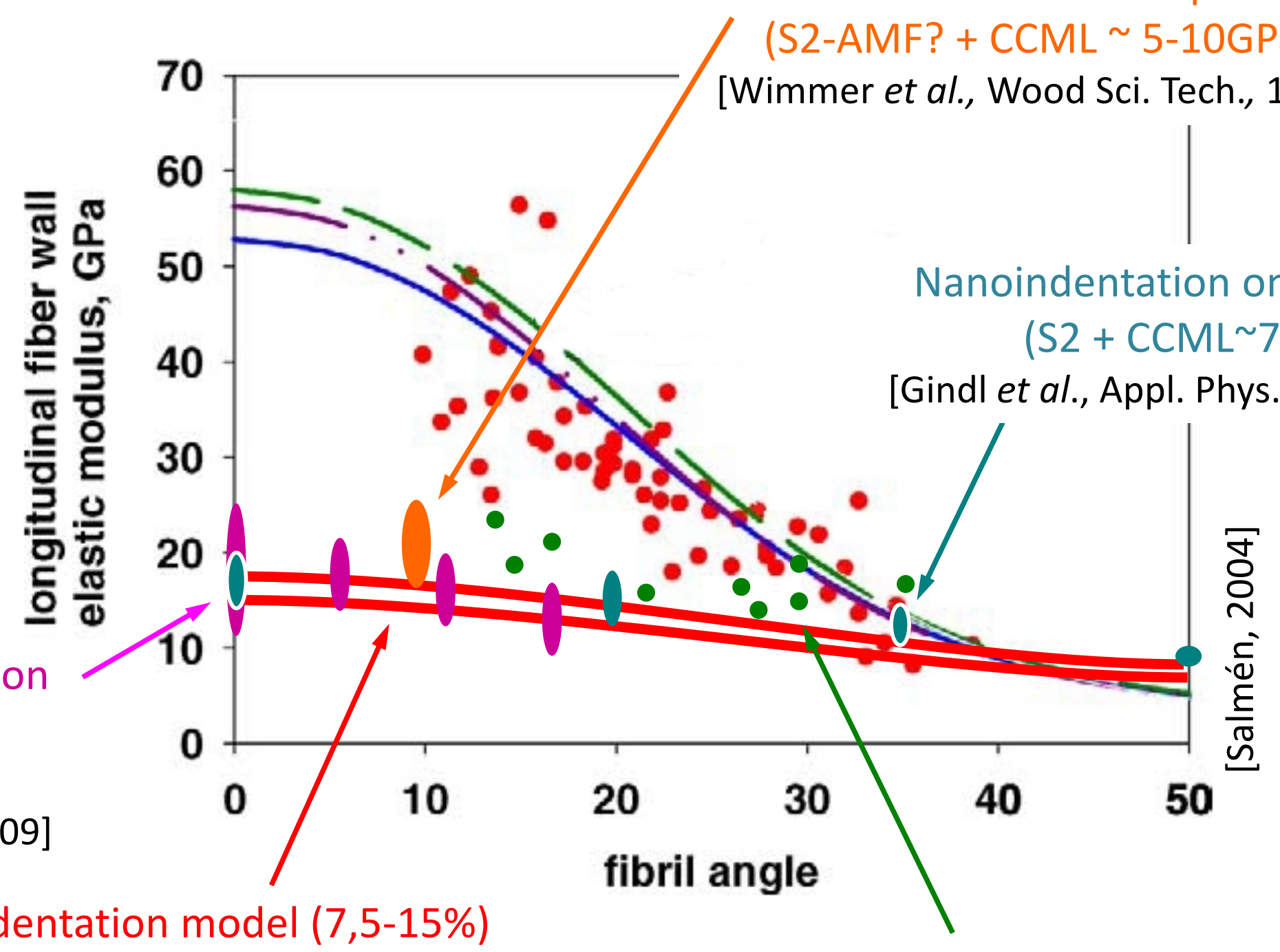

Anisotropic indentation model (7,5-15\%)

[Vlassak et al., J. Mech. Phys. Sol., 2003]

Nanoindentation on Pine (S2)

[Jäger et al., Comp. A, 2011]

[Tze et al., Comp. A, 2007]

[Eder, Arnould et al, Wood Sci. Technol., 2013] 


\section{Outline}

- Brief history and basic principles of scanning probe microscopy

- AFM principle and typical set-up

- Tip probe-sample surface interactions

- Tip and cantilever

- Deflection sensor and scanner

- Whole set-up

- Contact mechanics

- Operating modes (some)

- Contact

- Intermittent

- Force-distance curve and mechanical ,

- Image analysis and artefacts

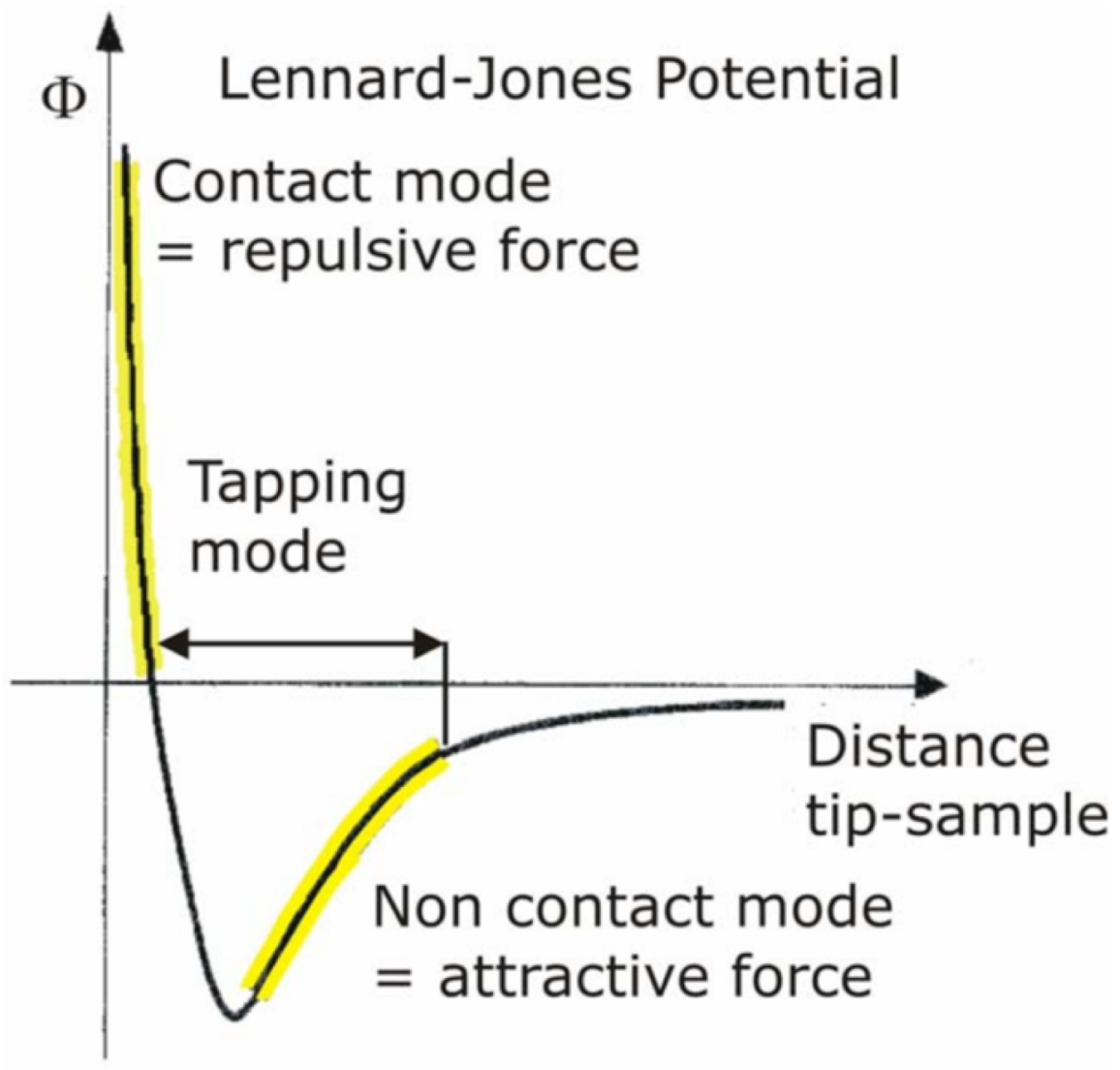

- Sample preparation 


\section{Force-distance curve}

- Van der Waals force, electrostatic force, contact stiffness, adhesion force, ...

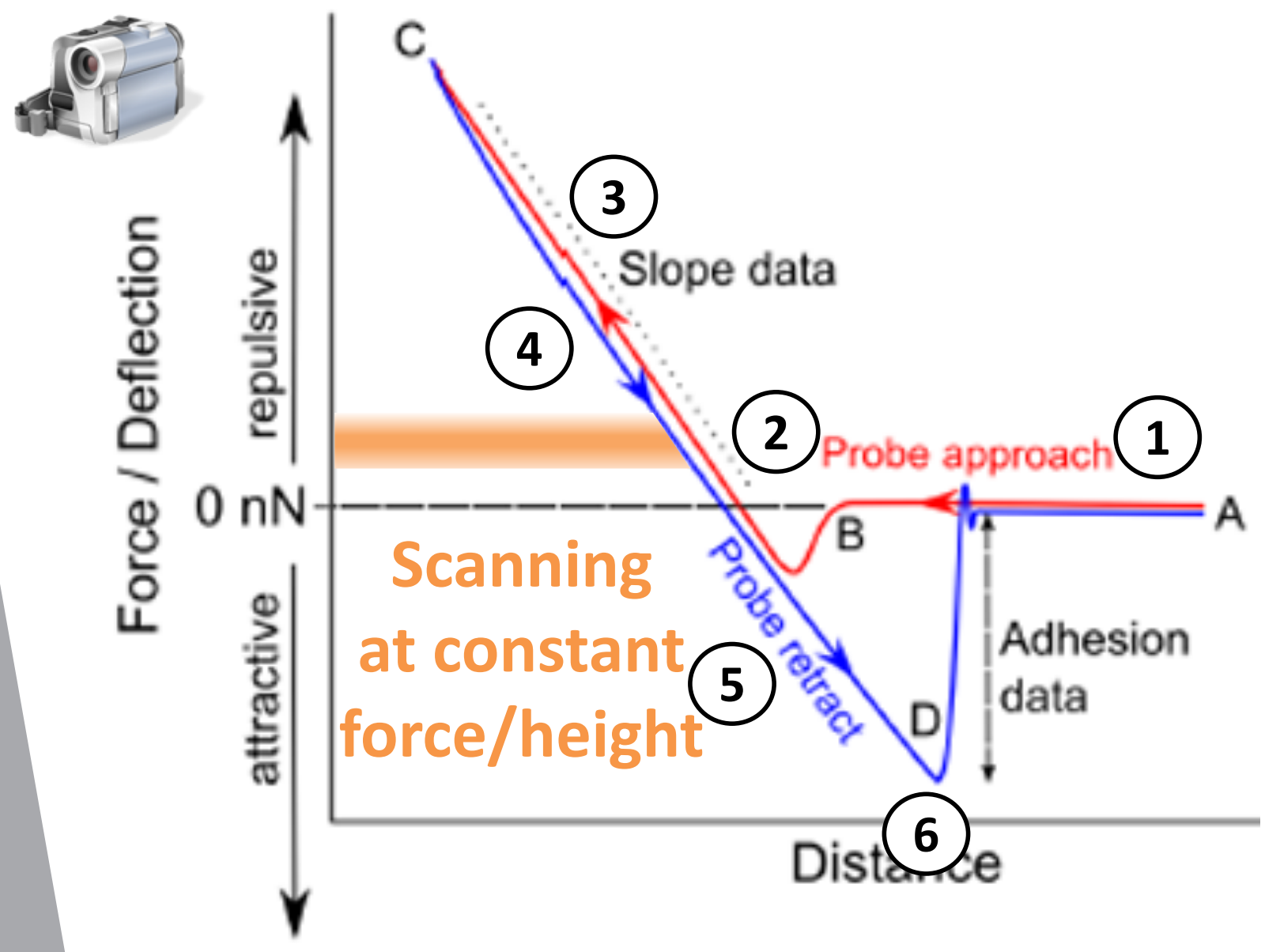

(1)

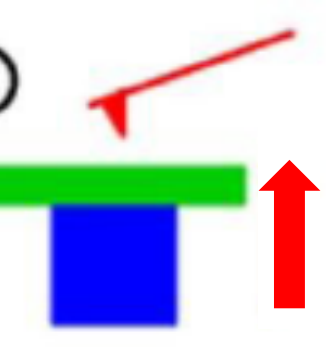

(2)

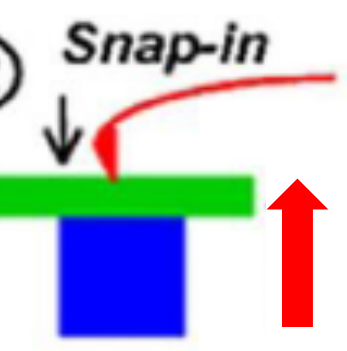

(3) Repulsion

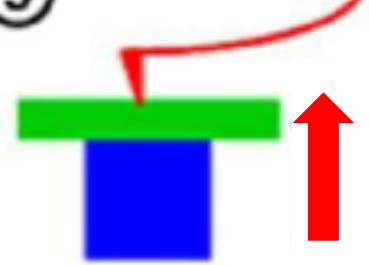

(4) Repulsion

4

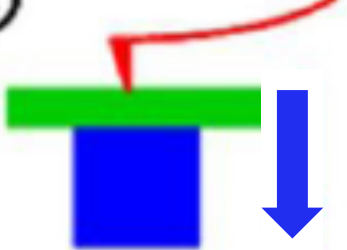

(5) Adhesion

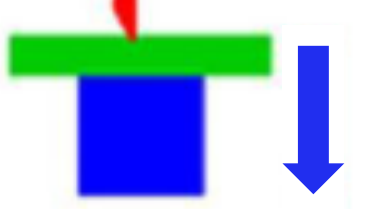

(6)

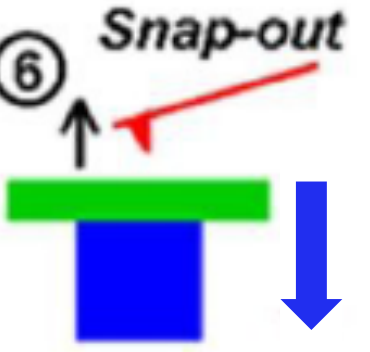

[B. Nysten, 2007] 


\section{Contact mode}

- Constant height or force and friction

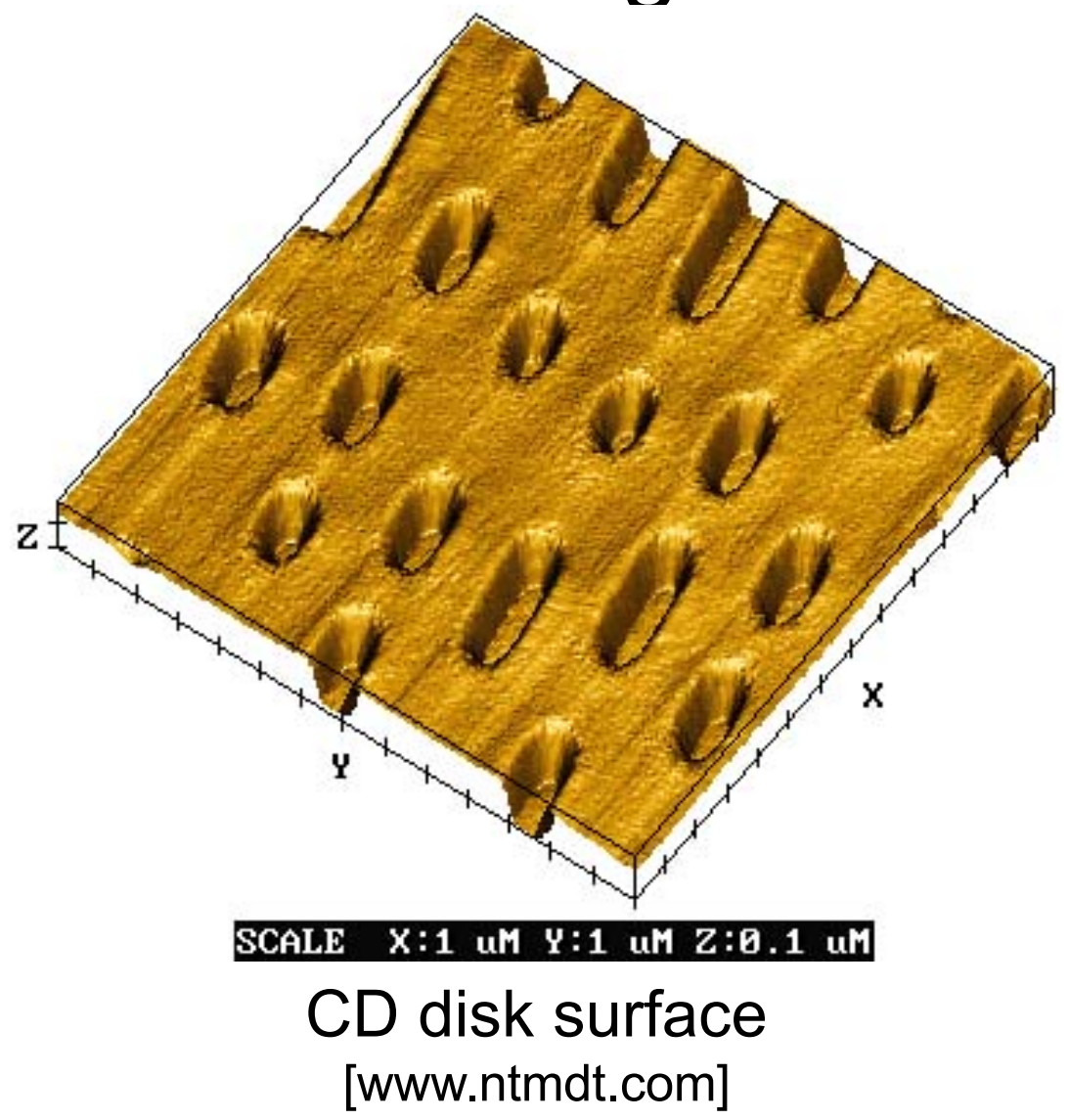

Cantilever with low stiffness $(0.01-1 \mathrm{~N} / \mathrm{m})$ \pm Fast scan speed in constant force mode (topography/closed-loop feedback) Limited topography in height force mode Tip wear, effect of sample elasticity Poor resolution (surface deformation, large tip surface contact area)

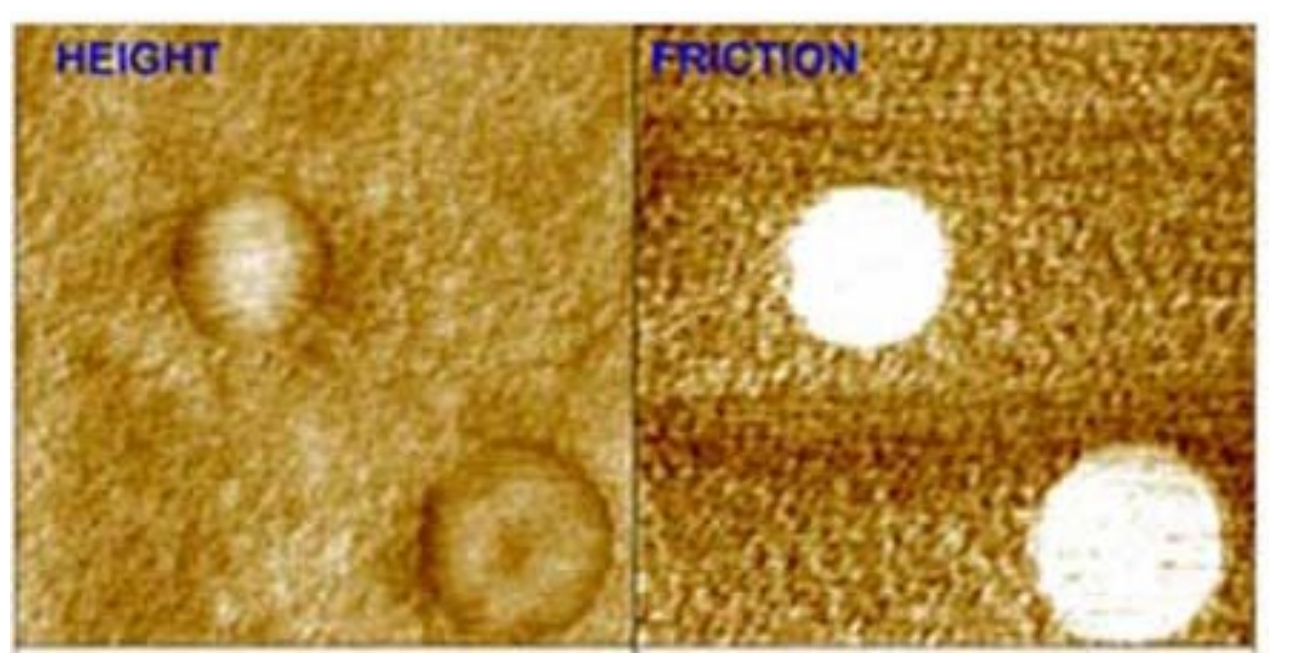

PS-PMMA polymer blend [Feldman et al., Langmuir, 1998] 


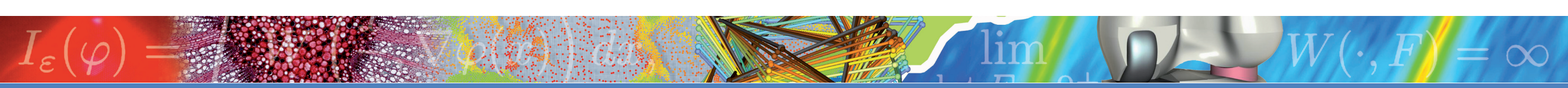

\section{Contact mode}

\section{- Force Modulation (FMM) DMA}

- Few nm oscillations @ few kHz, longitudinal or transversal!

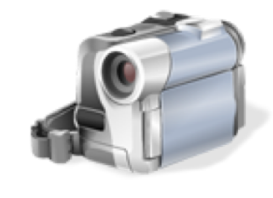

$\rightarrow$ Cantilever vibration amplitude and phase-shift related to surface elastic and viscoelastic properties

$\rightarrow$ Same limitations as F-d curves but better resolution ( $\approx 100 \mathrm{~nm}$ on polymer) and lower imaging time (scan rate $<0.5$ line/s)

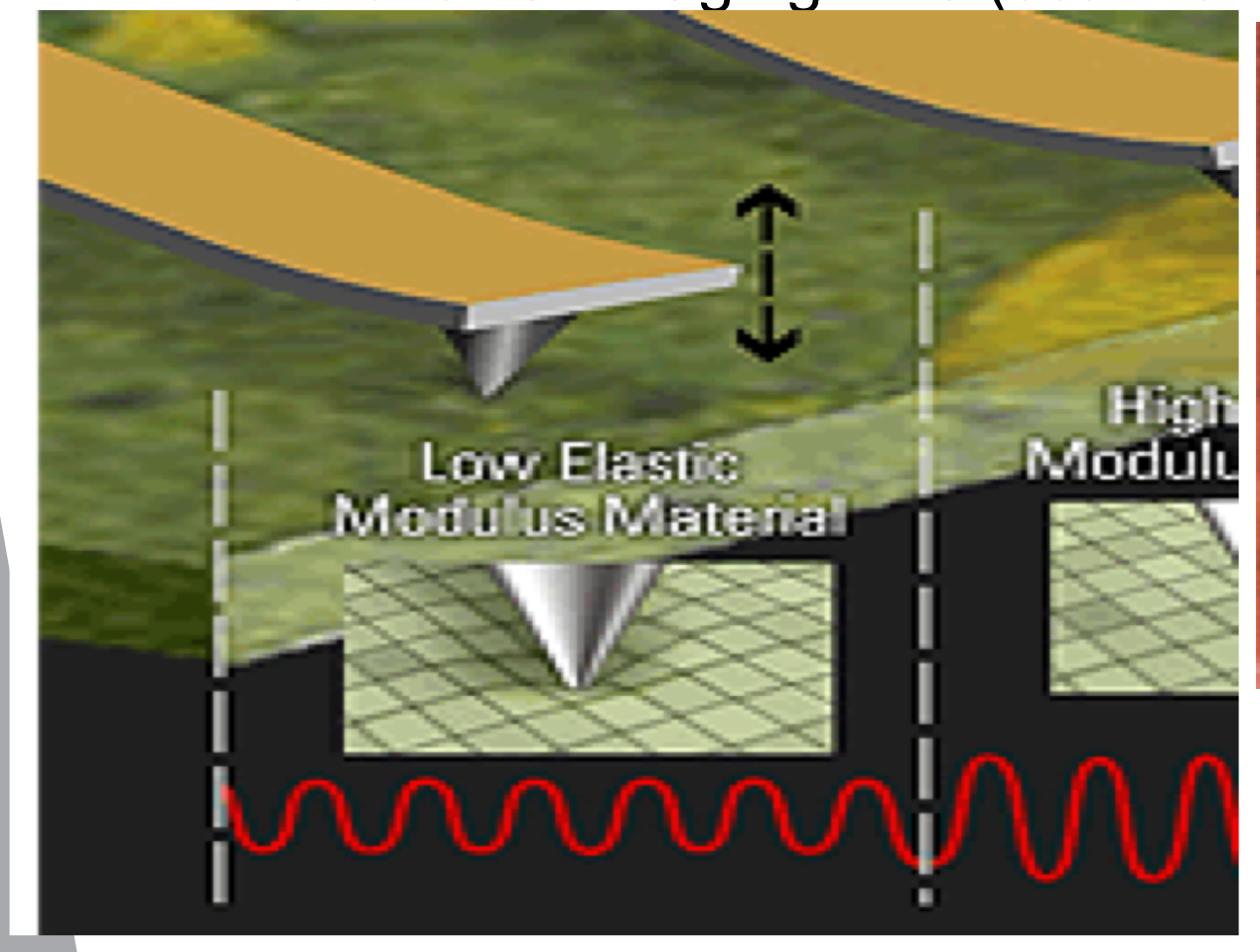

[www.afmuniversity.org]

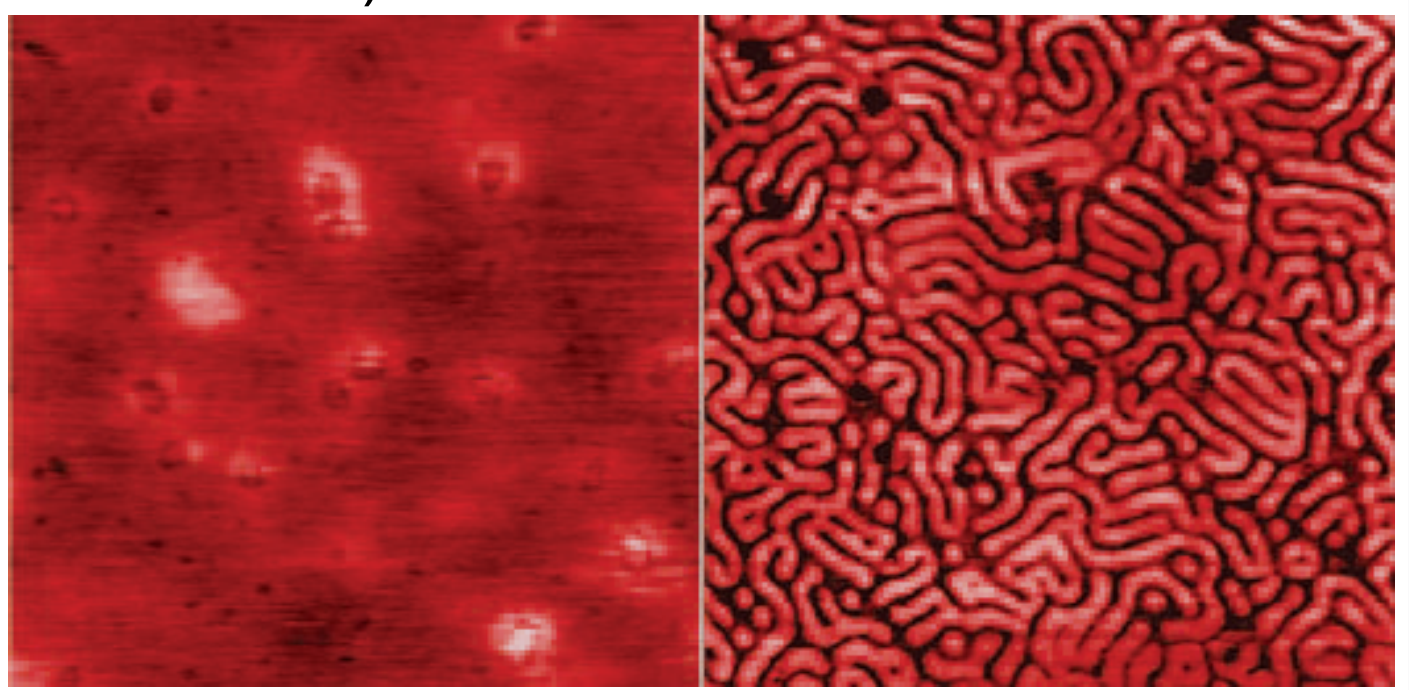

Topography and force modulation images (900 nm scans) of a two-phase block copolymer (softer $=$ black)

[www.veeco.com] 


\section{Contact Resonance-AFM (CR-AFM)}

IDEA: To probe the local elastic stiffness of the tip-sample system by means of cantilever's resonance frequency in contact mode at reduce applied force

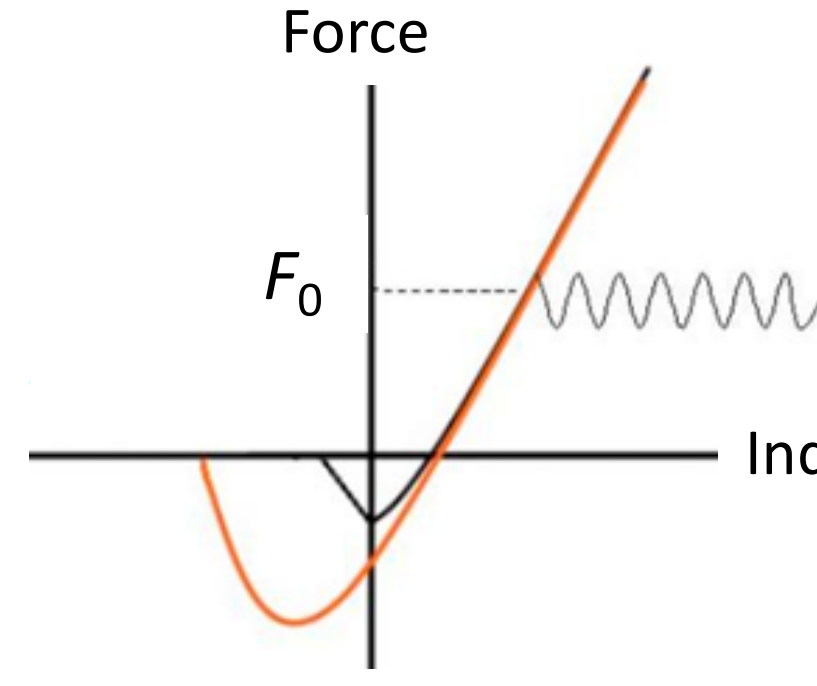

Cantilever-tip-sample system

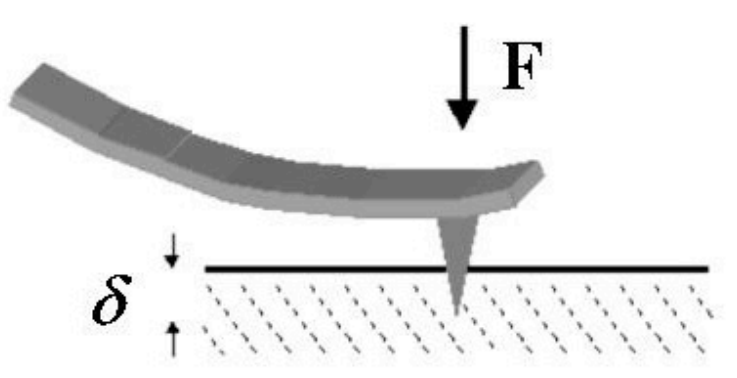

Applied force is modulated $F=F_{0}+F_{\text {excitation }} \times \sin \omega t$

if $F_{\text {excitation }}$ is small $\longrightarrow \approx$ LINEAR contact stiffness if $F_{0}$ is small $\longrightarrow$ Good spatial resolution + Hertz theory validity

$\begin{aligned} & \text { Point-mass model } \\ & \text { [Rabe et al, 1996] }\end{aligned} f_{0}=\frac{1}{2 \pi} \sqrt{\frac{k_{c}+k_{N}}{m^{*}}}$ with $m^{*}=\frac{1}{4} m+m_{\text {tip }}$

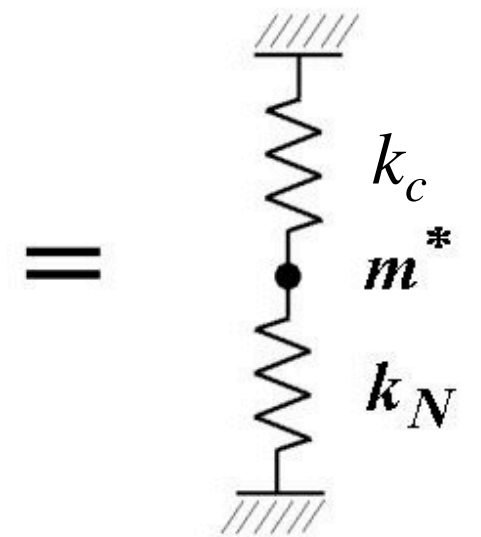

Amplitude of vibration and resonance frequency depend on the contact stiffness $k_{\mathrm{N}} \ldots$ but how? 


\section{Cantilever dynamic and response}

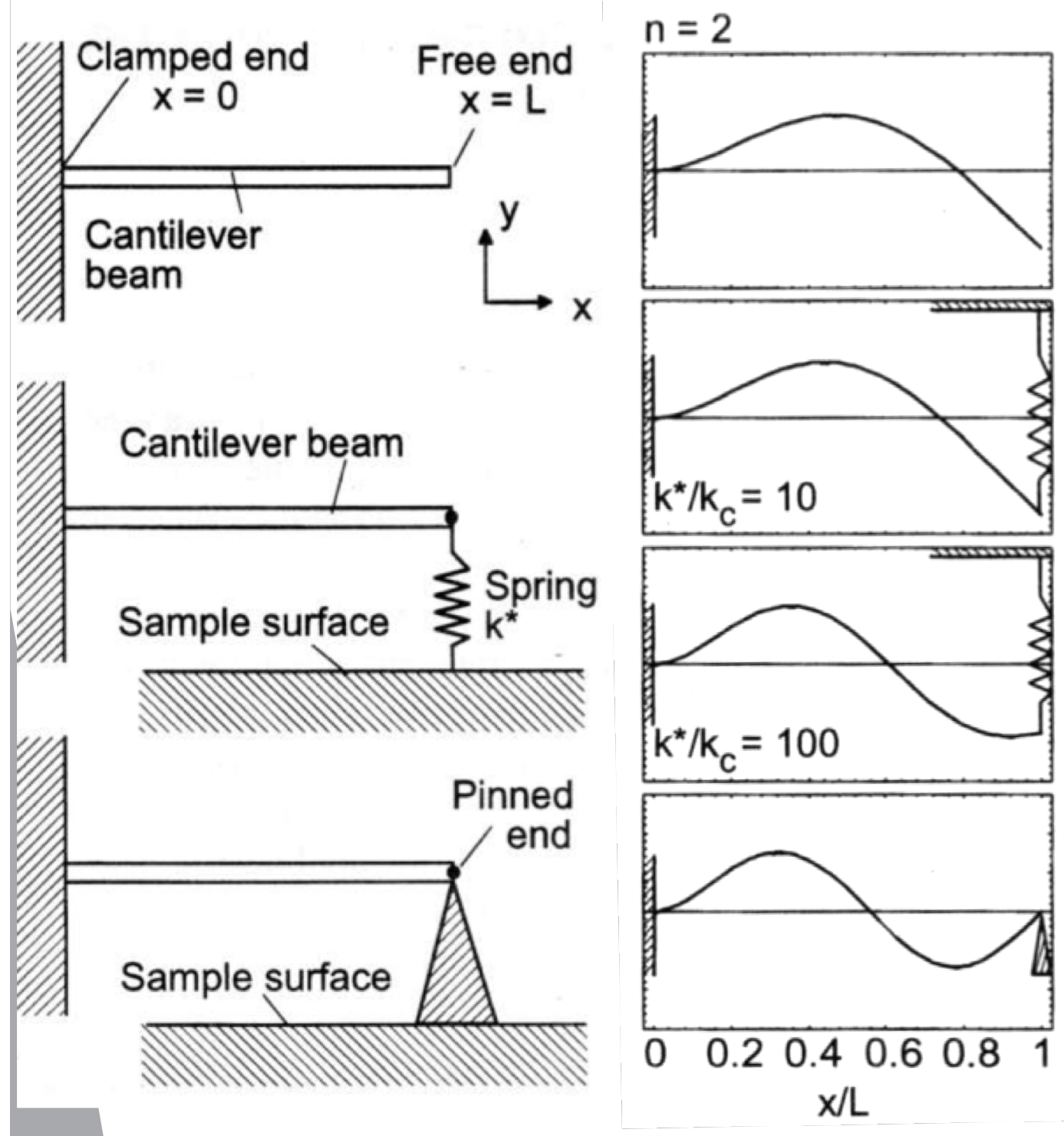

[Rabe et al, 1996]

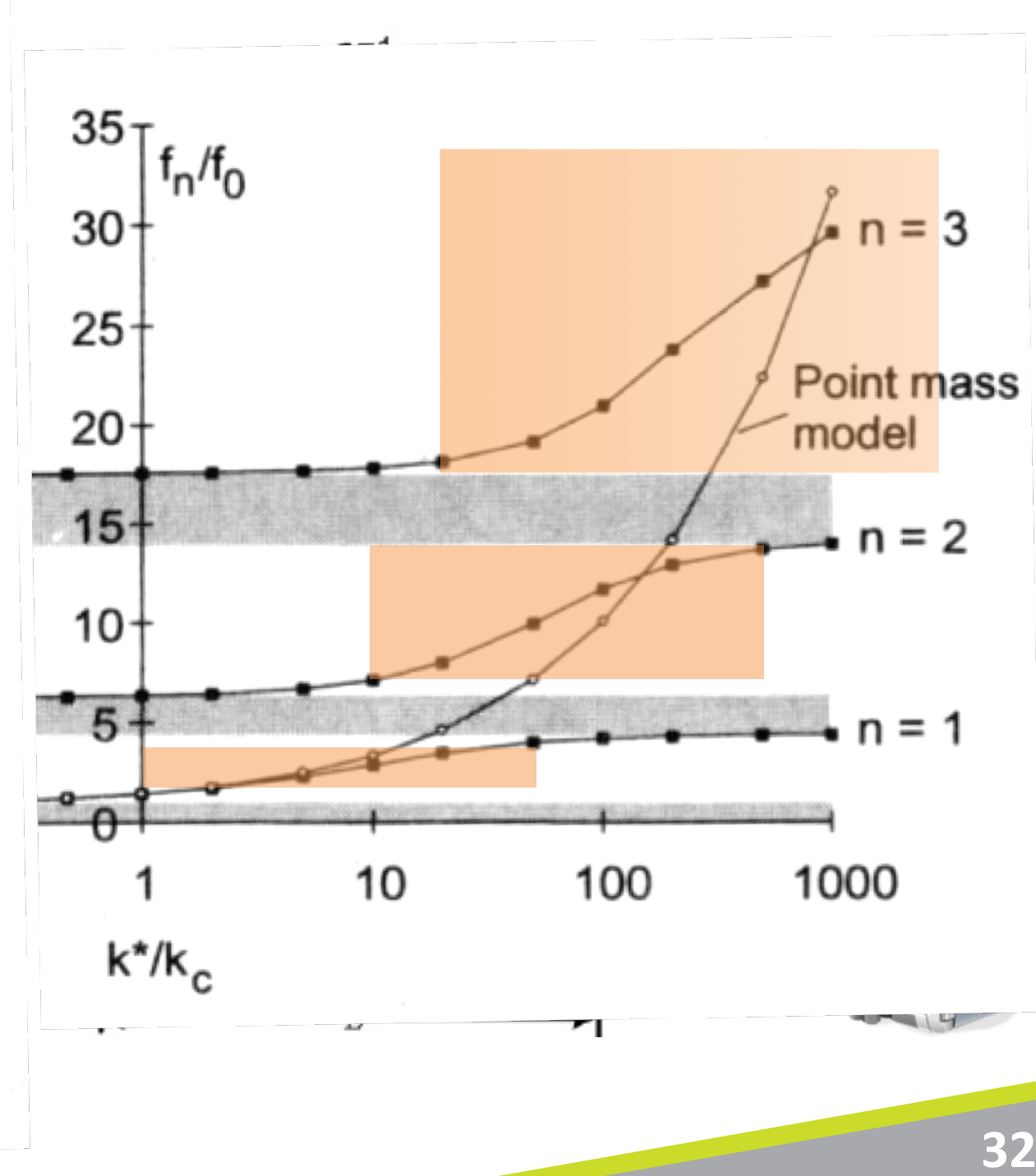


Contact Resonance-AFM principle [Arinero and Lévêque, Rev. Phys. Instr., 2003] with Dual Resonance Frequency Tracking

[Gannepalli et al., Nanotechnol. 2011]

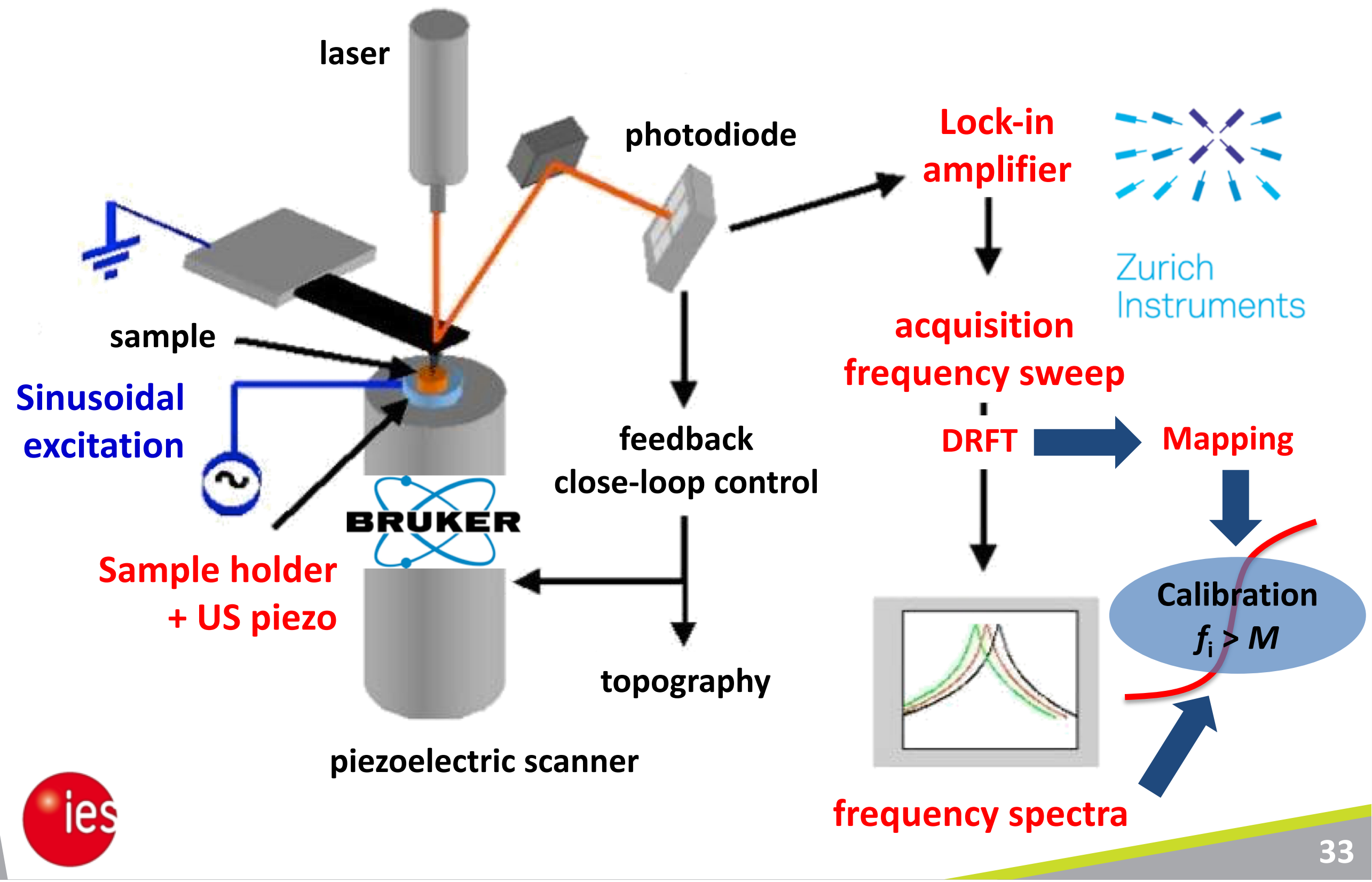


$I_{\varepsilon}(\varphi)=8 x_{2}$ Chestnut tension wood

[Arnould and Arinero, Comp. A, 2015, 74: 79-76]

Effect of the topography and sample orientation [Konnerth et al, J. Mater. Sci., 2009]

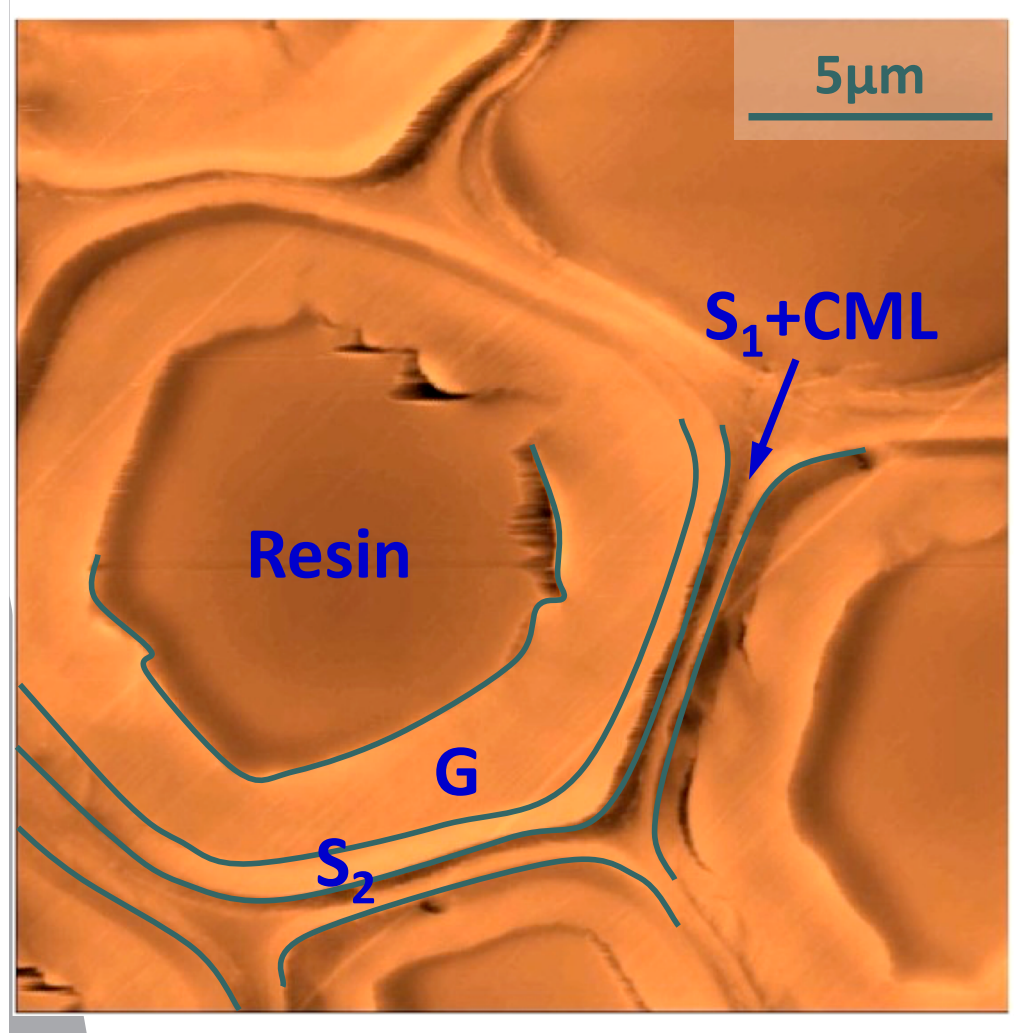

Topography (nm)
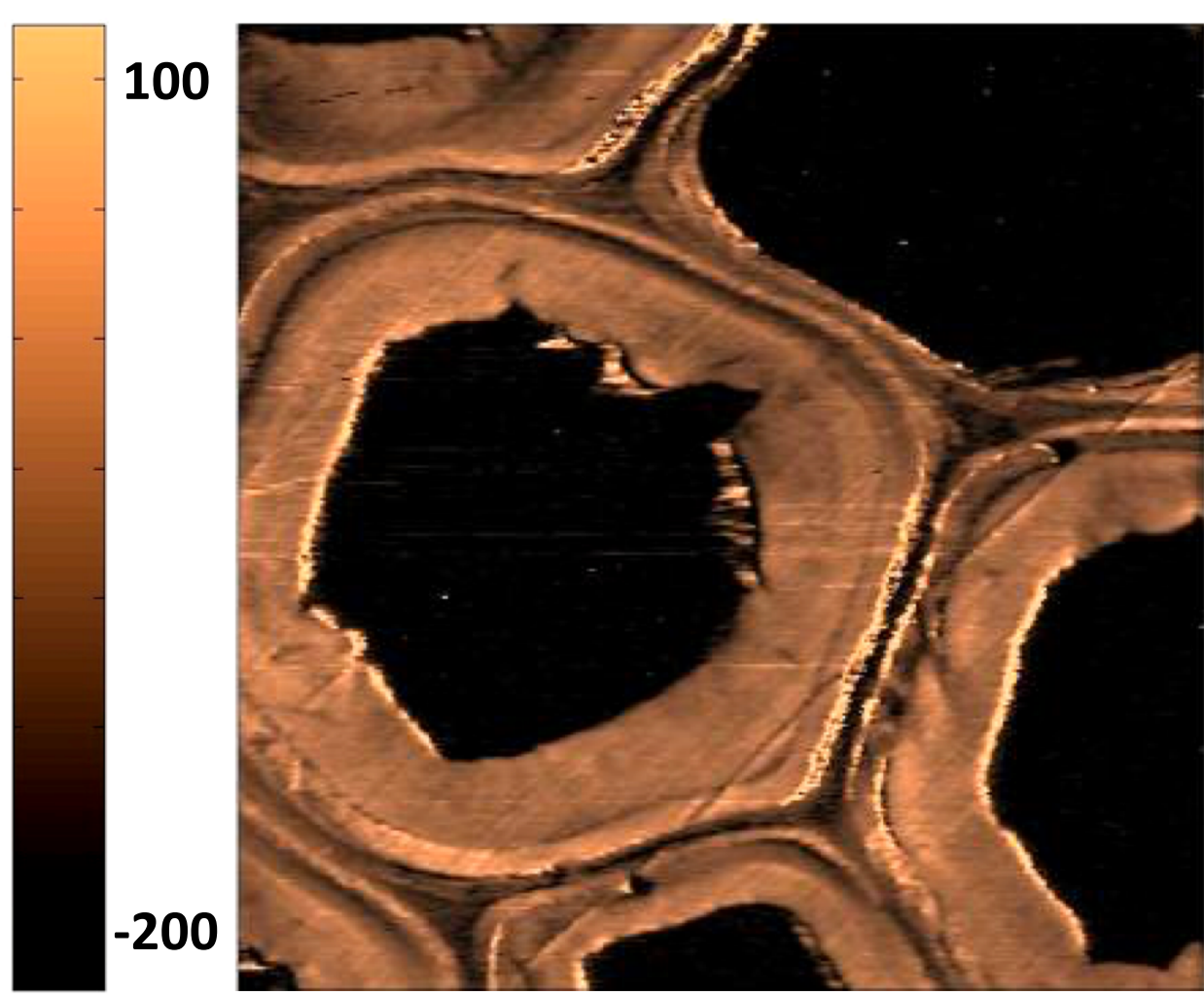

Contact modulus (GPa)

Veeco Enviroscope, CR-AFM, Nanoworld ARROW FMR, 2,8 N/m, 55 nm (adh), $180 \mathrm{nN}$ 


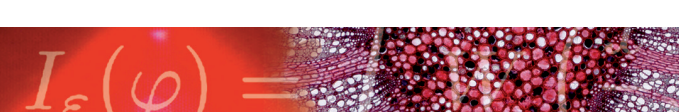

\section{Chestnut tension wood}

[Arnould and Arinero, Comp. A, 2015]

Effect of sample orientation [Konnerth et al, J. Mater. Sci., 2009]
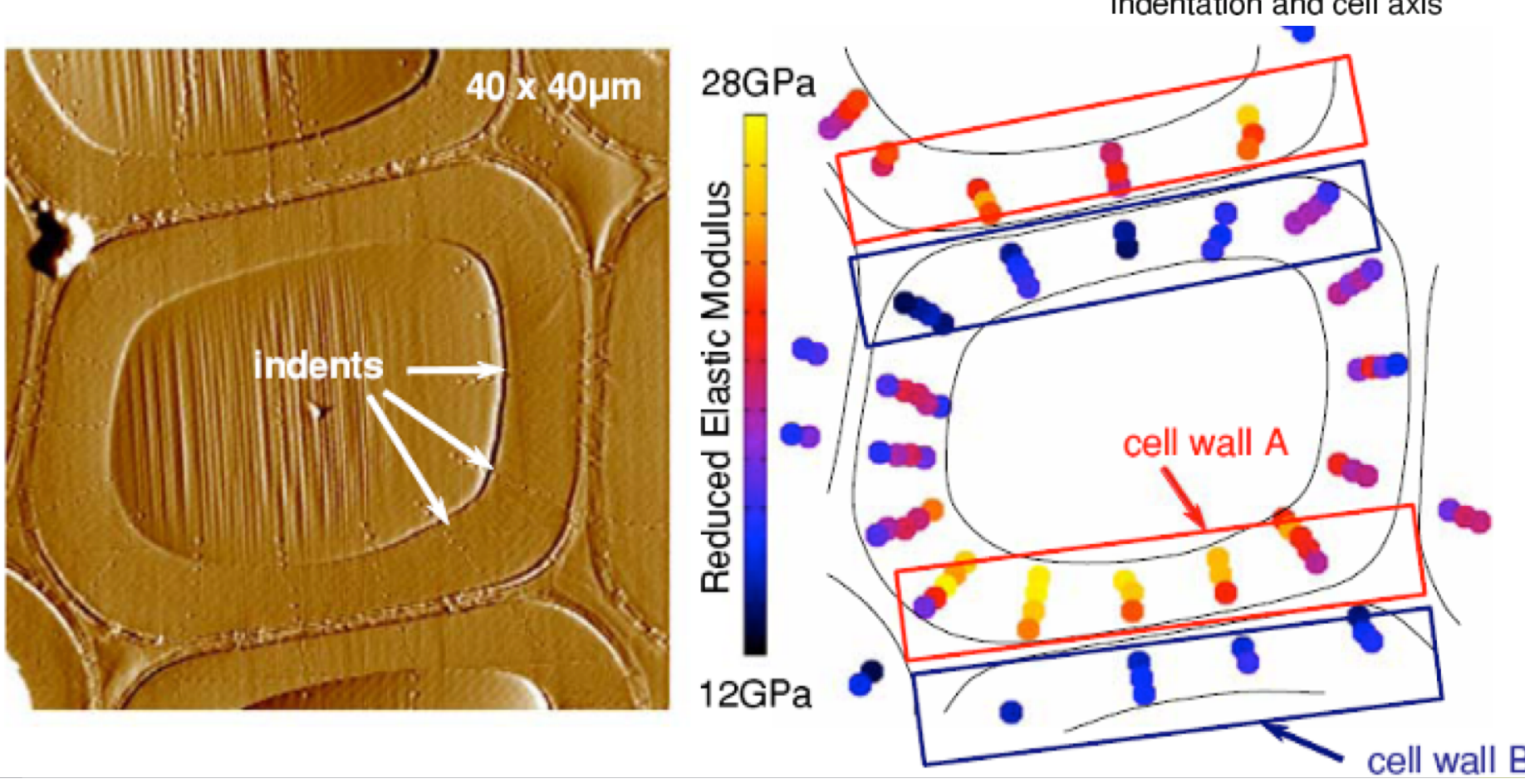


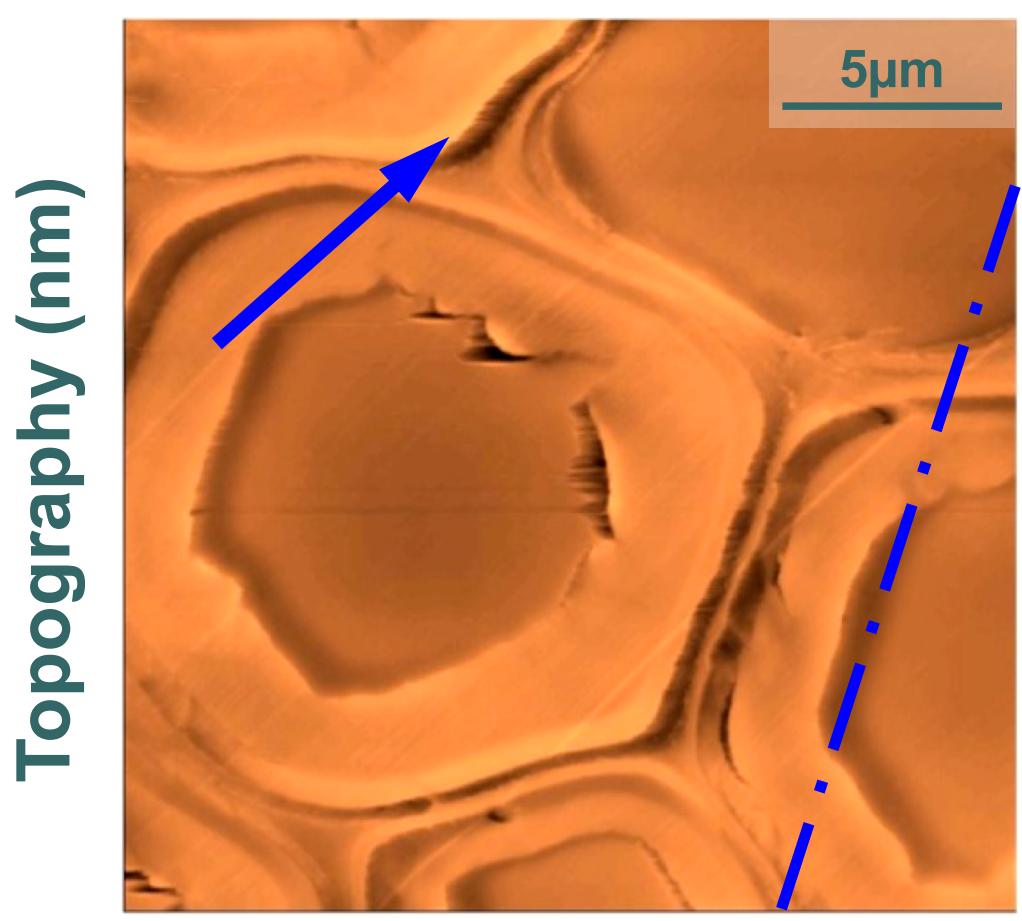

Simplified model

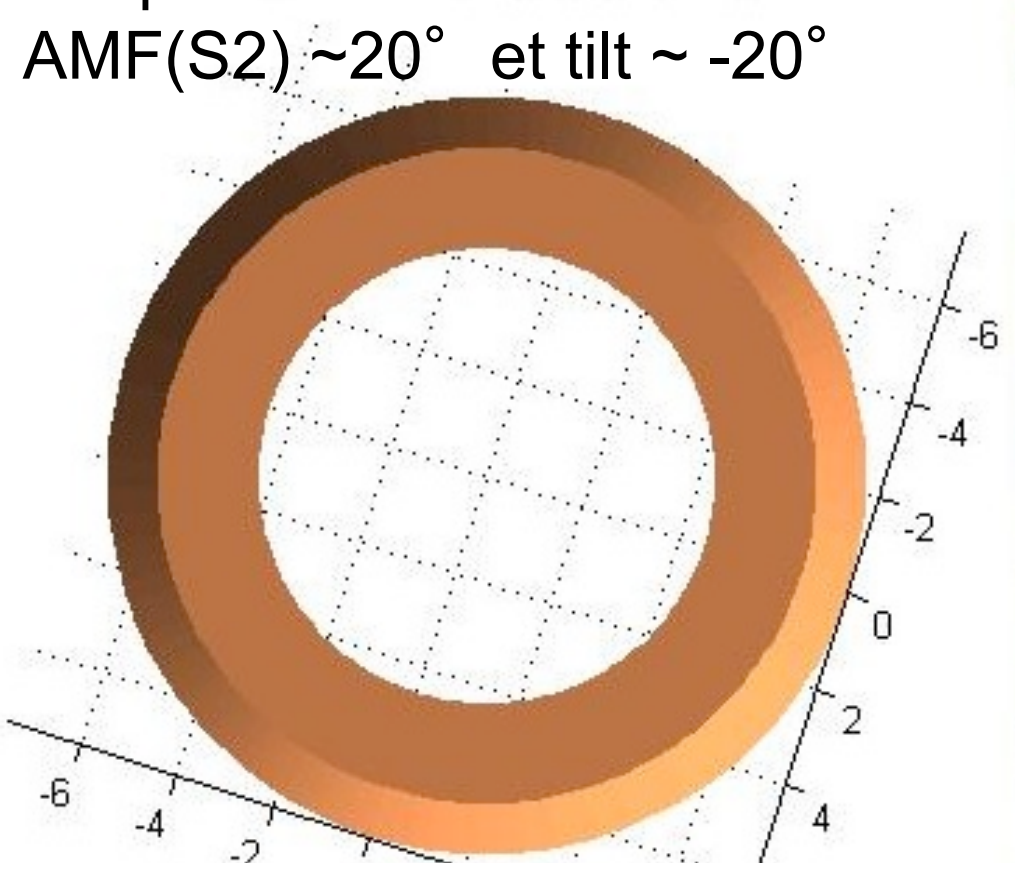

Cutting angle / MFA $\alpha\left({ }^{\circ}\right)$
100

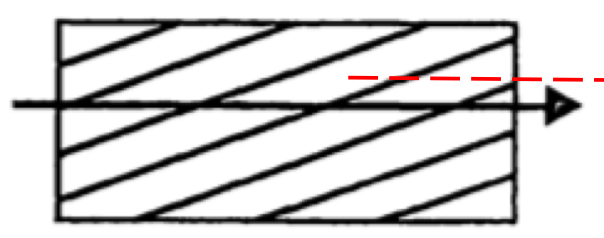

R.H.S. S2

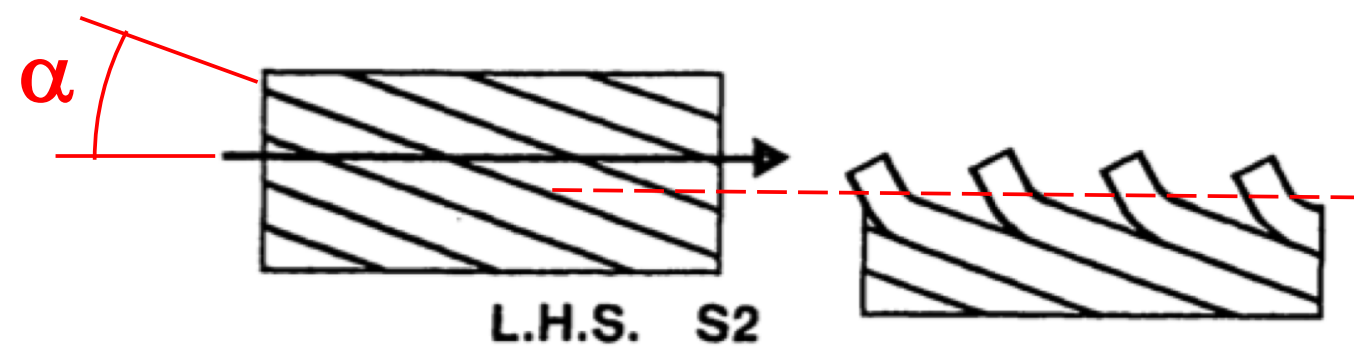

[Shaune et al, Holzforschung, 1994]
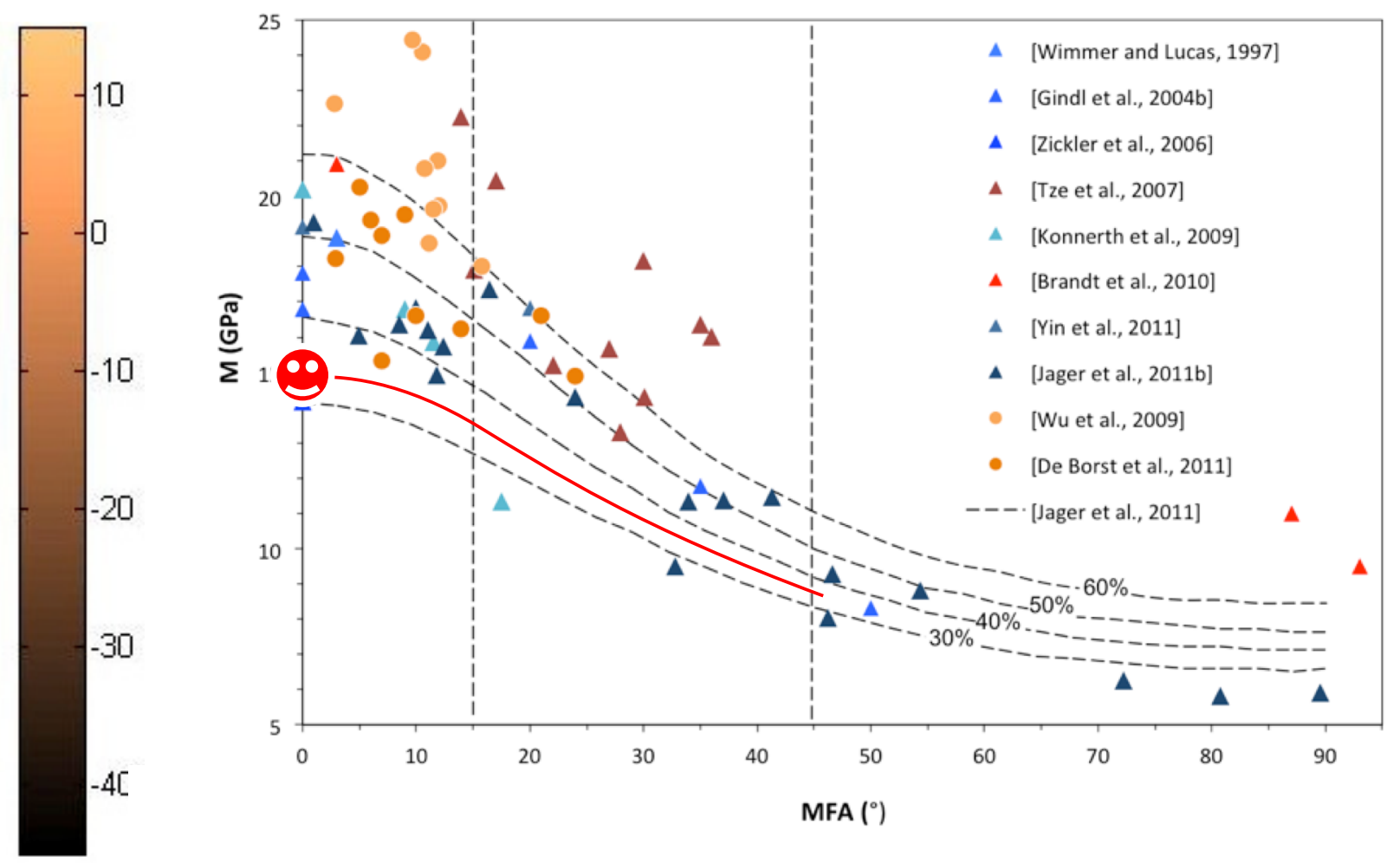

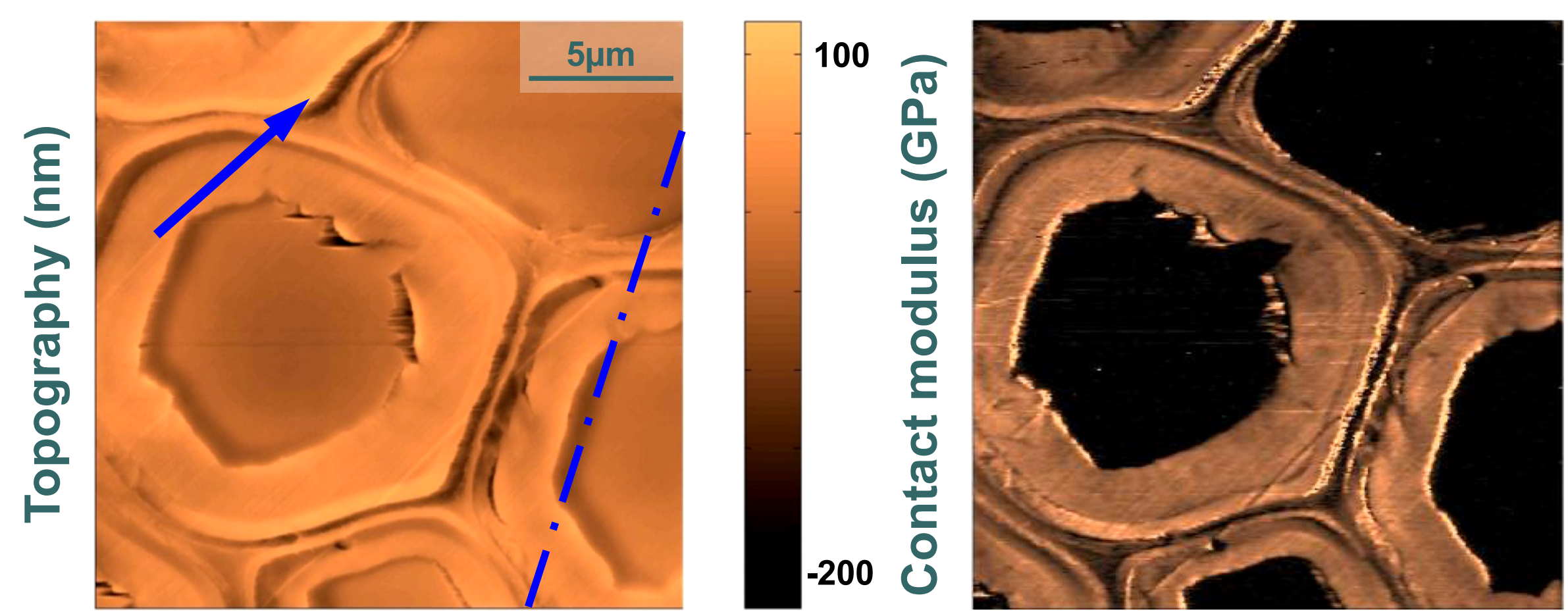

Simplified model

$\operatorname{AMF}(\mathrm{S} 2) \sim 20^{\circ}$ et tilt $\sim-20^{\circ}$

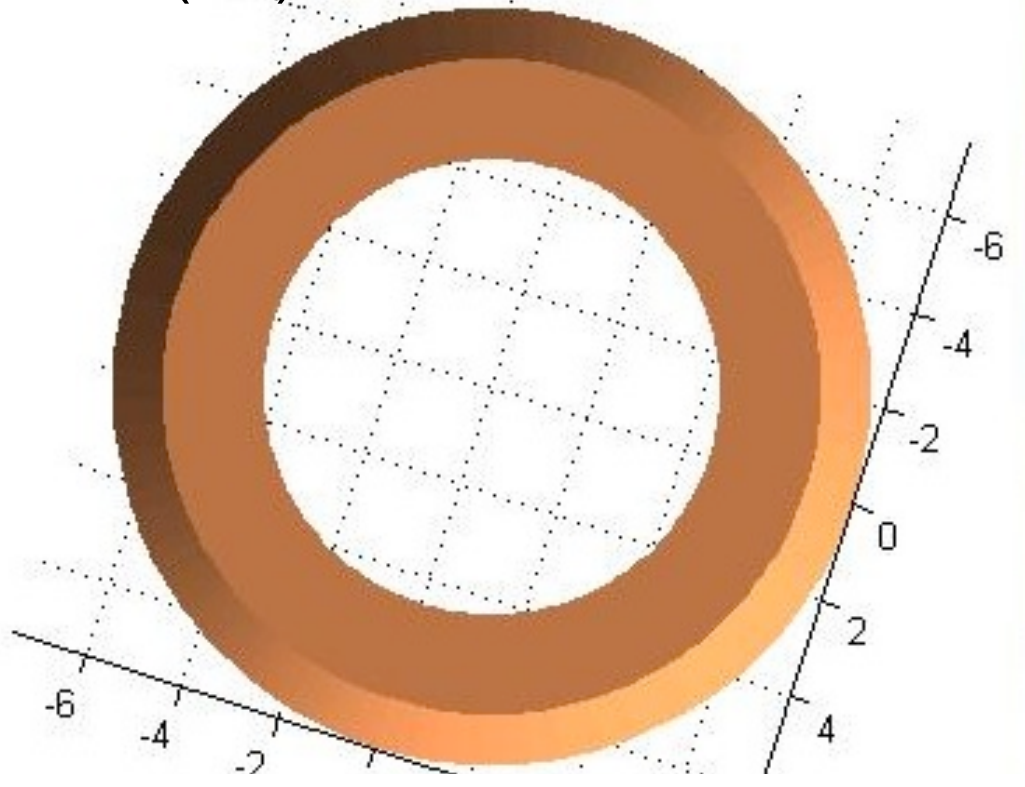

Cutting angle / MFA $\alpha\left({ }^{\circ}\right)$

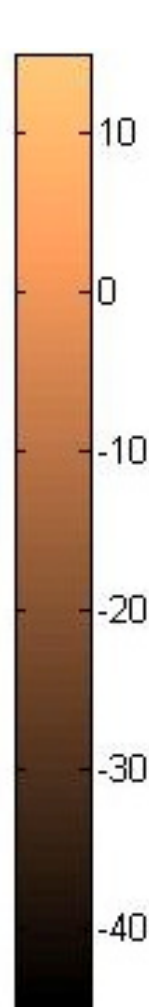

40

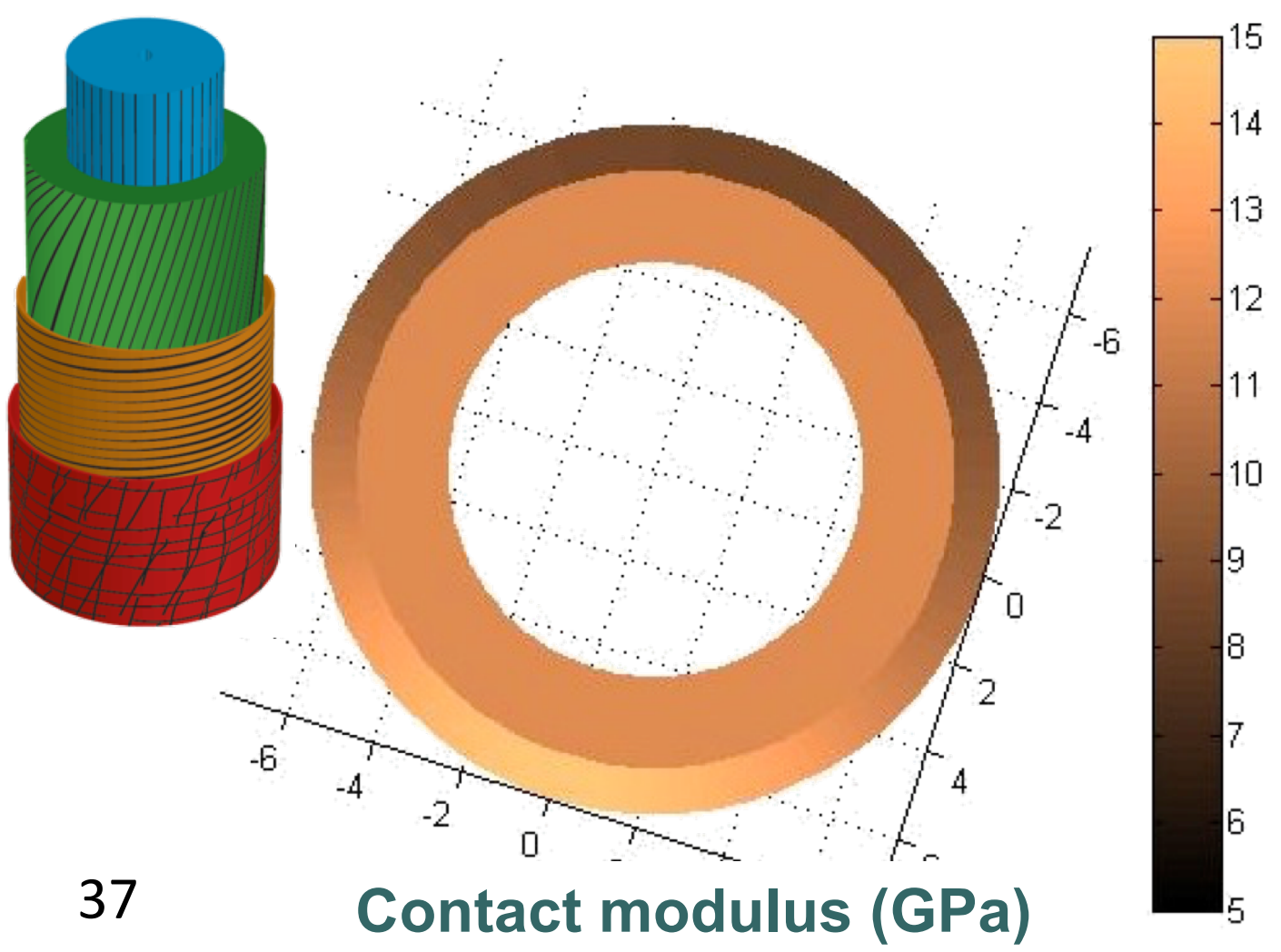




\section{Contact mode}

- Abrasion

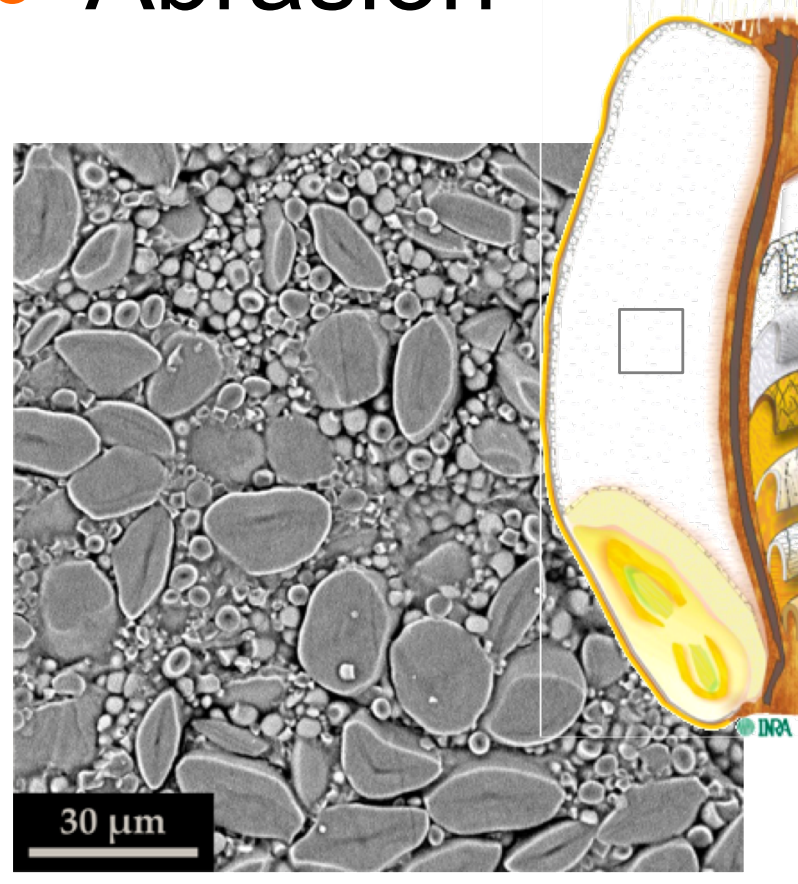

Starch granules inside a wheat endosperm (SEM)

Occurrence of "lubricated" interface in soft wheat

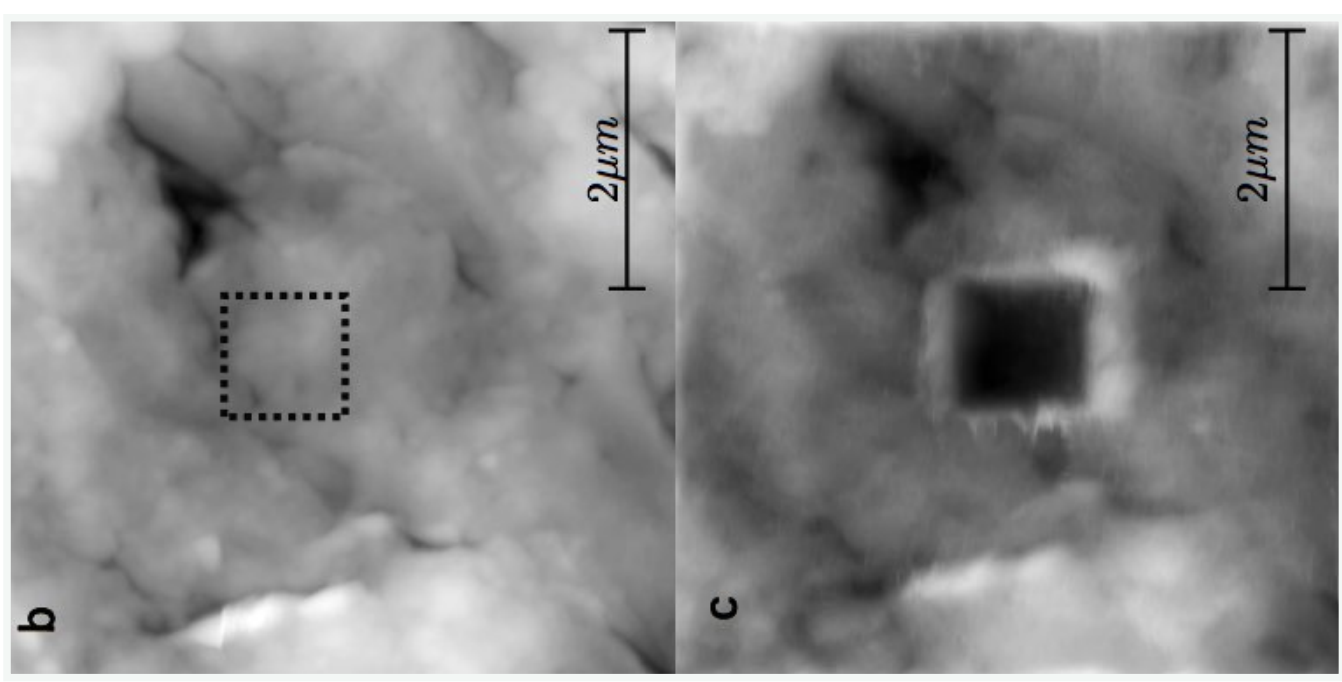

Tablet of pure gluten before/after abrasion (SEM)

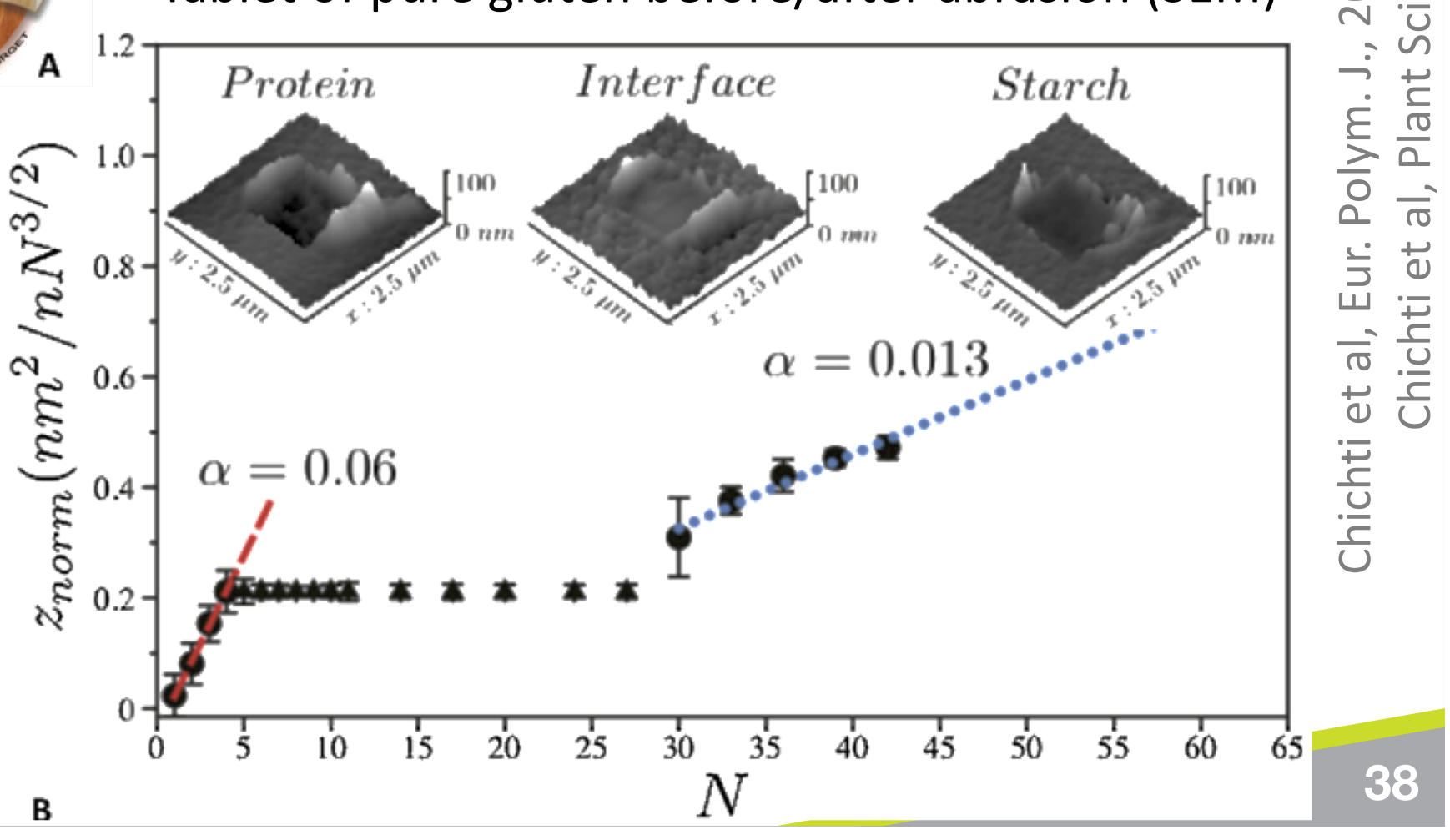




\section{Contact mode}

\section{- Scanning Thermal Microscopy (SThM)}

[Konnerth et al., Holzforschung, 2008]

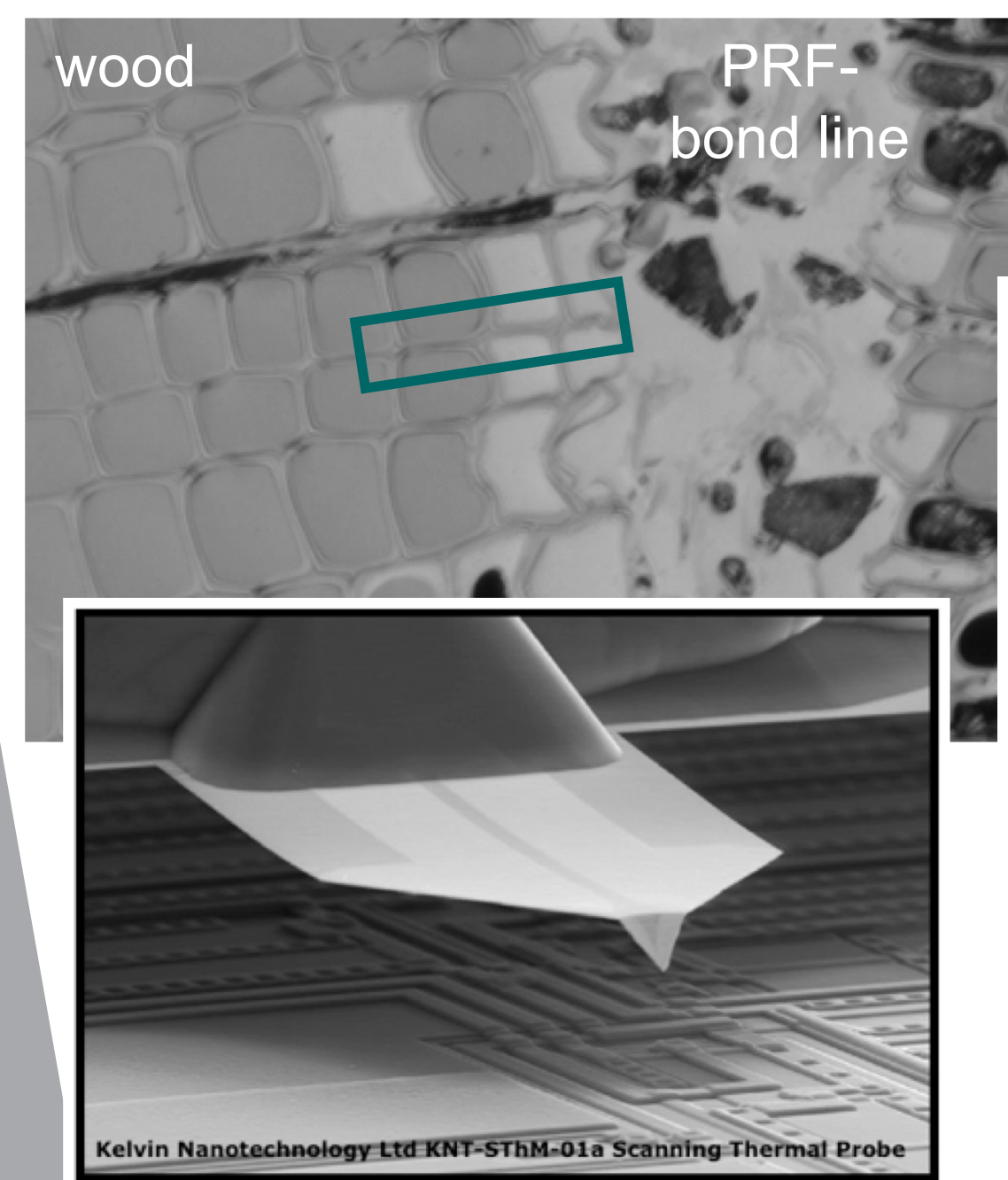

$$
R \approx 100 \mathrm{~nm}
$$

Scan rate $0.1-0.5 \mathrm{~Hz}$ Spruce wood

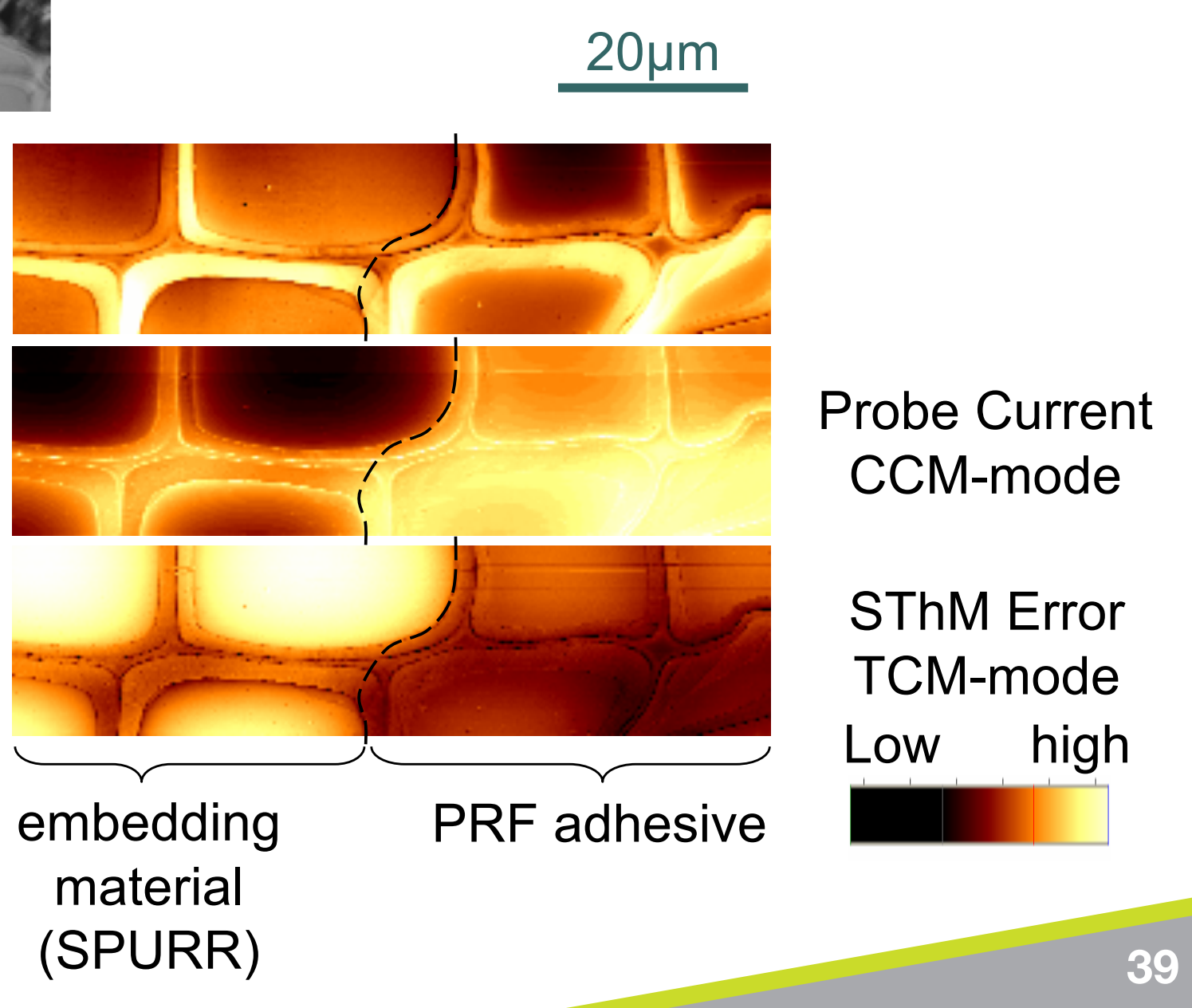




\section{Force-distance curve}

- Van der Waals force, electrostatic force, contact stiffness, adhesion force, ...

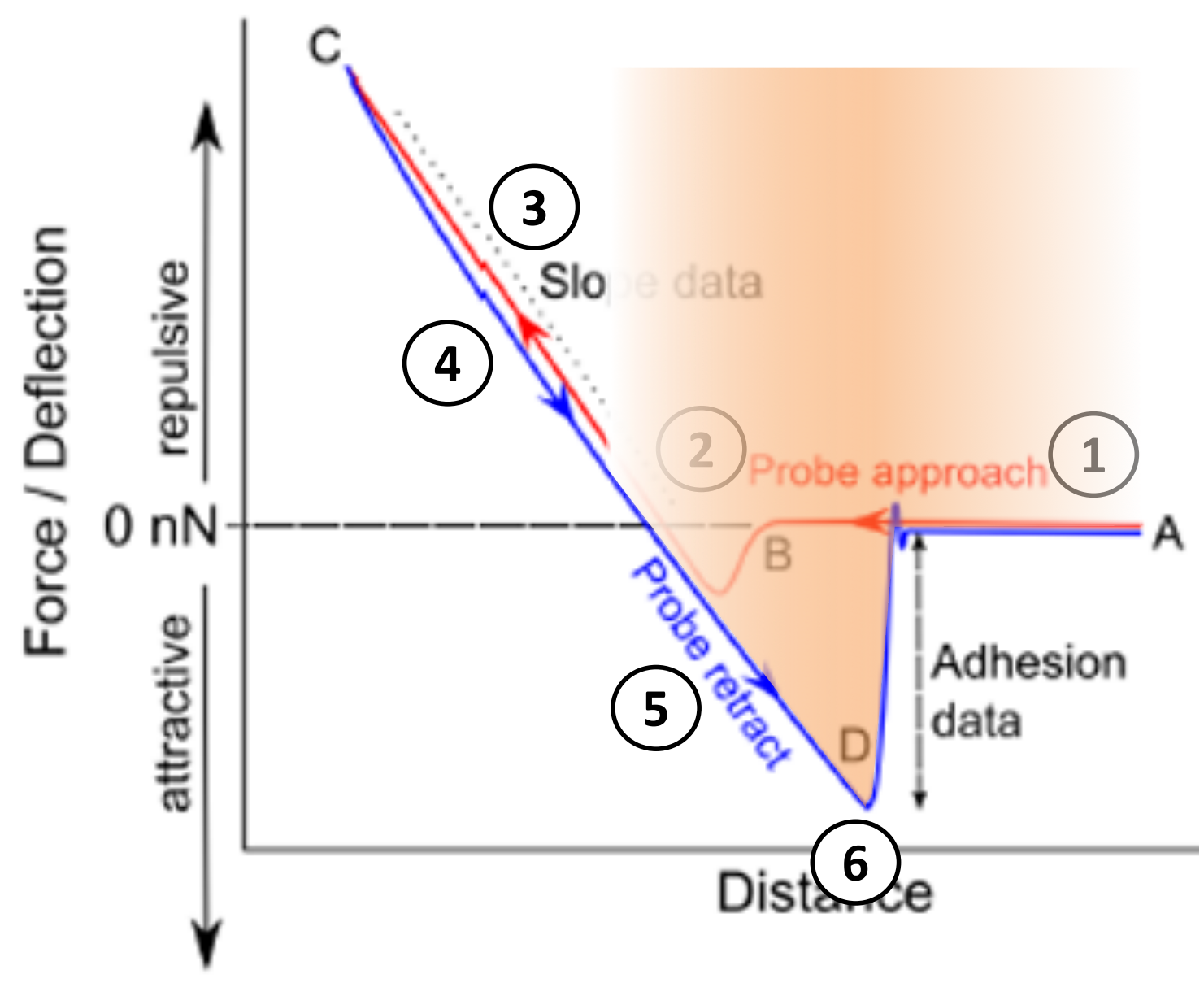

(1)

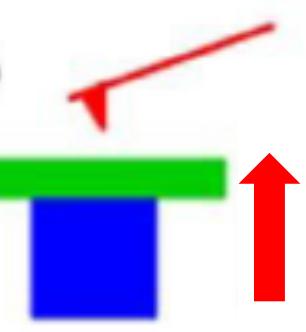

(2)

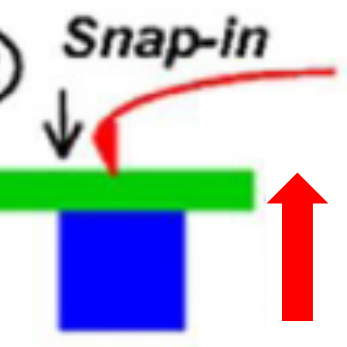

(3) Repulsion

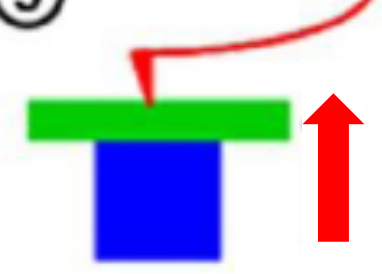

(4) Repulsion

(4)

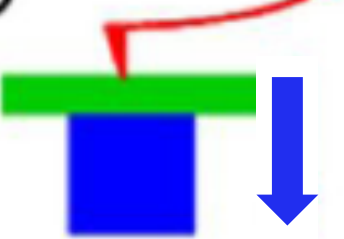

(5) Adhesion

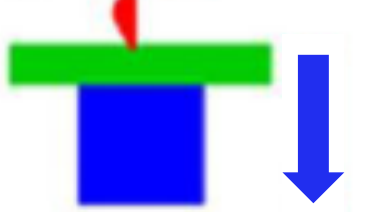

(6)

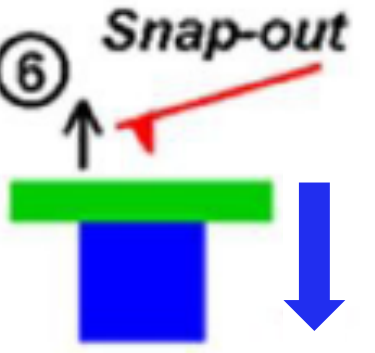

[B. Nysten, 2007] 


\section{Intermittent contact/tapping ${ }^{\mathrm{TM}} /$...}

\section{- Knocking on the surface!}

$\rightarrow$ Linear harmonic oscillator approximation (free)
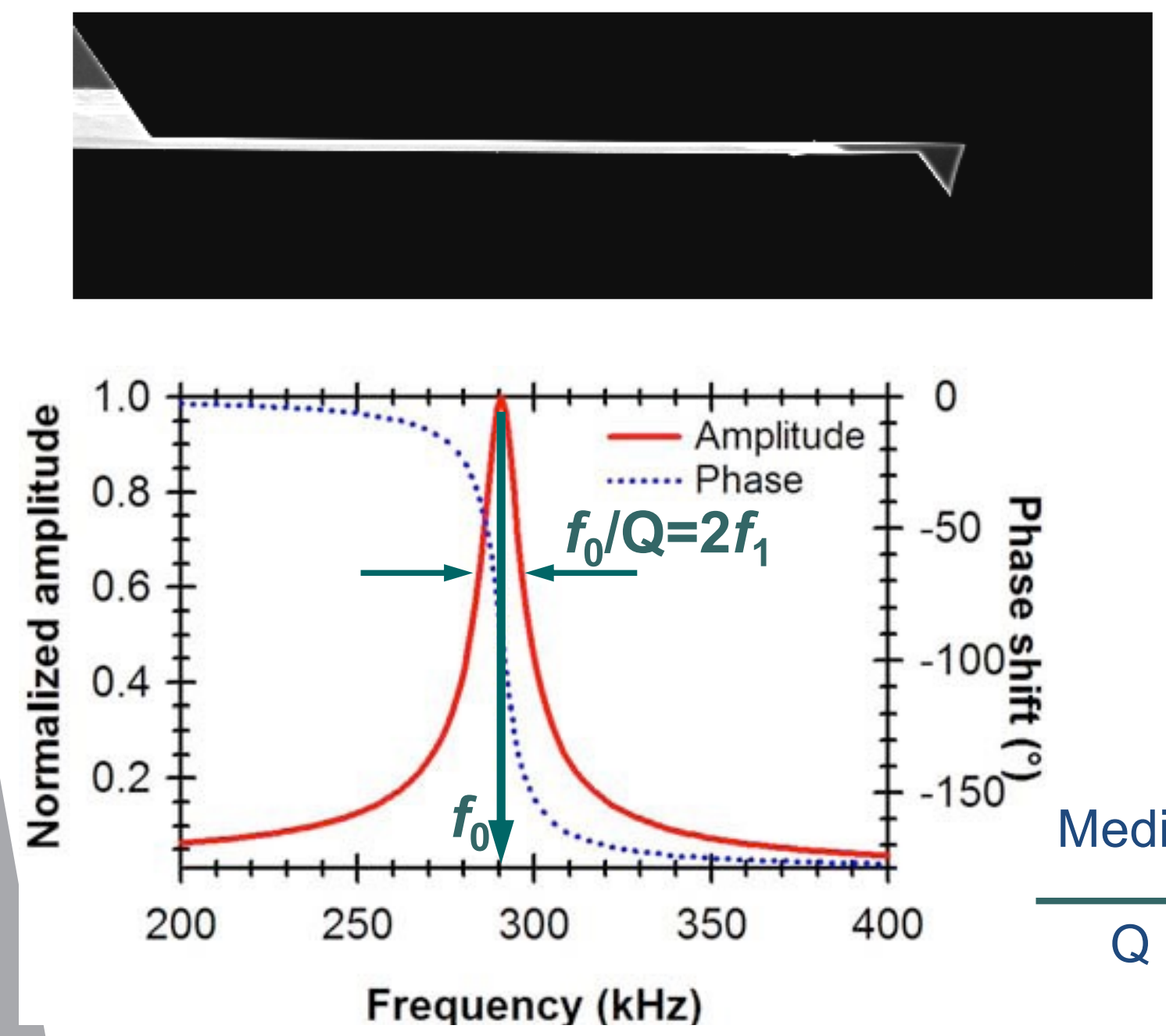

$$
\omega_{0}=\sqrt{\frac{k_{c}}{m_{\text {eff }}}}
$$

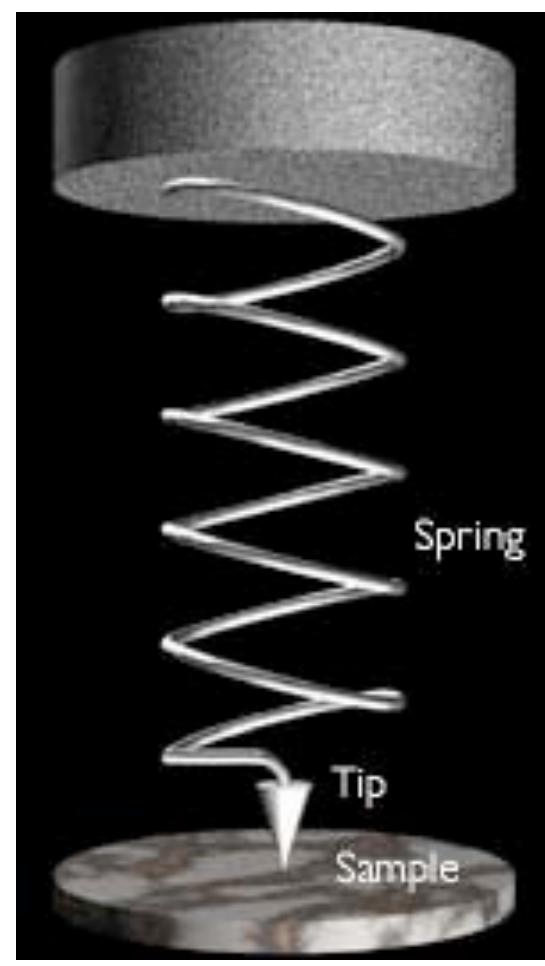

$k_{C}>10 \mathrm{~N} / \mathrm{m}$

$f_{0}>100 \mathrm{kHz}$

Sample

\begin{tabular}{c|c|c|c} 
Media & Liquid & Air & $\begin{array}{c}\text { High } \\
\text { vacuum }\end{array}$ \\
\hline Q & 10 & 400 & $10^{4}$
\end{tabular}


OScan at constant vibration amplitude $=$ topography + stiffness contrast

$\rightarrow$ soft (topography) $A_{s} / A_{0}>0.8+A_{0} \downarrow /$ hard (elasticity) $A_{s} / A_{0}<0.4+A_{s} \uparrow$ tapping

$\partial$ Linear harmonic oscillator approximation in a force gradient

[Magonov et al., Surf. Sci. Lett., 1997; Nysten, 2007]
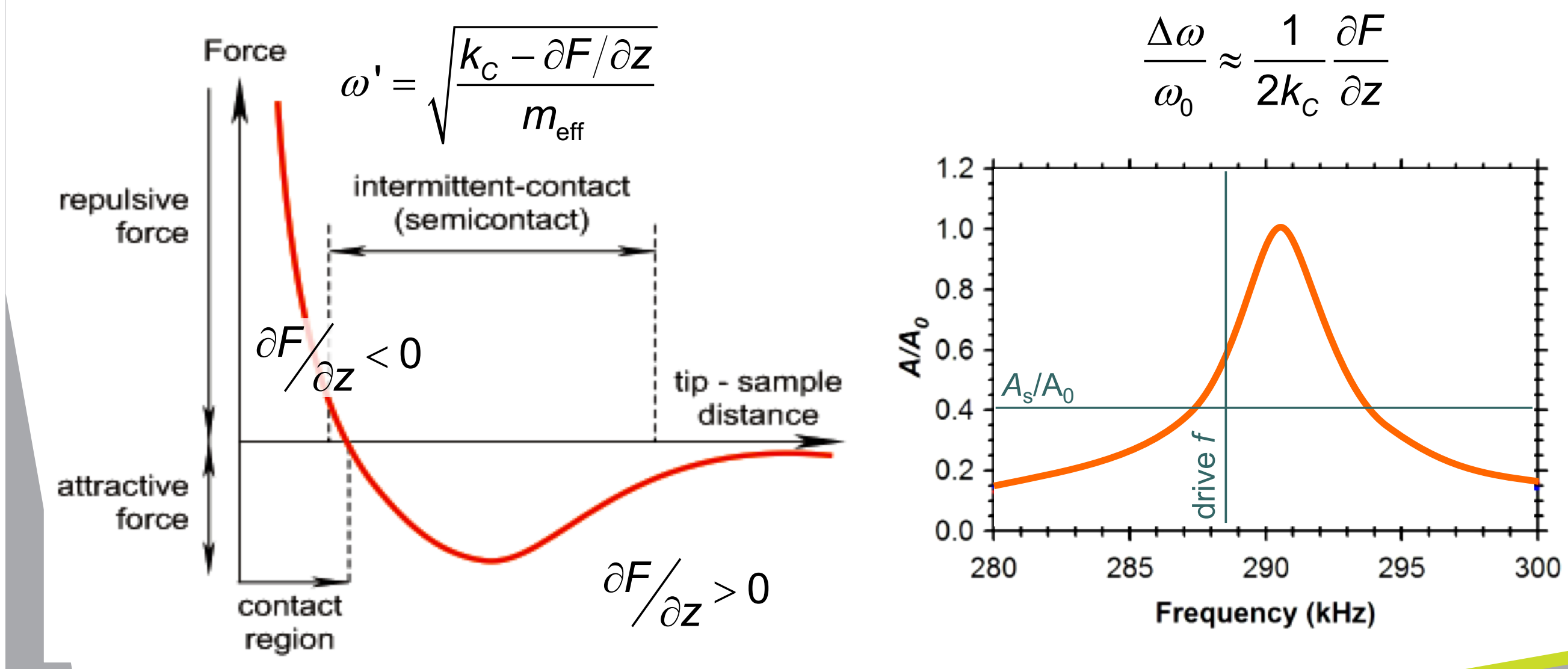


\section{- What are we measuring?}

$\rightarrow$ Linear harmonic oscillator approximation in a force gradient [Magonov et al., Surf. Sci. Lett., 1997; Nysten, 2007]

$$
\Delta \varphi \approx \frac{Q}{k_{C}} \frac{\partial F}{\partial z} \text { if } \omega \sim \omega^{\prime} \sim \omega_{0} \text { and } \frac{\partial F}{\partial z} \ll \frac{k_{C}}{Q}
$$

Pure elastic material with Hertz: $F=\frac{4}{3} E^{*} \sqrt{R} z^{3 / 2} \rightarrow \frac{\partial F}{\partial z} \approx 2 E^{*} a$ $\Delta \varphi \approx \frac{Q}{k_{C}} 2 E^{*} a$

P Phase-lag proportional to the contact stiffness in certain condition $\left(k_{N}<<k_{C} / Q+A_{0} \uparrow\right.$ and $\left.A_{s} \downarrow\right) \rightarrow$ phase images...

but more generally "proportional" to any dissipation (capillarity, viscoelasticity, adhesion, ...) difficult to interpret quantitatively!

$\rightarrow$ High resolution $(<10 \mathrm{~nm})$ and \pm slow imaging (cantilever with high frequency)

$\curvearrowright$ Less tip and sample damages, $\sim$ no friction, ... 


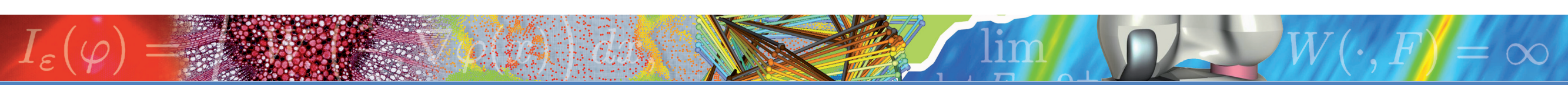

\section{Intermittent contact/tapping ${ }^{\mathrm{TM}} / \ldots$}
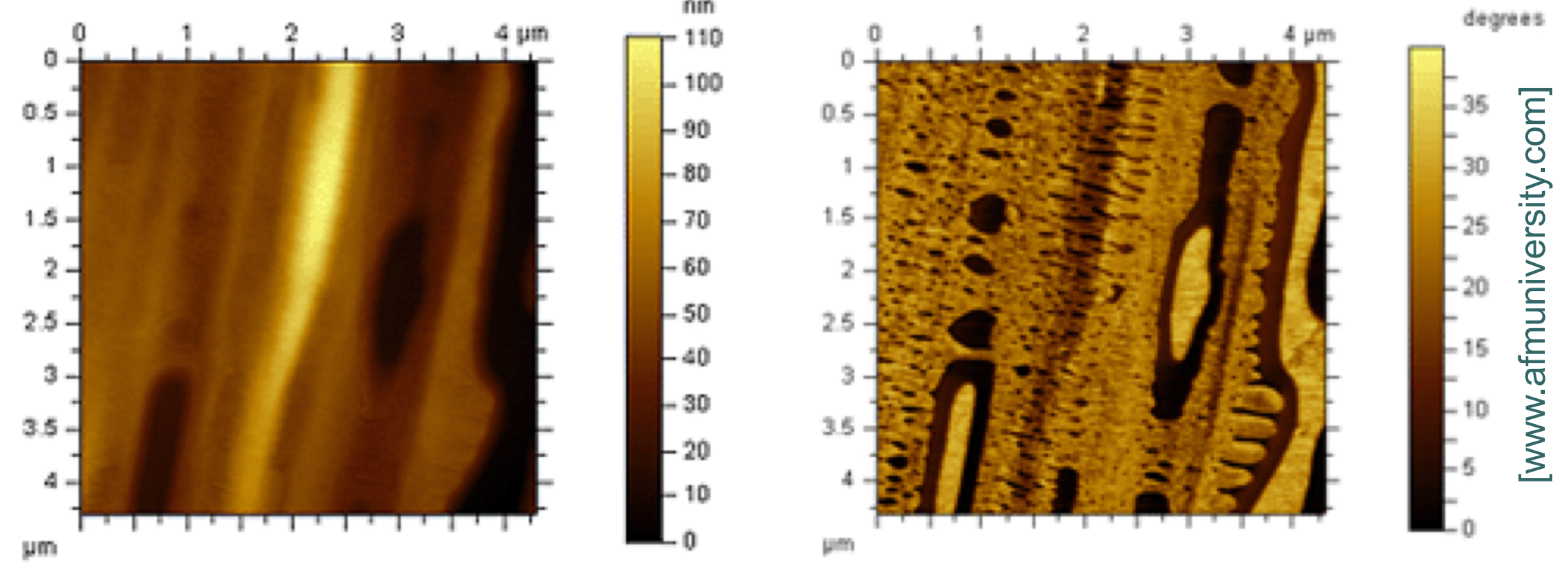

(Figure 6a \& b) Topographic (Left) and phase image (Right) of polydiethysiloxane polymer

Spruce tracheid wood cell wall, AFM probe and condition?
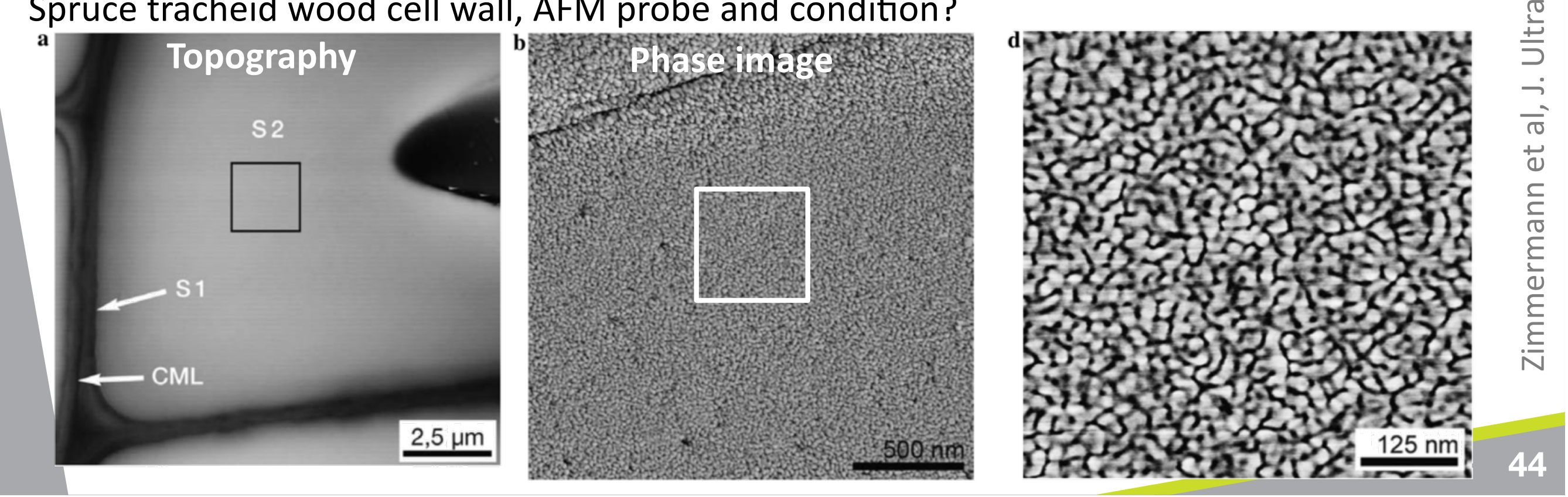


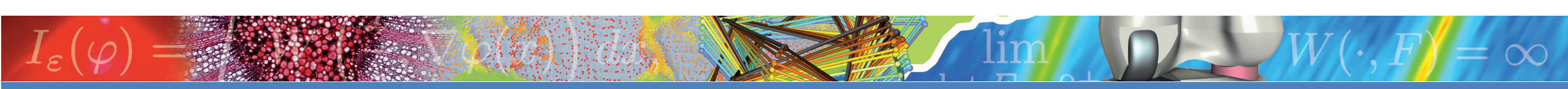

\section{Intermittent contact/tappingTM/...}

Tapping mode (phase image)

$R \approx 4.25 \mathrm{~nm}$

$k_{C} \approx 40 \mathrm{~N} / \mathrm{m}$

$f_{0}=300 \mathrm{kHz}$

Spruce pulp fiber

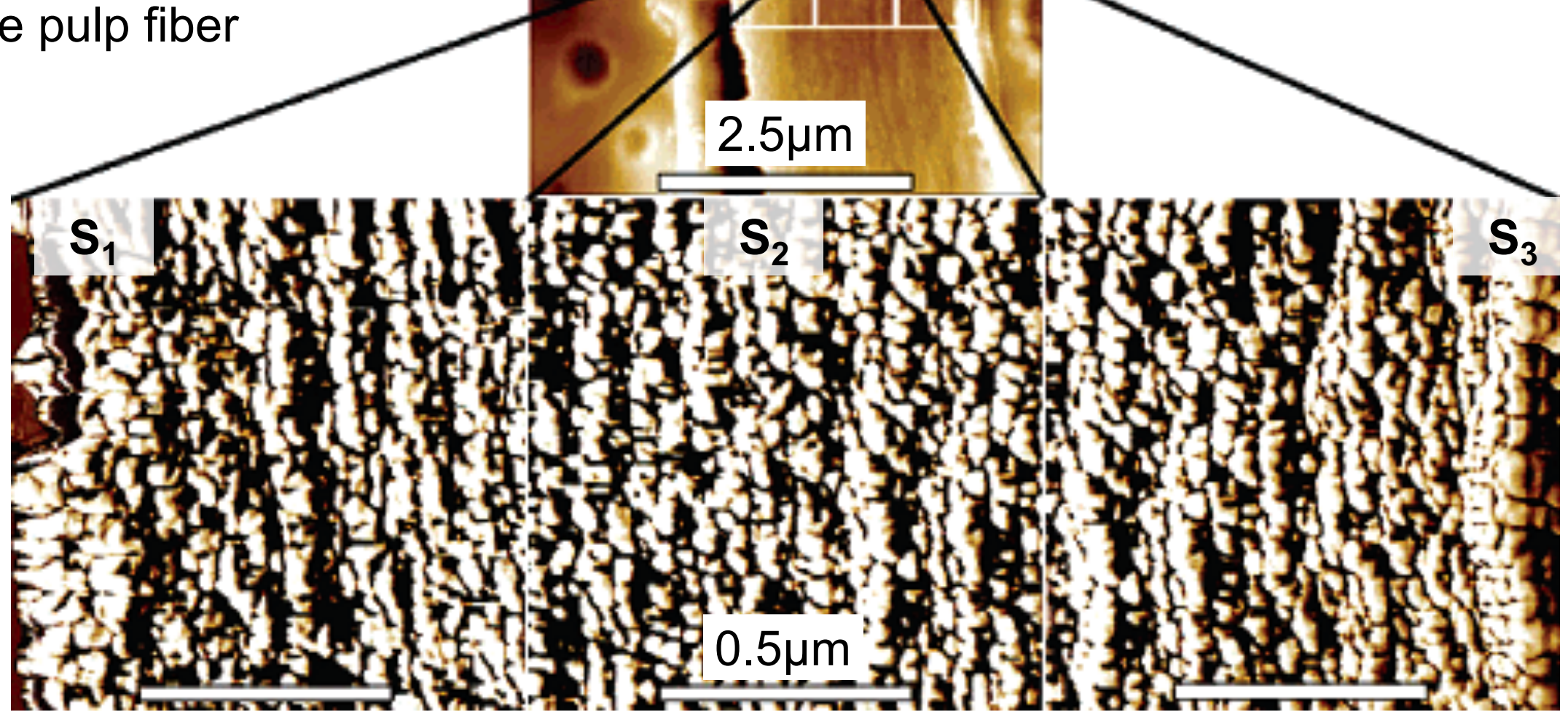

늉

i

$\frac{\infty}{7}$

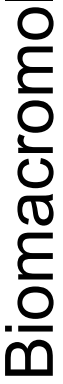

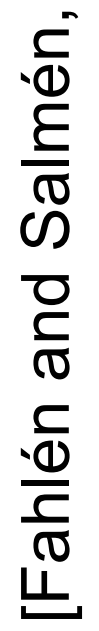




\section{Force-distance curve}

- Van der Waals force, electrostatic force, contact stiffness, adhesion force, ...

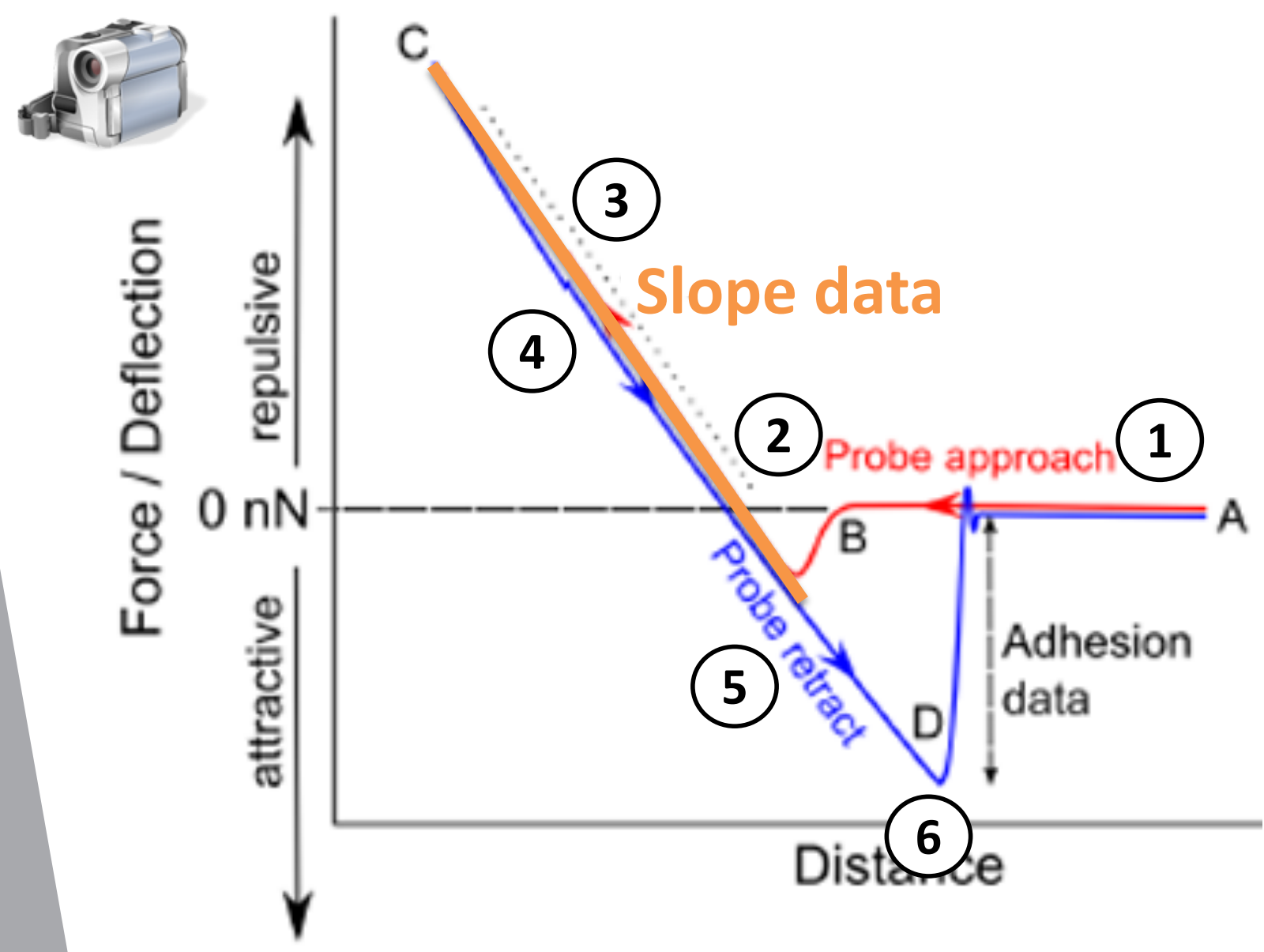

(1)

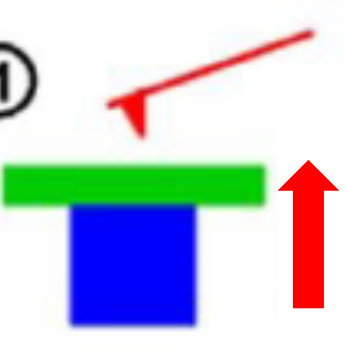

(2)

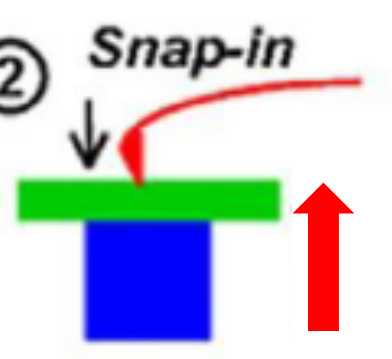

(3) Repulsion

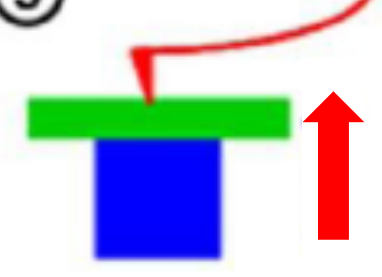

(4) Repulsion

(4)

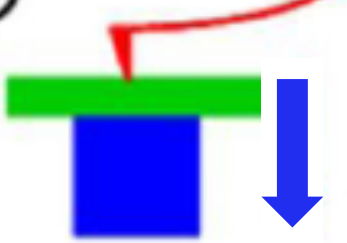

(5) Adhesion

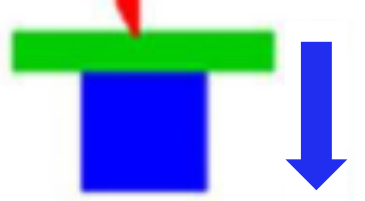

(6)

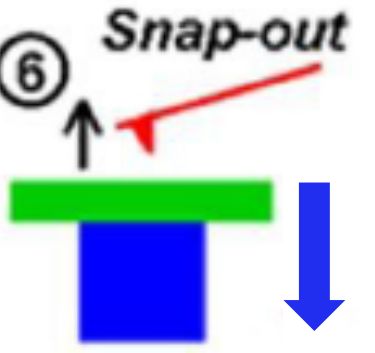

[B. Nysten, 2007] 


\section{Fd - Indentation}

- Decoupling load/displacement

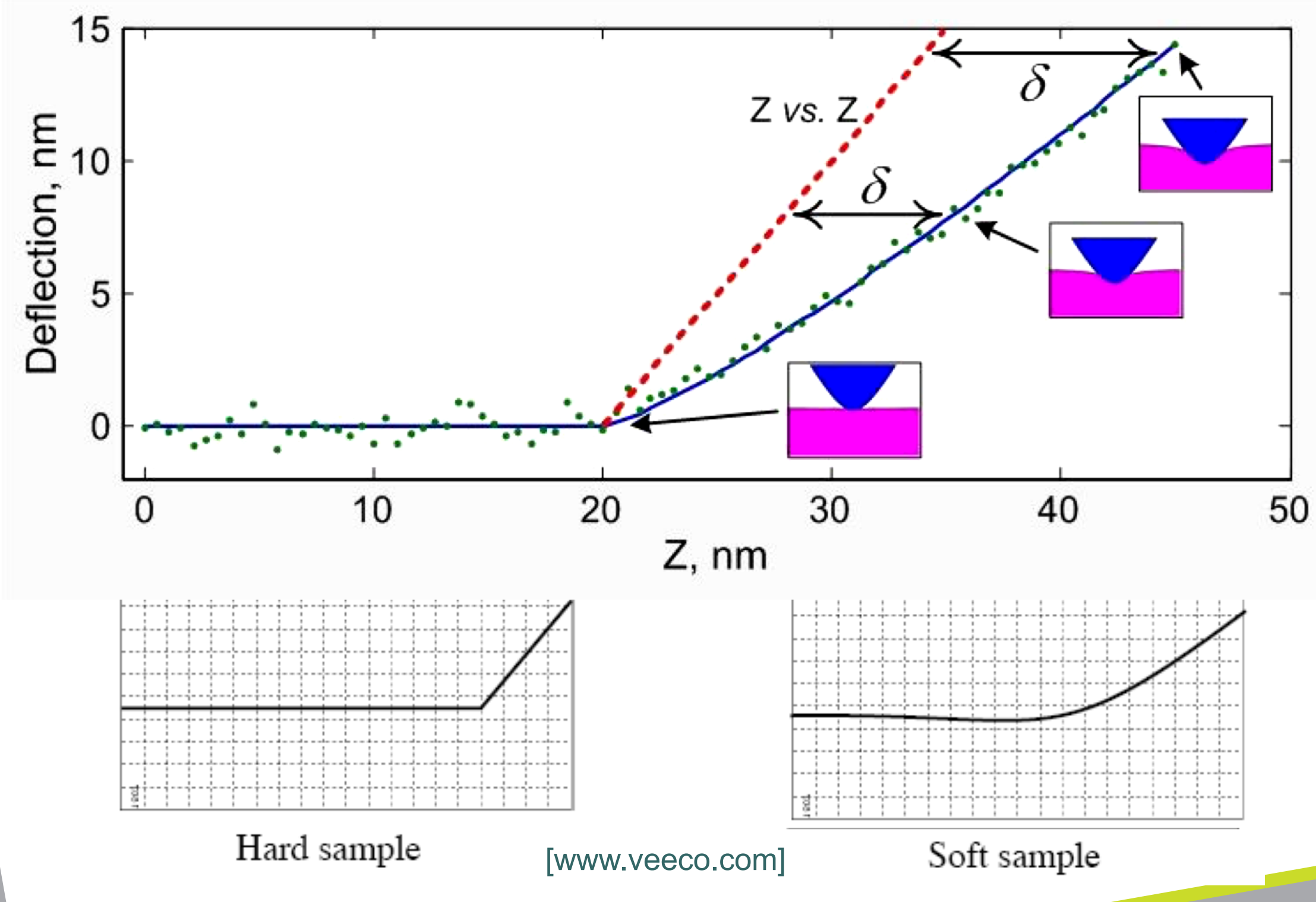




\section{Fd - Indentation}

- Requirements:

$\rightarrow$ Cantilever stiffness

$$
\frac{d}{z}=\frac{1}{1+\frac{k_{C}}{k_{N}}} \mid \begin{aligned}
& k_{N} \gg k_{C} \rightarrow d=z \\
& k_{N} \ll k_{C} \rightarrow d=0
\end{aligned} \quad k_{N} \sim k_{C}
$$

$$
\begin{gathered}
k_{N_{D M T}}=\left(6 M^{2} R\left(F+F_{a d_{D M T}}\right)\right)^{1 / 3} \\
F=k_{c} d=k_{c} \chi V_{A-B}
\end{gathered}
$$

Calibration of the laser/photodetector

C Calibration of the cantilever stiffness

- Measurements of the real tip apex shape + suitable contact model...

Non-normal load (shear) due to cantilever tilt + tip sliding?

- Limited resolution ( $>100 \mathrm{~nm}$ on polymer) due to large tip surface contact area

- Mapping (Force-Volume mode) of the elastic properties is very time consuming $(256 \times 256$ points $=18 \mathrm{~h})$

$\rightarrow$ Force Modulation Mode... PeakForce QNM, QI, etc 


\section{Fd - Indentation}

- Peak Force Quantitative NanoMechanical (QNM) [www.bruker-axs.com]

$\vartheta$ force curve recording at some $\mathrm{kHz}$ with sinusoidal displacement
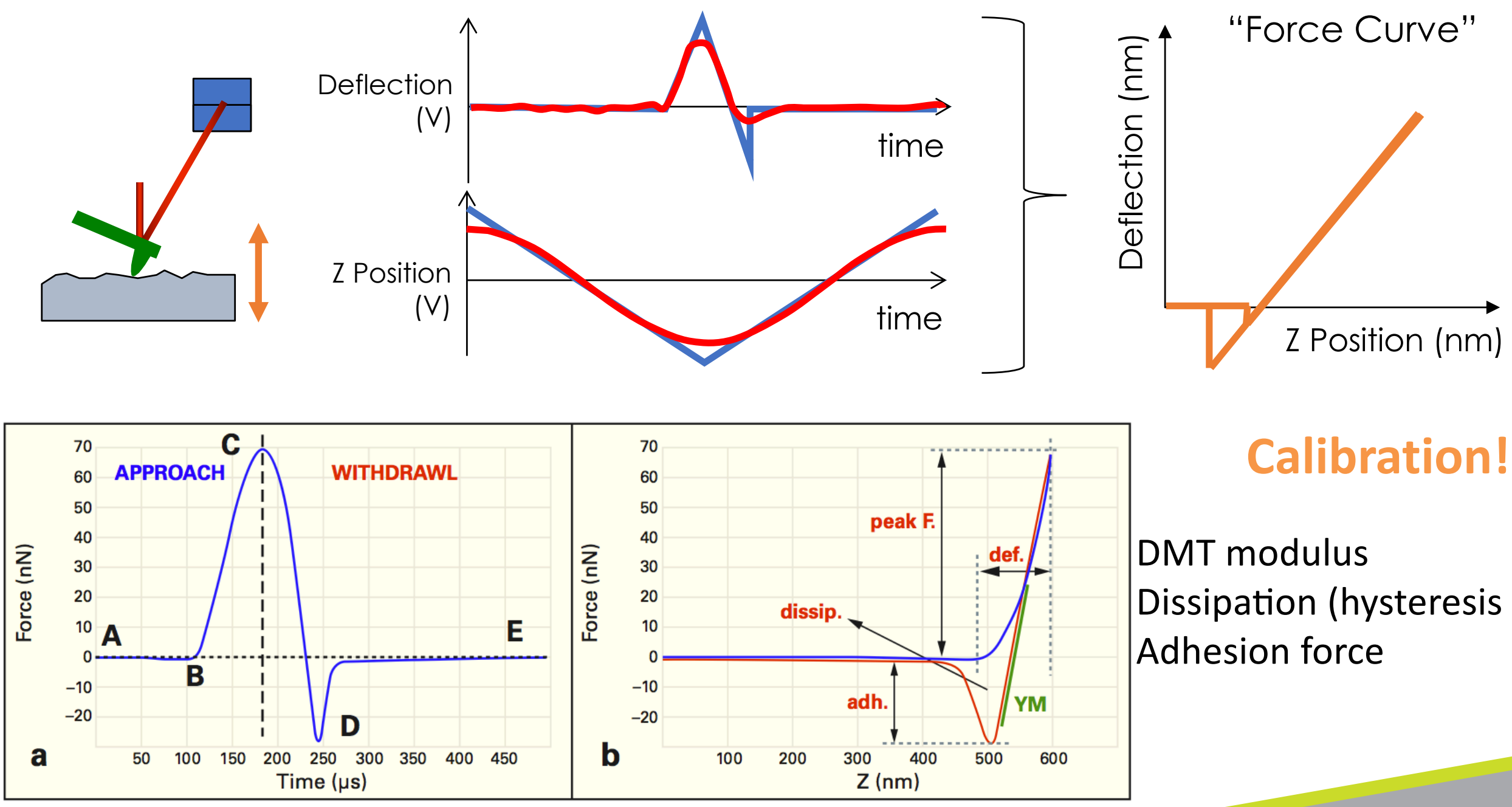

Calibration!

DMT modulus

Dissipation (hysteresis loop) Adhesion force 


\section{Kevlar $^{\circledR}$ Dupont ${ }^{\mathrm{TM}} \mathrm{K} 48$ fibres}

[Arnould et al, Ind. Crops Prod, 2017]

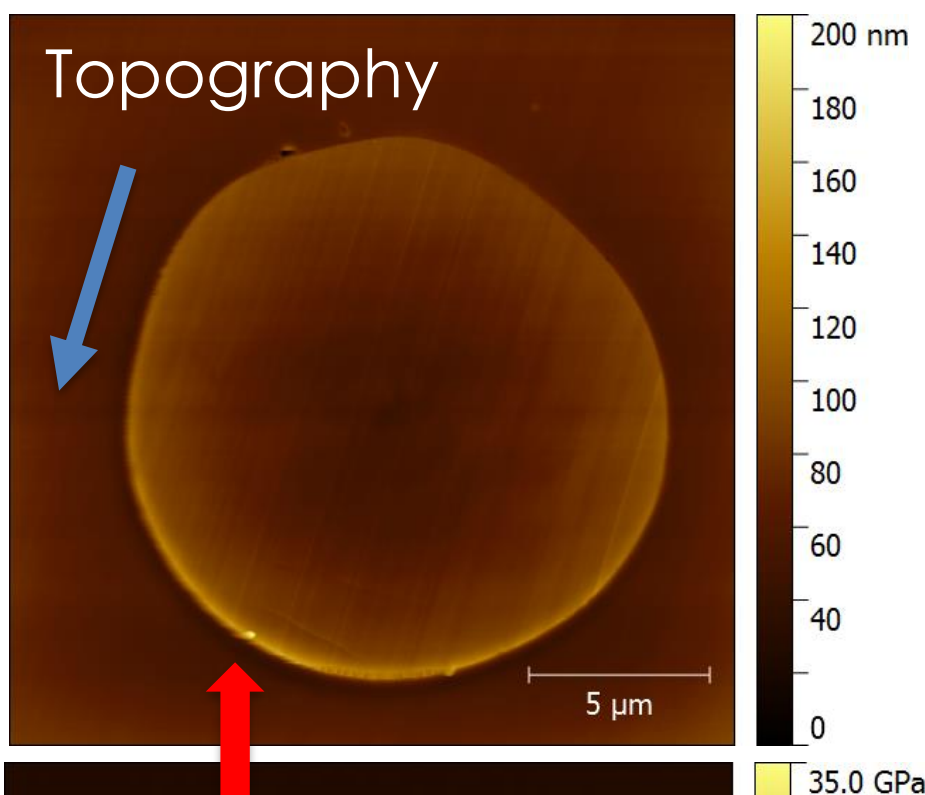

Contc ct modulus

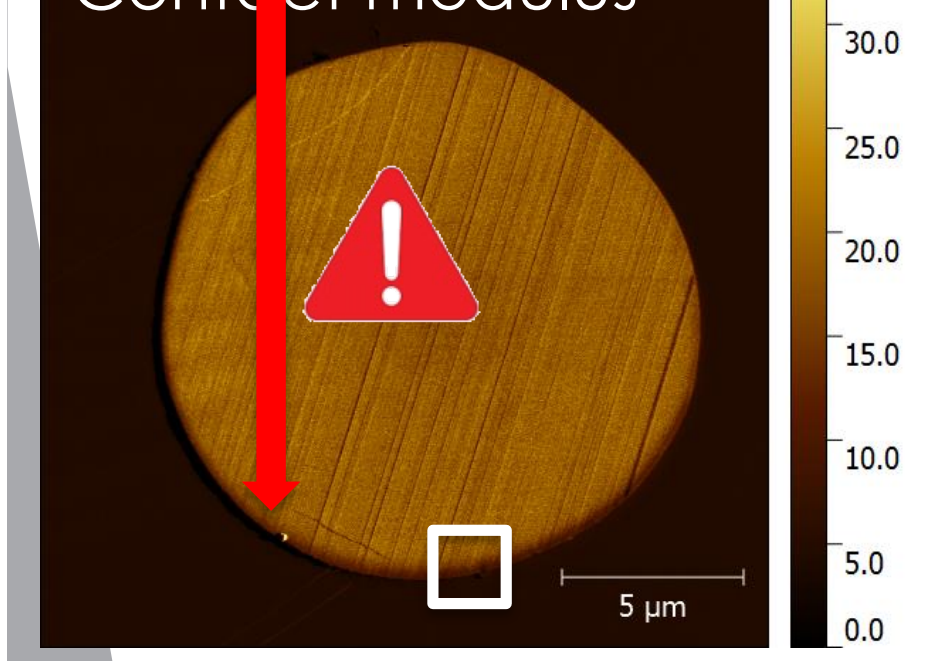

[Wollbrett-Blitz et al, J. Polym. Sci., 2016]

Core:

$\mathrm{E}_{\mathrm{l}}=84 \mathrm{GPa} / \mathrm{E}_{\mathrm{t}}=3 \mathrm{GPa}$

Skin:

$\mathrm{E}_{\mathrm{l}}=85 \mathrm{GPa} / \mathrm{E}_{\mathrm{t}}=0,2 \mathrm{GPa}$

$\mathrm{G}_{\mathrm{lt}}=1,8 \mathrm{GPa} / \mathrm{G}_{\mathrm{tt}}=1,2 \mathrm{GPa}$

$v_{\mathrm{lt}}=0,6 / v_{\mathrm{tt}}=0,25$

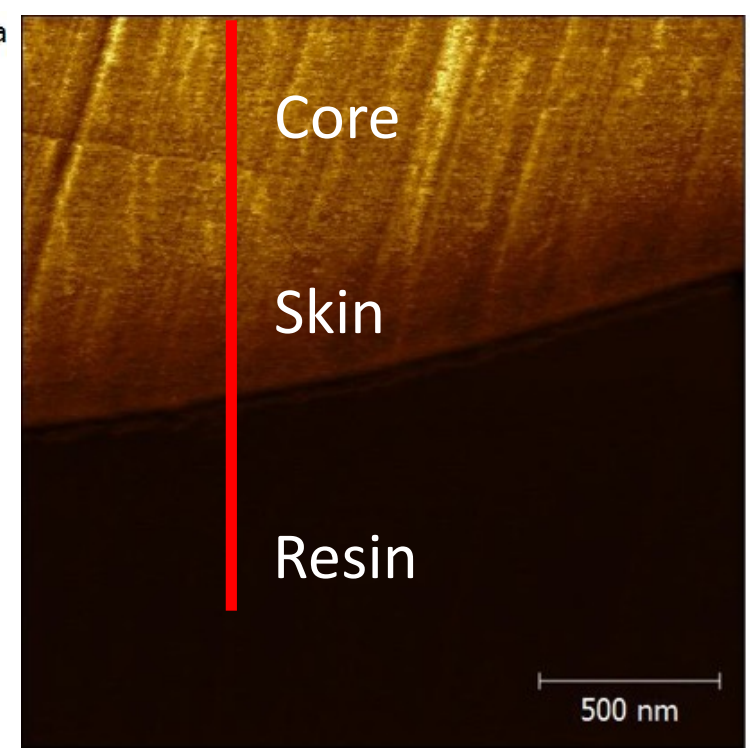

$\mathrm{M}_{\text {core } \mathrm{NI}}=22,8 \pm 1,7 \mathrm{GPa}$

(150nm@ $1 \mu \mathrm{N} / \mathrm{s}+20 \mathrm{~s}+10 \mu \mathrm{N} / \mathrm{s})$

$\mathrm{M}_{\text {core calc }} \approx 21 \mathrm{GPa}$

$\mathrm{M}_{\text {skin calc }} \approx 17 \mathrm{GPa}$

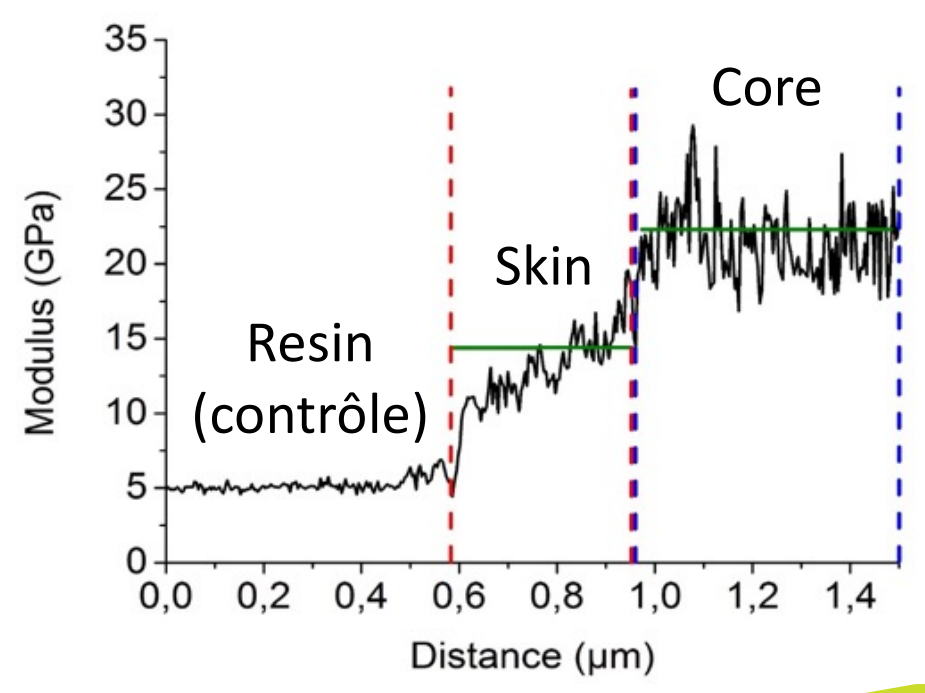

MultiMode 8, PF-QNM, Bruker RTESPA-525, 139 N/m, 32 nm (HOPG), 200 nN 


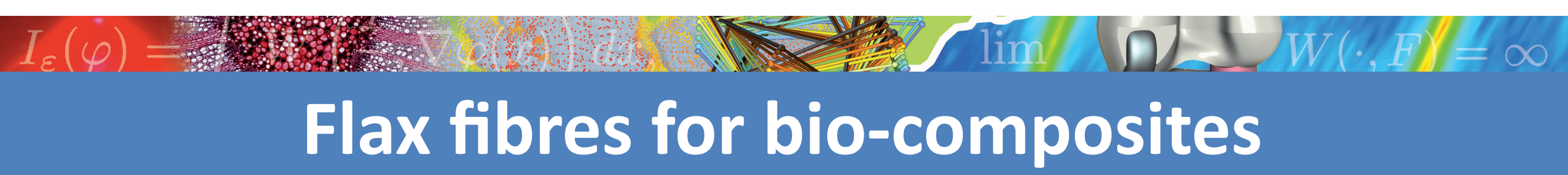

[Arnould et al, Ind. Crops Prod., 2017, 97: 224-228]
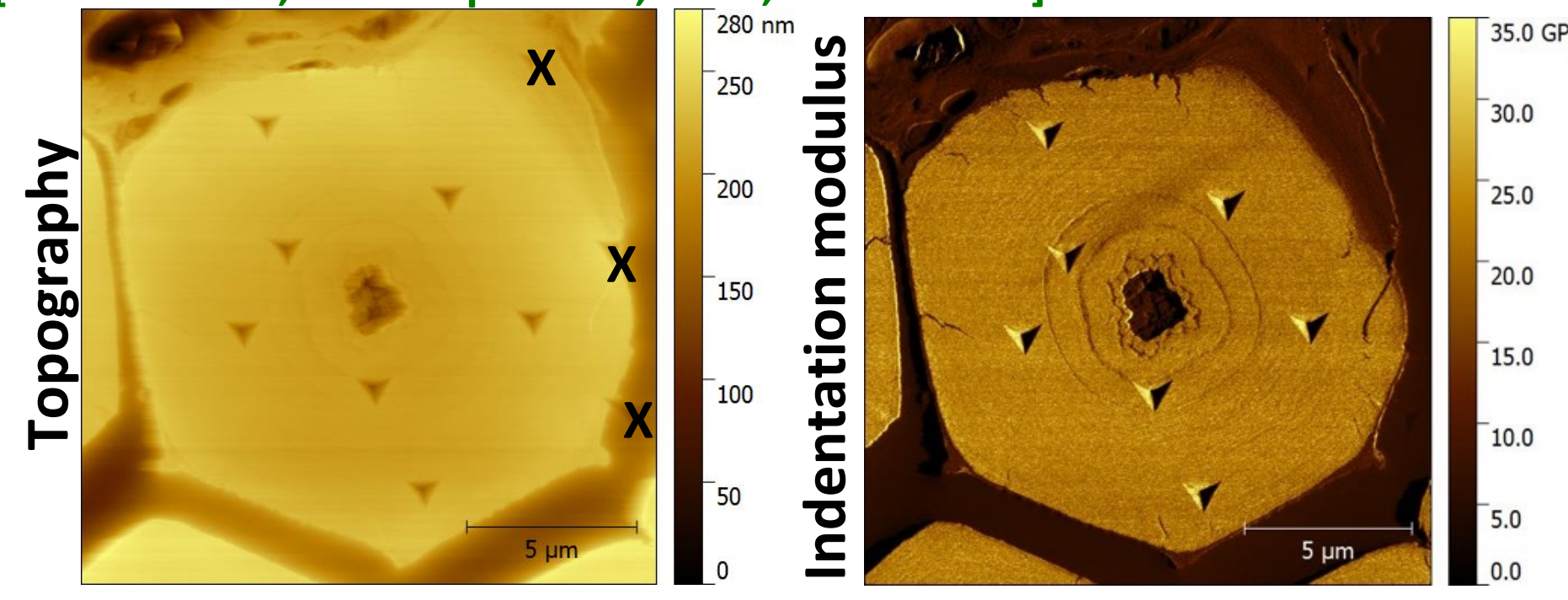

$E_{1}$ fibre $\approx 50-60 \mathrm{GPa}$ !

$\mathrm{M}_{\mathrm{NI} \text { fibre }}=21.3 \pm 2.2 \mathrm{GPa}$ (150 nm @ $1 \mu \mathrm{N} / \mathrm{s}$ $+20 \mathrm{~s}-10 \mu \mathrm{N} / \mathrm{s})$

Effect of processing temperature and fibres confinement in the composite matrix

[Sinsicalco et al, Polym. Test., 2018, 69:91-99]
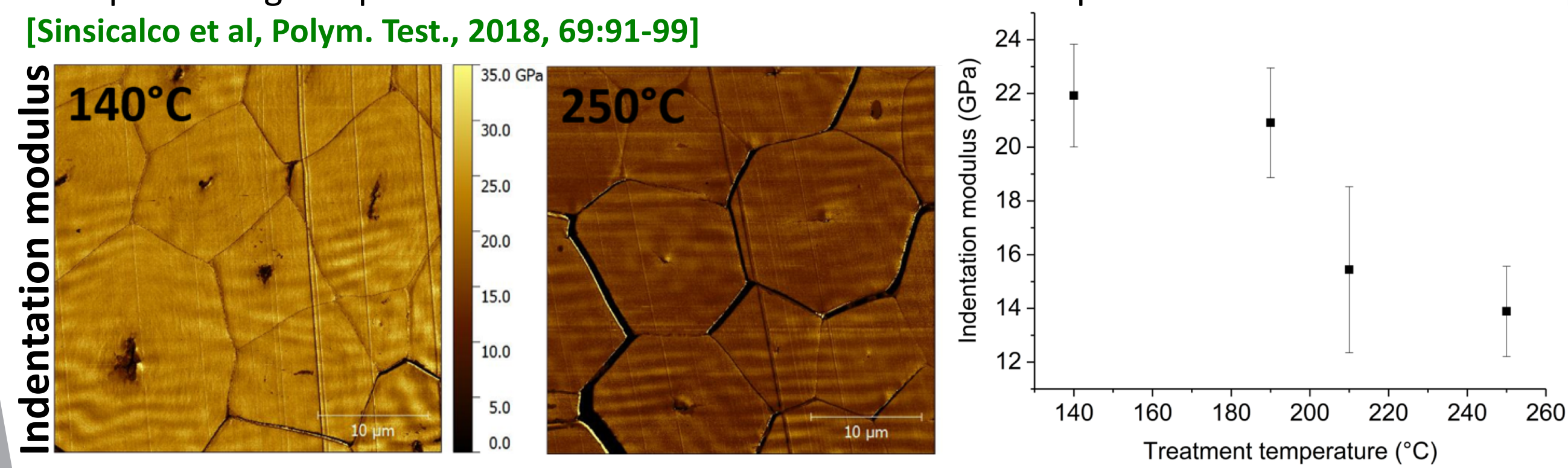

IPDI MultiMode 8, PF-QNM, Bruker RTESPA-525, 139-200 N/m, 9-50 nm (HOPG), 200 nN 51 
$I_{\varepsilon}(\varphi)$ AFM frequency and elastic modulus range...
A

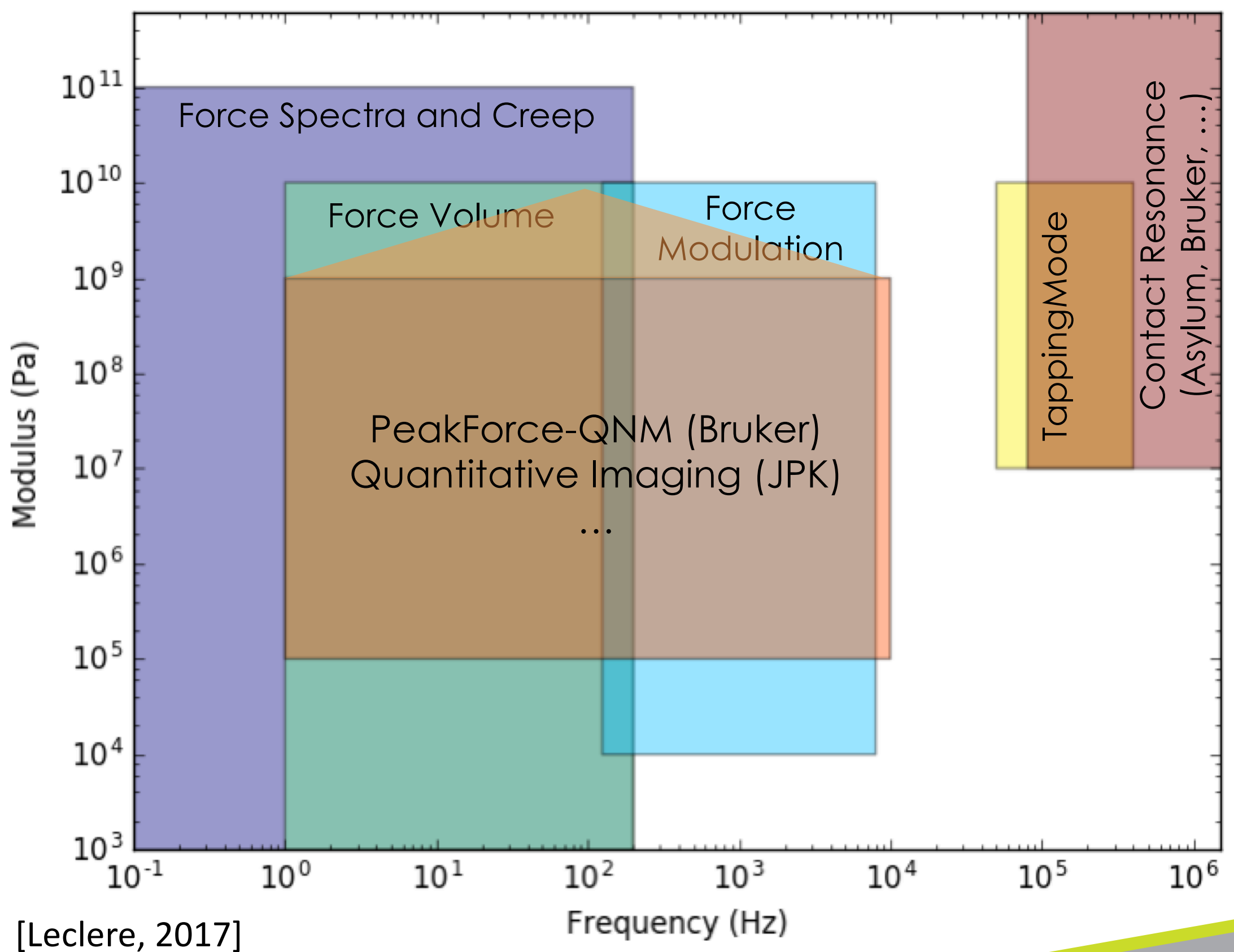




\section{Force-distance curve}

- Van der Waals force, electrostatic force, contact stiffness, adhesion force, ...

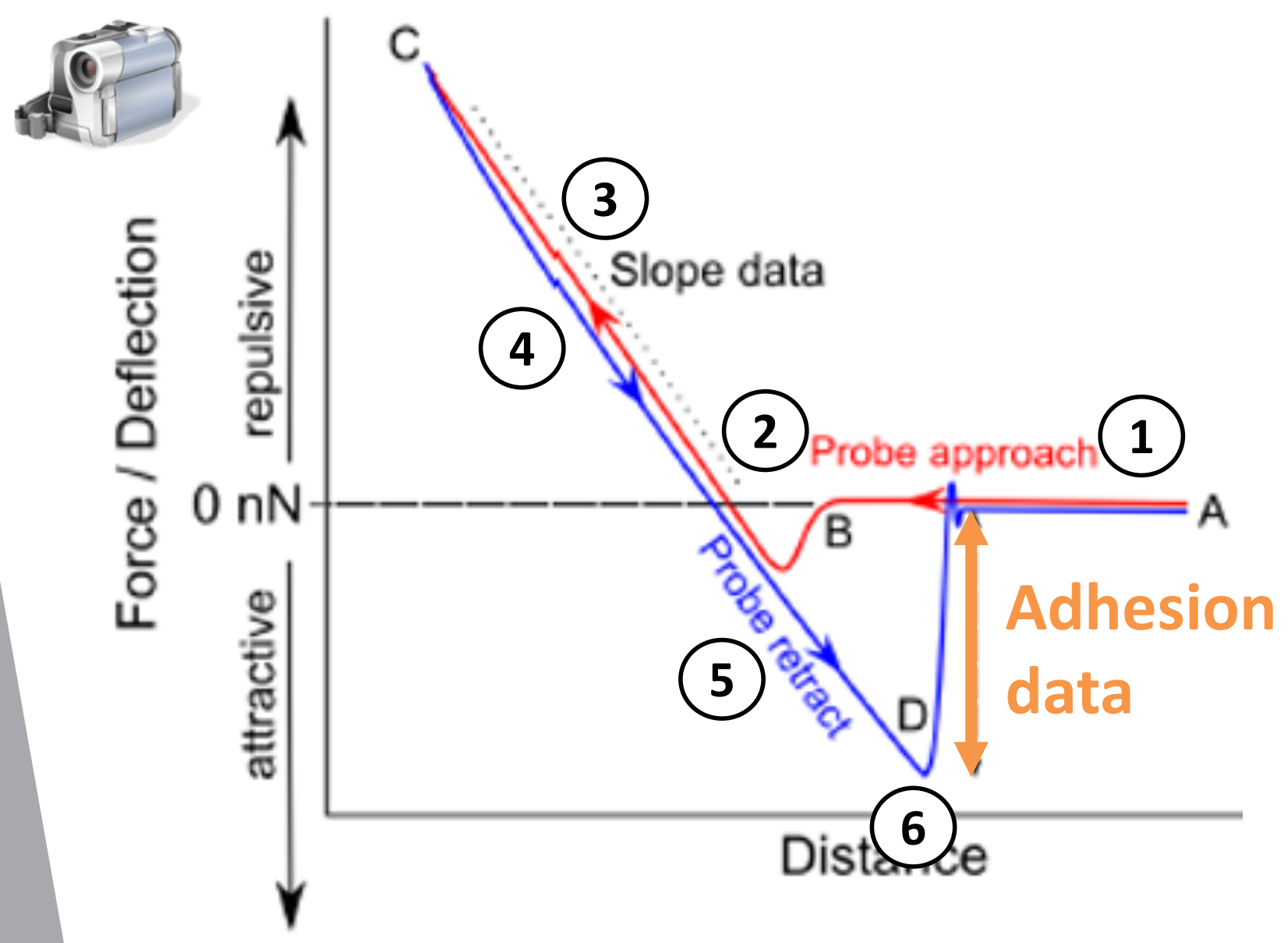

(1)

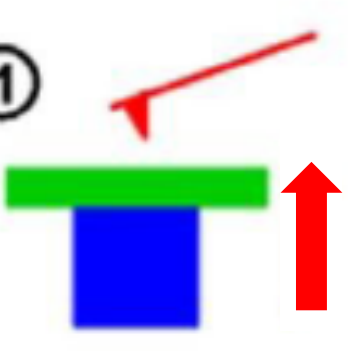

(2)

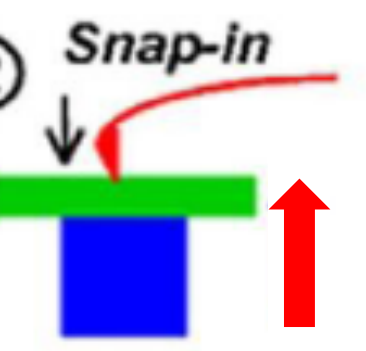

(3) Repulsion

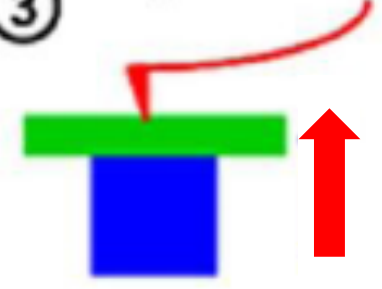

(4) Repulsion

(4)

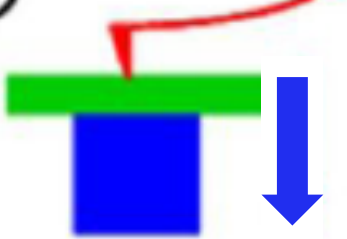

(5) Adhesion

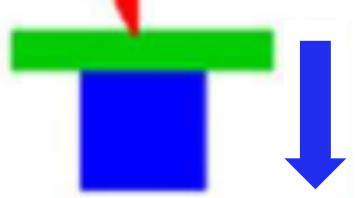

(6)

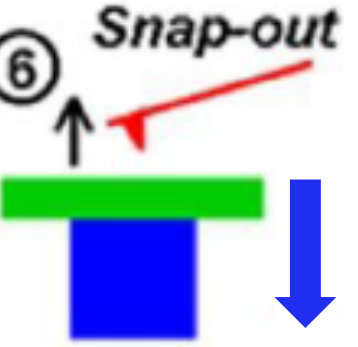

[B. Nysten, 2007] 
- Influence of surface roughness and tip geometry

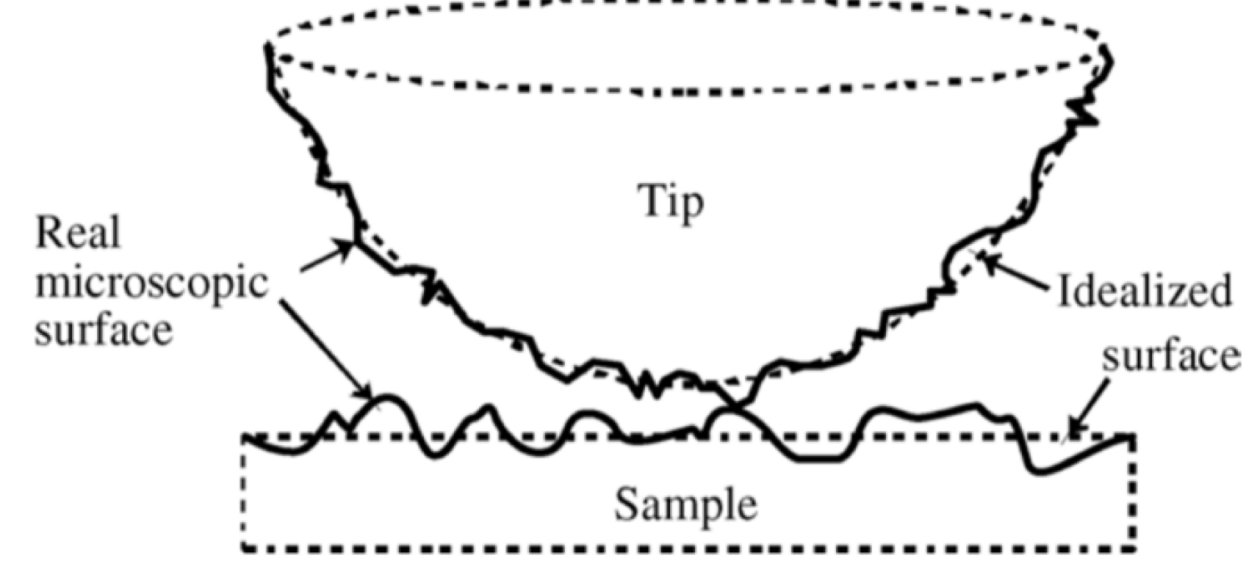

Butt et al, Surf. Sci. Rep. 2005, 59, 1-152

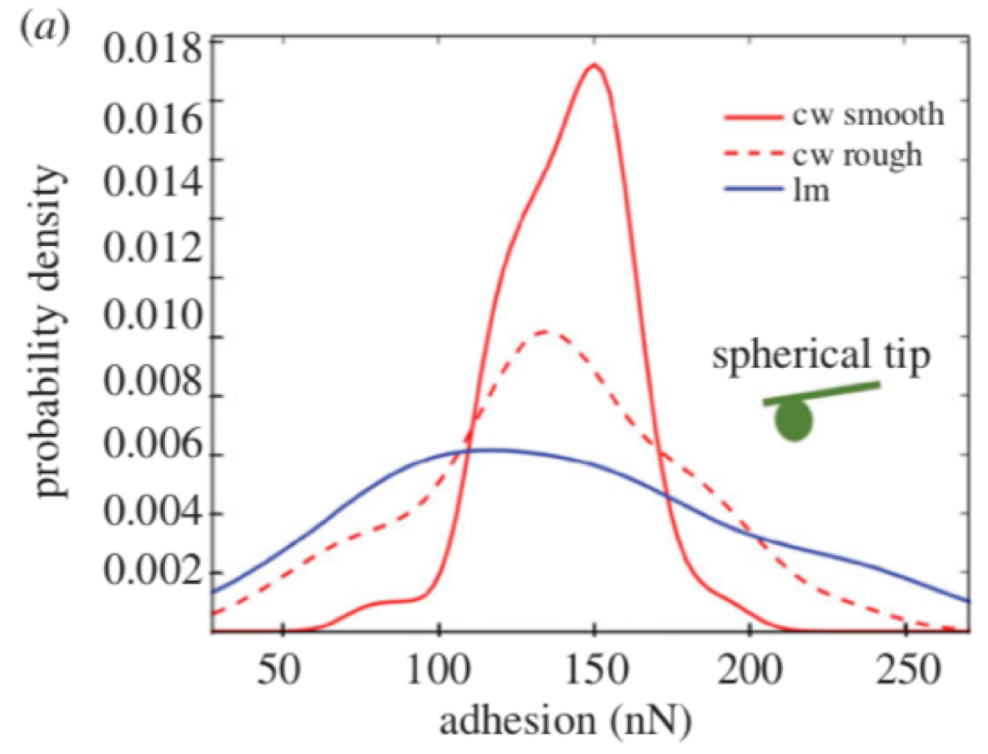

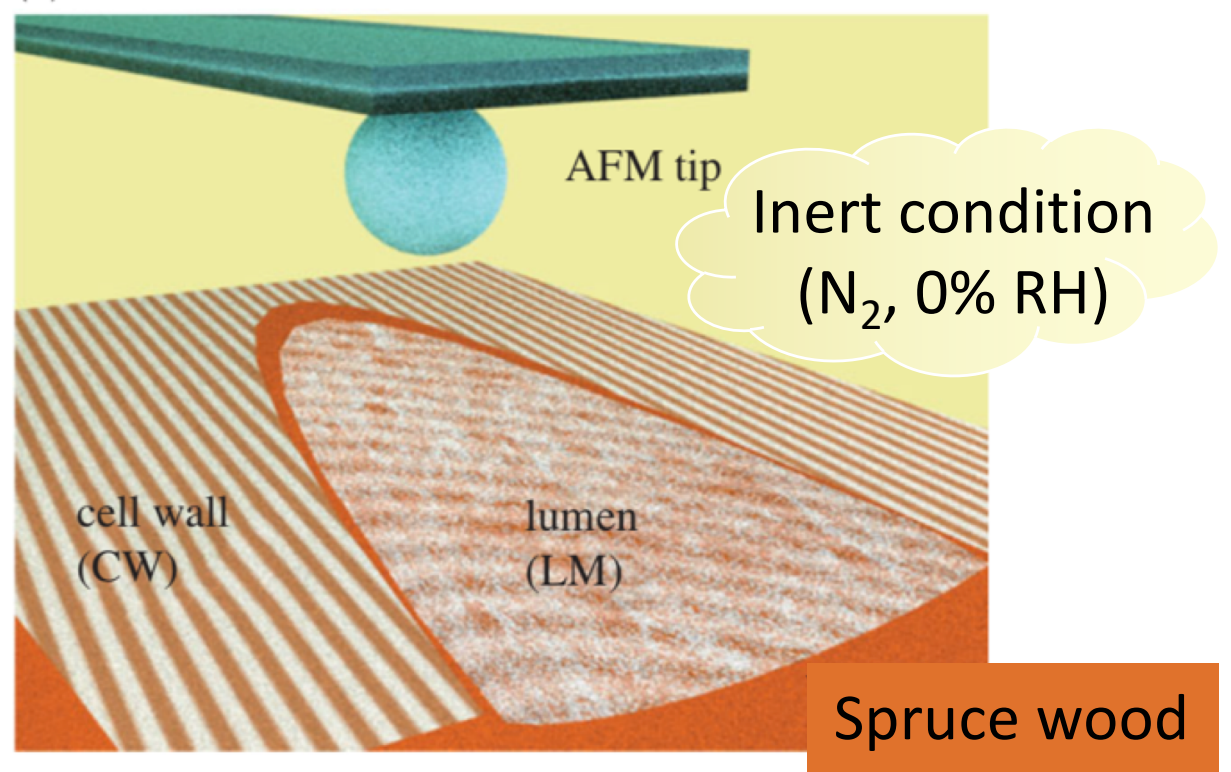

(b)

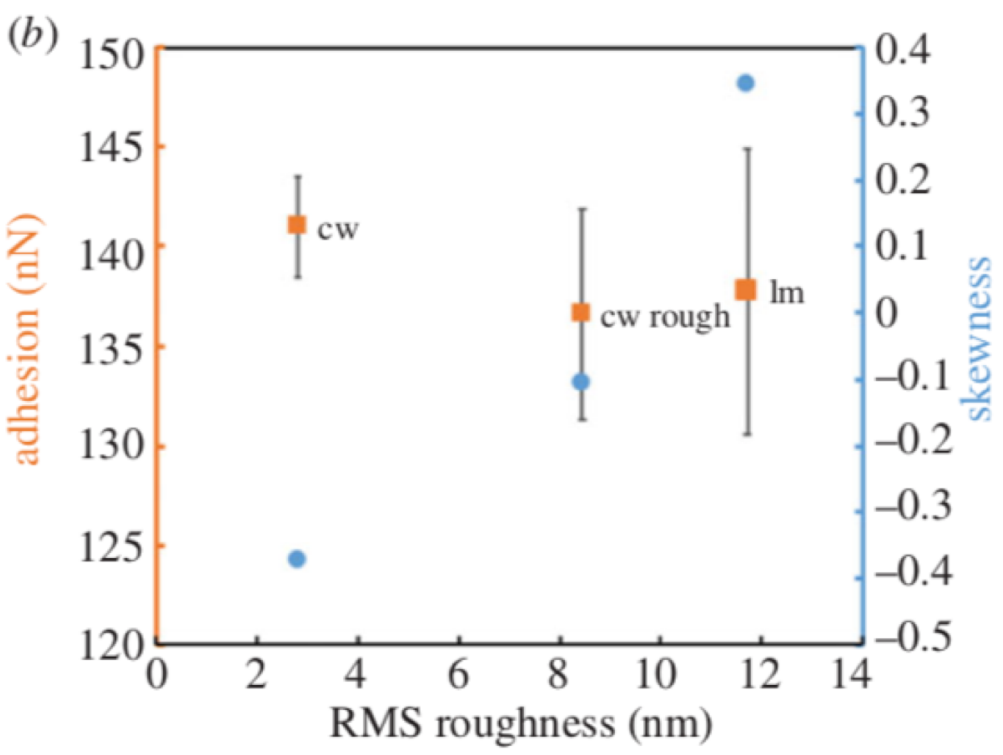




\section{Adhesion force spectroscopy}

\section{- Variability in surface polarity and aging}

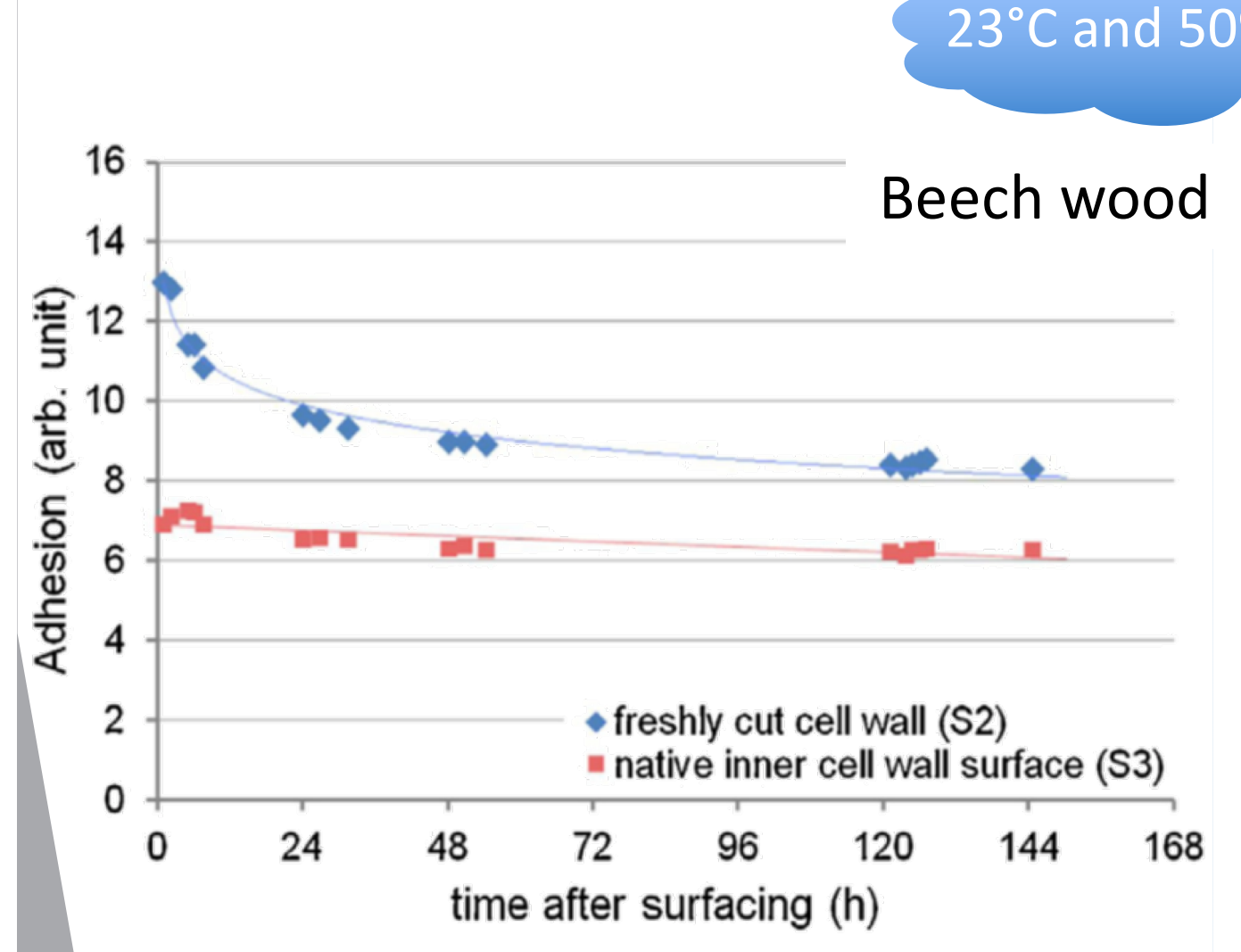

Frybort et al, Colloids and Surfaces A, 457 (2014) 82-87 adhesion force

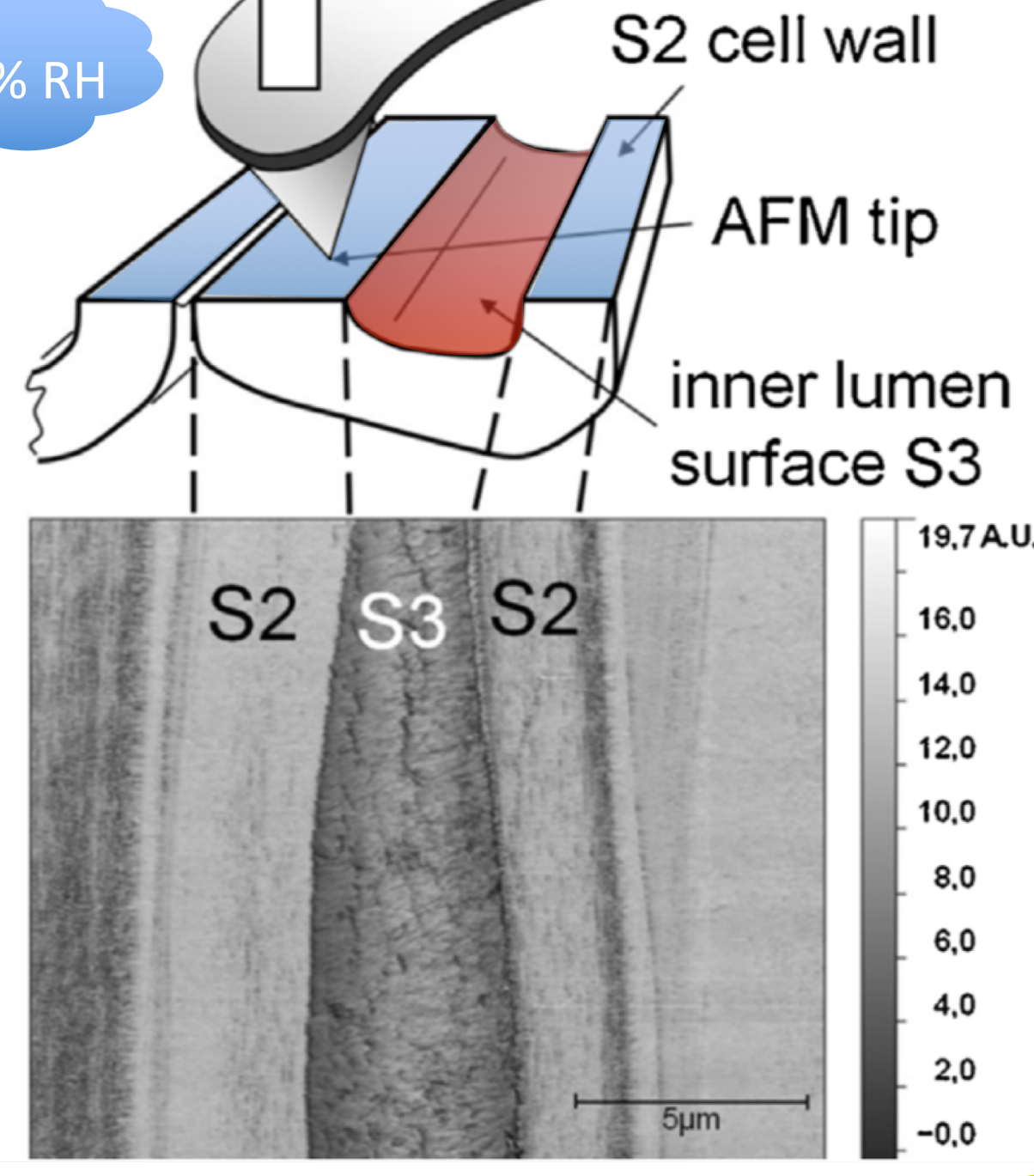




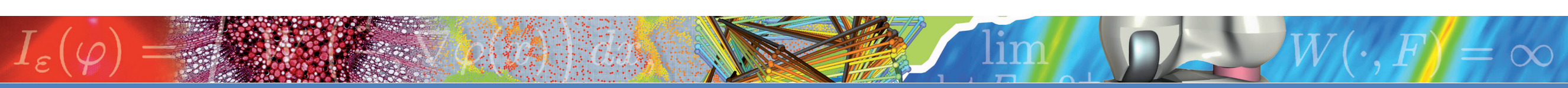
Adhesion force spectroscopy

- Effect of surface treatment
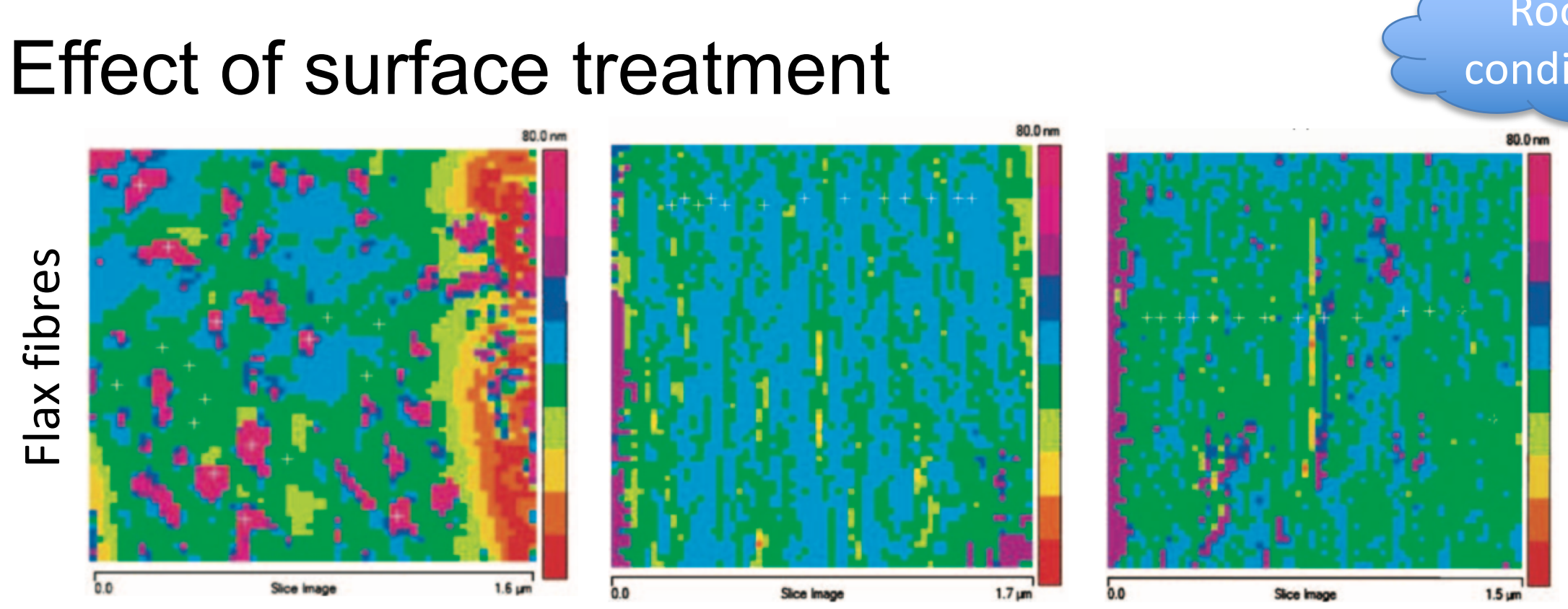

Room
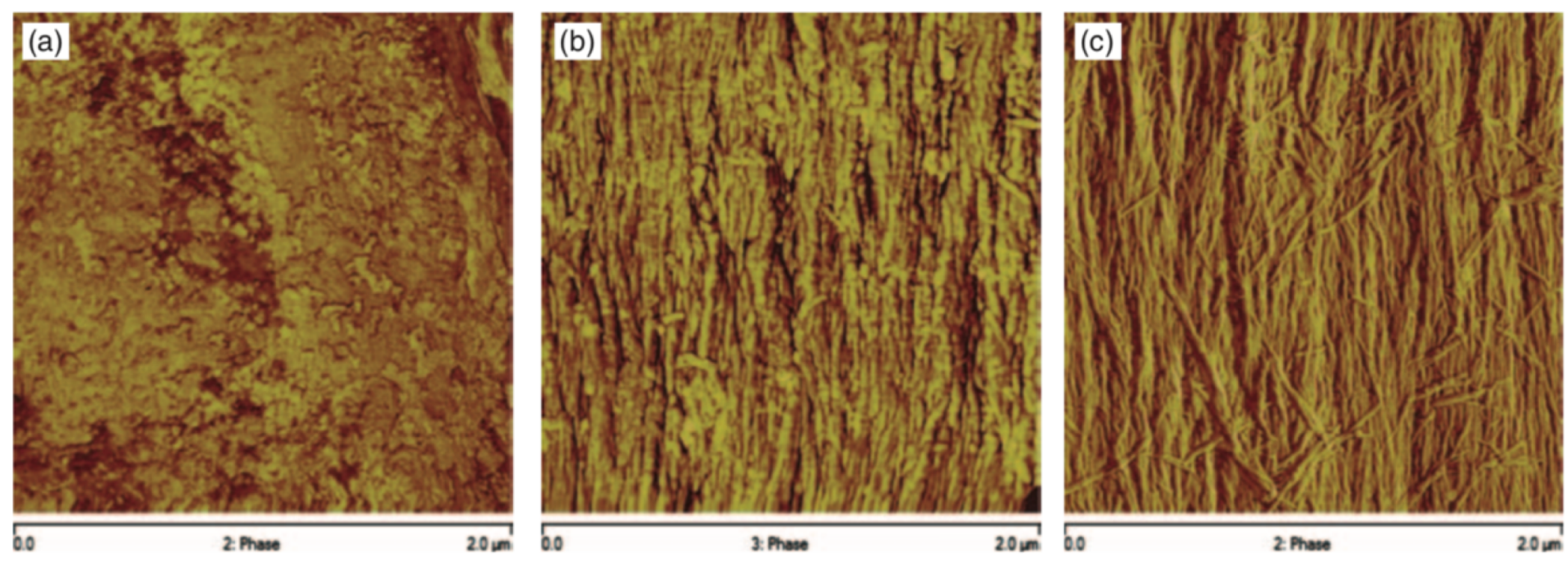

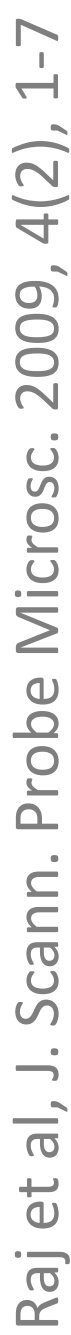

TM-AFM phase image of (a) raw flax fibre, (b) $\mathrm{NaOH}(10 \%)$ treated flax fibre, (c) Enzyme treated (18 h) flax fibre. 
- Tip functionalisation - Chemical force microscopy
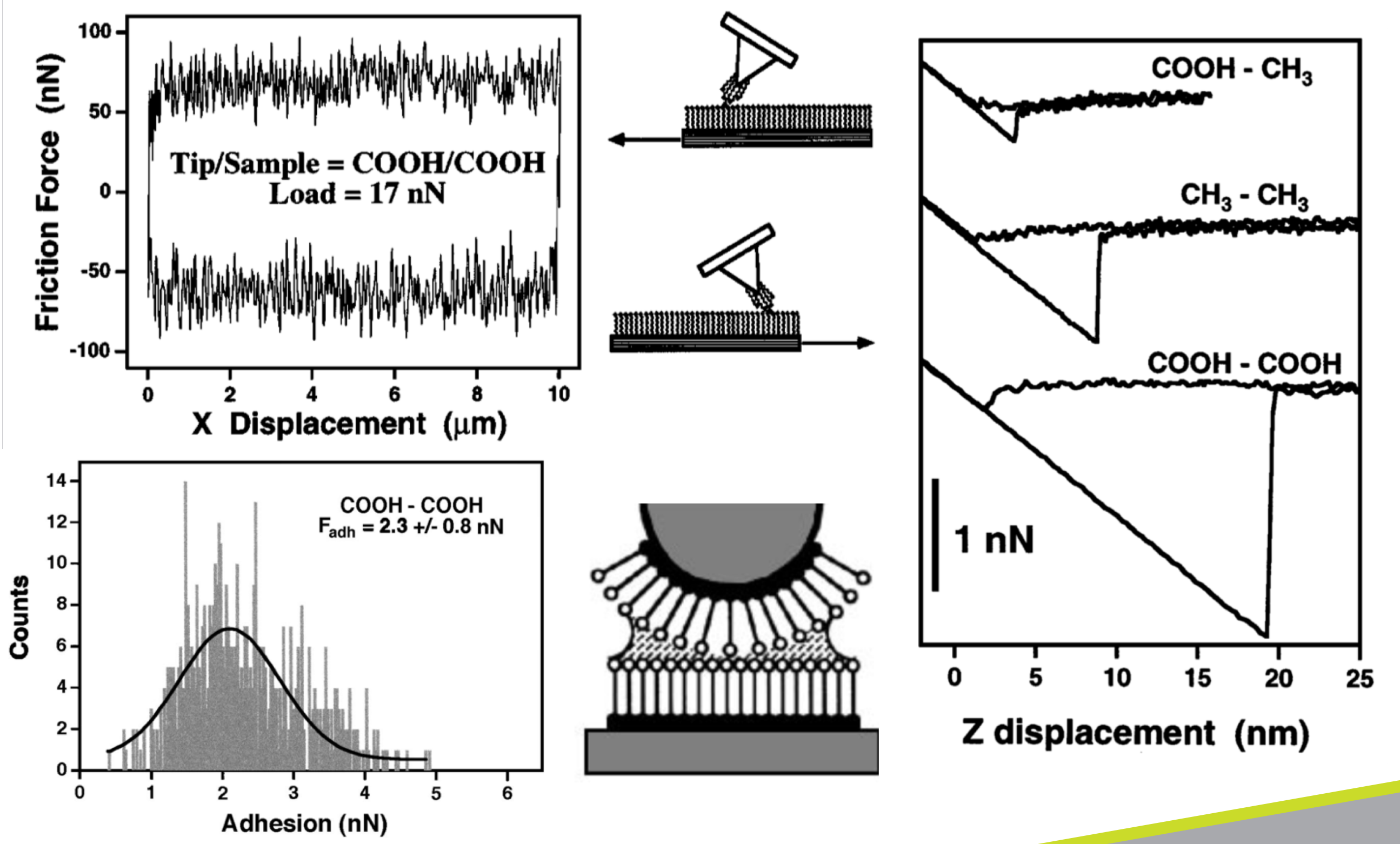


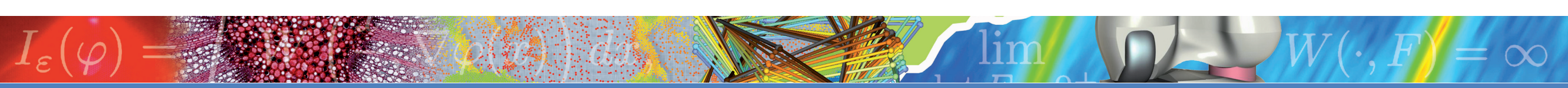

\section{Adhesion force spectroscopy}

- Tip functionalisation - Molecular force microscopy
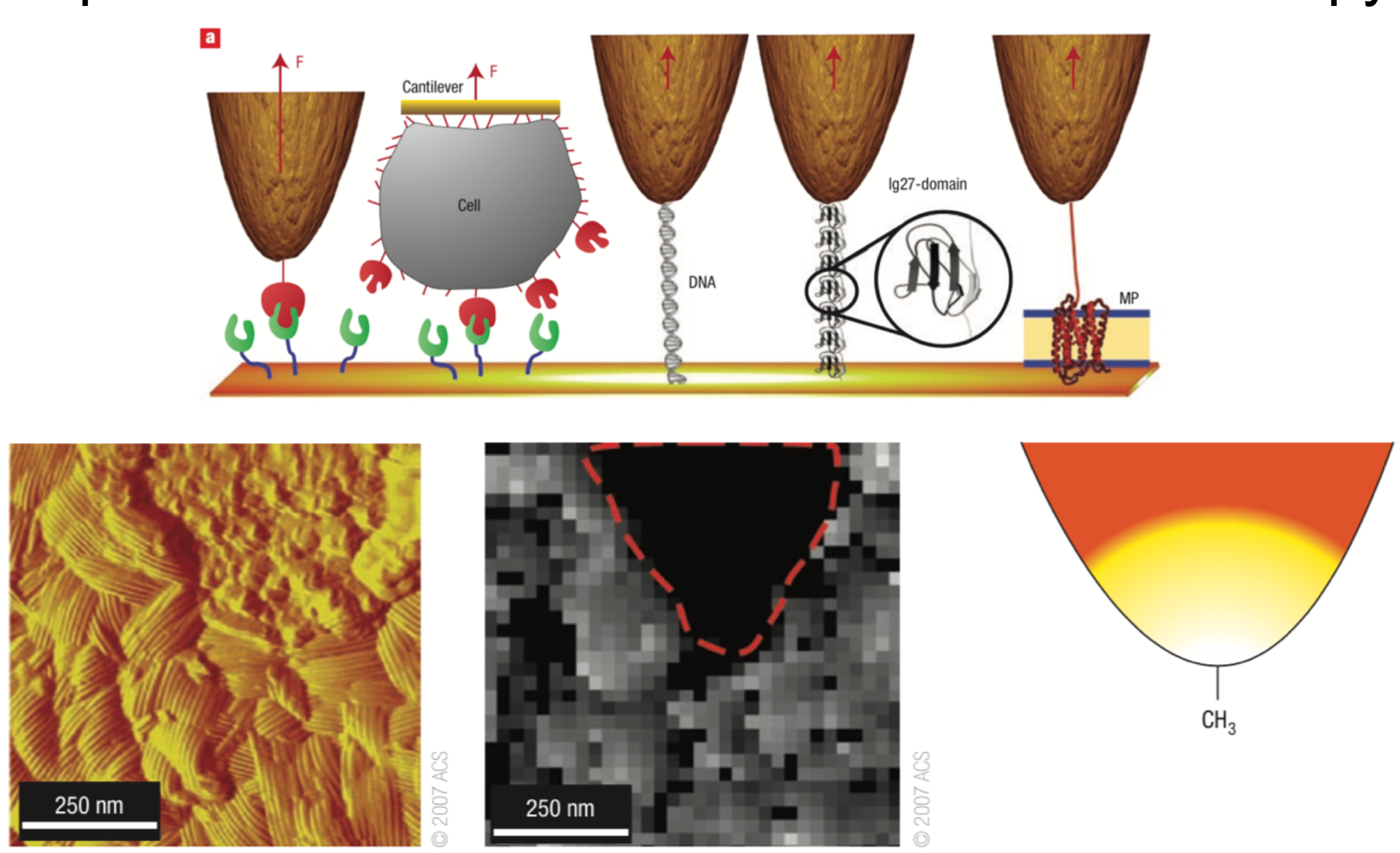

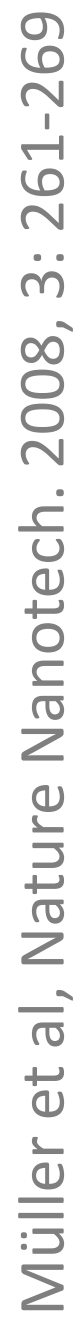

Fungus Aspergillus fumigatus 


\section{Outline}

- Brief history and basic principles of scanning probe microscopy

- AFM principle and typical set-up

- Tip probe-sample surface interactions

- Tip and cantilever

- Deflection sensor and scanner

- Whole set-up

- Contact mechanics

- Operating modes

- Contact

- Intermittent

- Force-distance curve and mechanical characterisation modes Image analysis and artefacts

- Sample preparation 


\section{AFM artifacts}

\section{- Images dilation due to the "finite" tip shape}
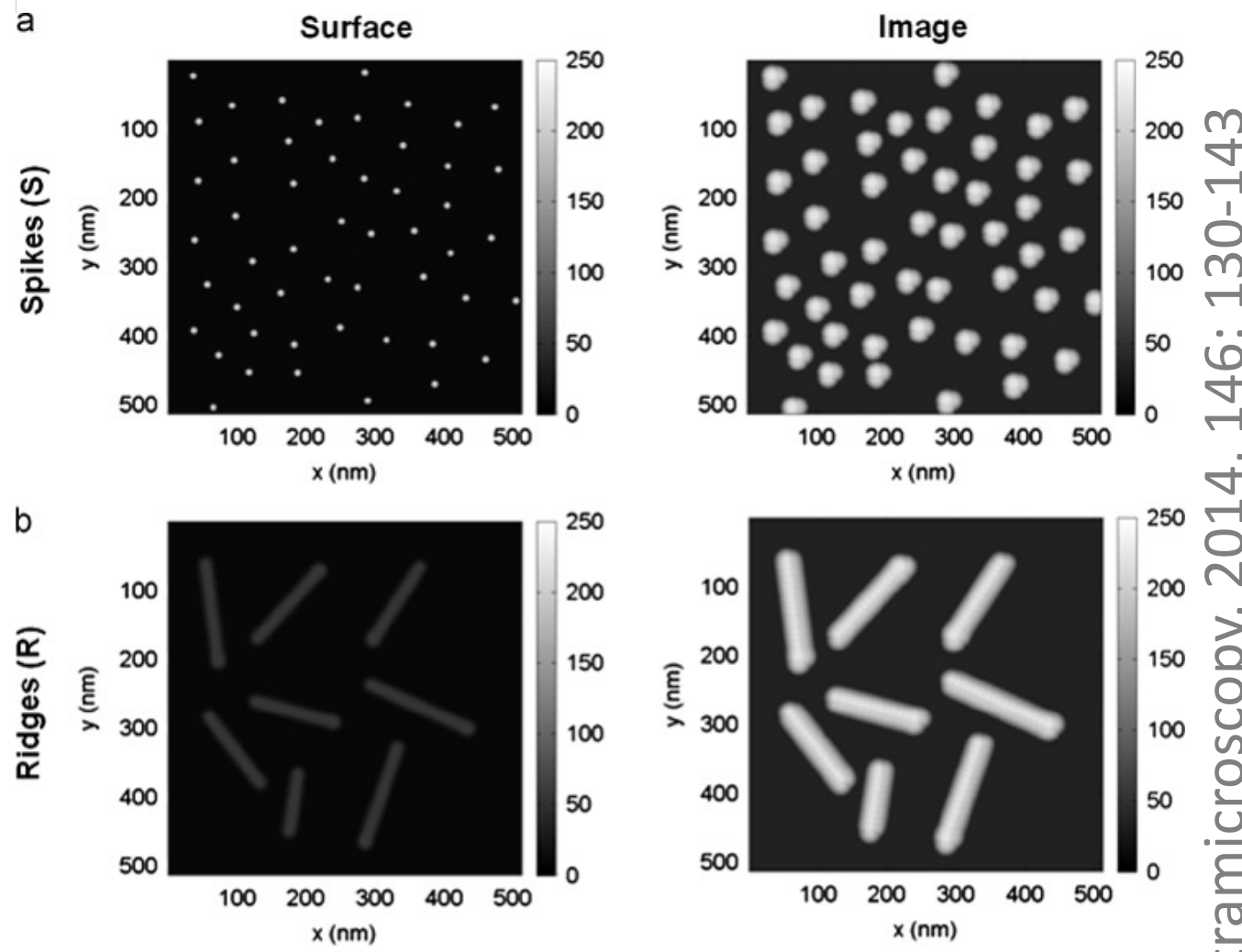

C

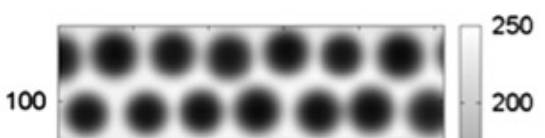

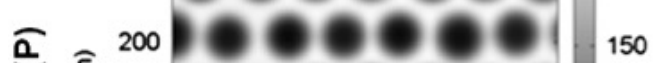

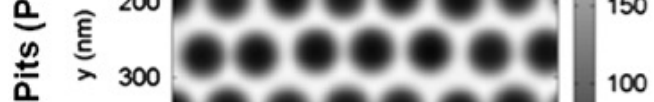

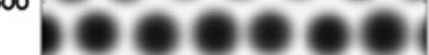
$400 \mathrm{C}-\mathrm{e}-\mathrm{C}=$ $500 \mathrm{e}$ -
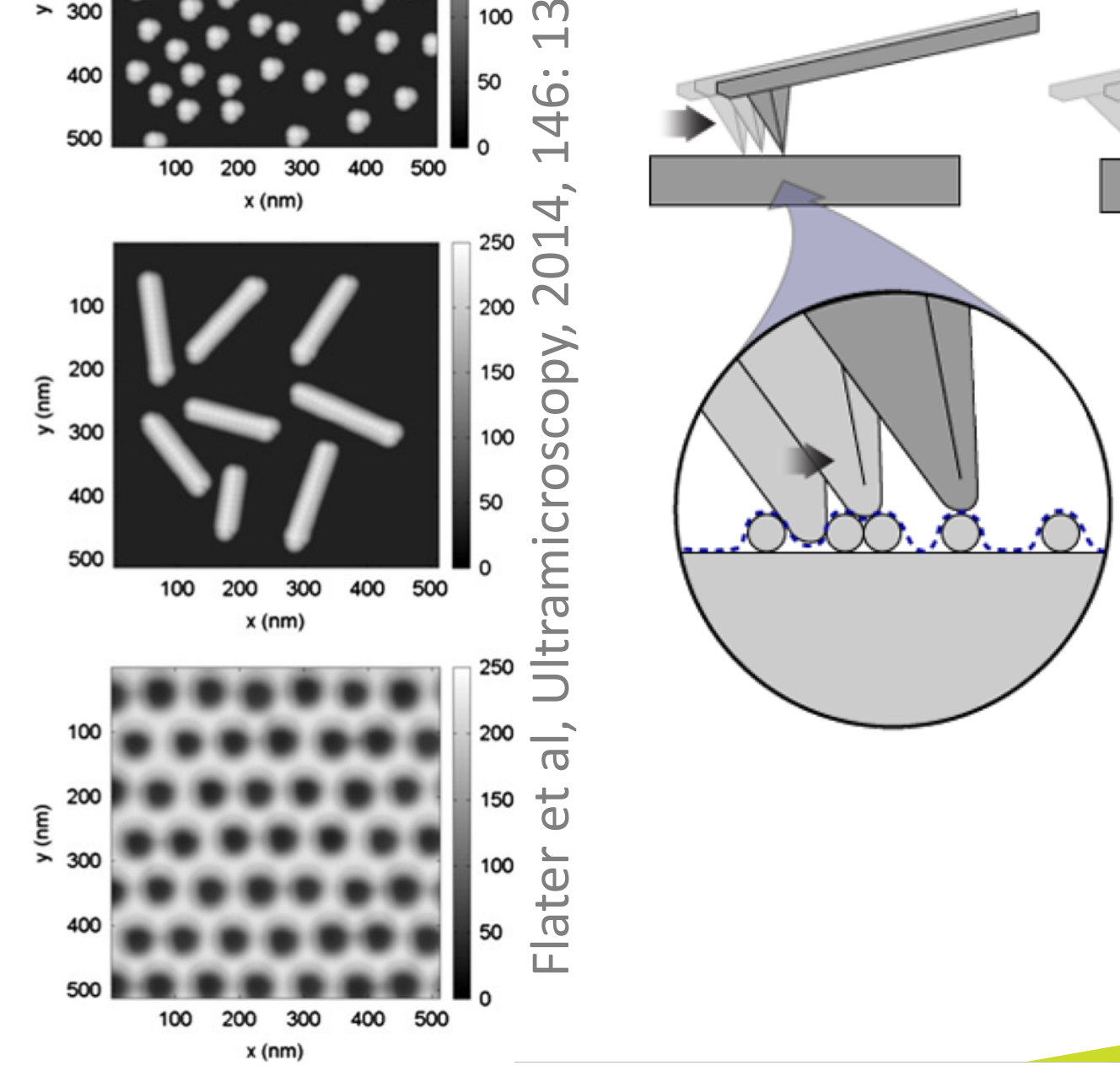


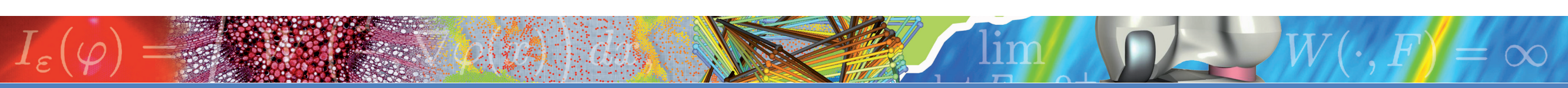

\section{AFM artifacts}

- Images dilation due to tip shape default, dirt or wear
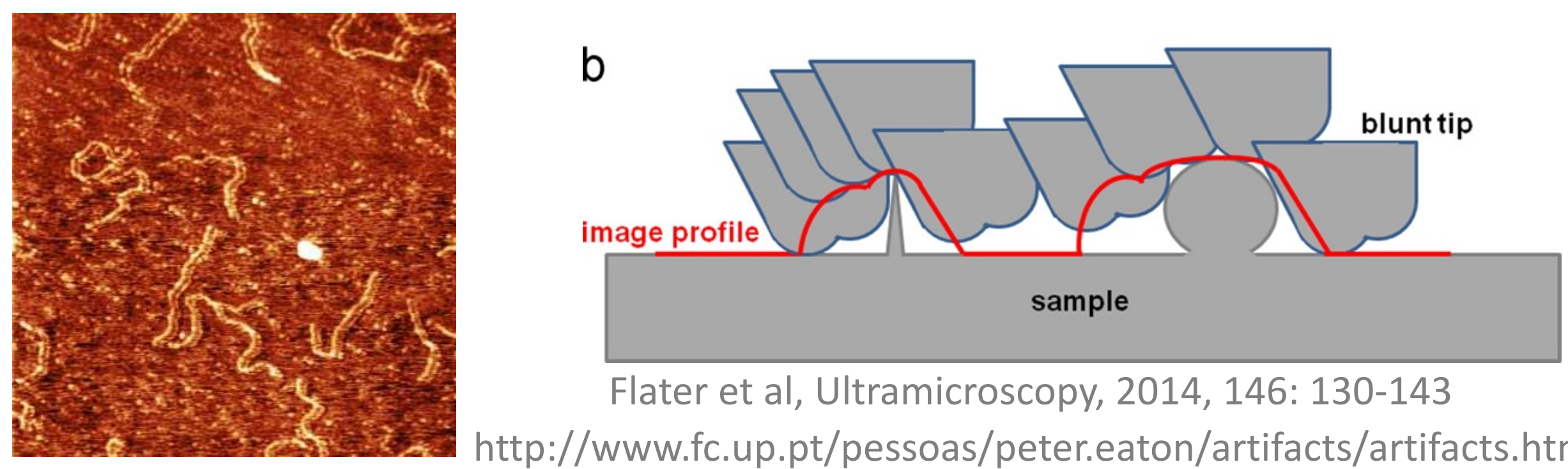

Flater et al, Ultramicroscopy, 2014, 146: 130-143

http://www.fc.up.pt/pessoas/peter.eaton/artifacts/artifacts.html
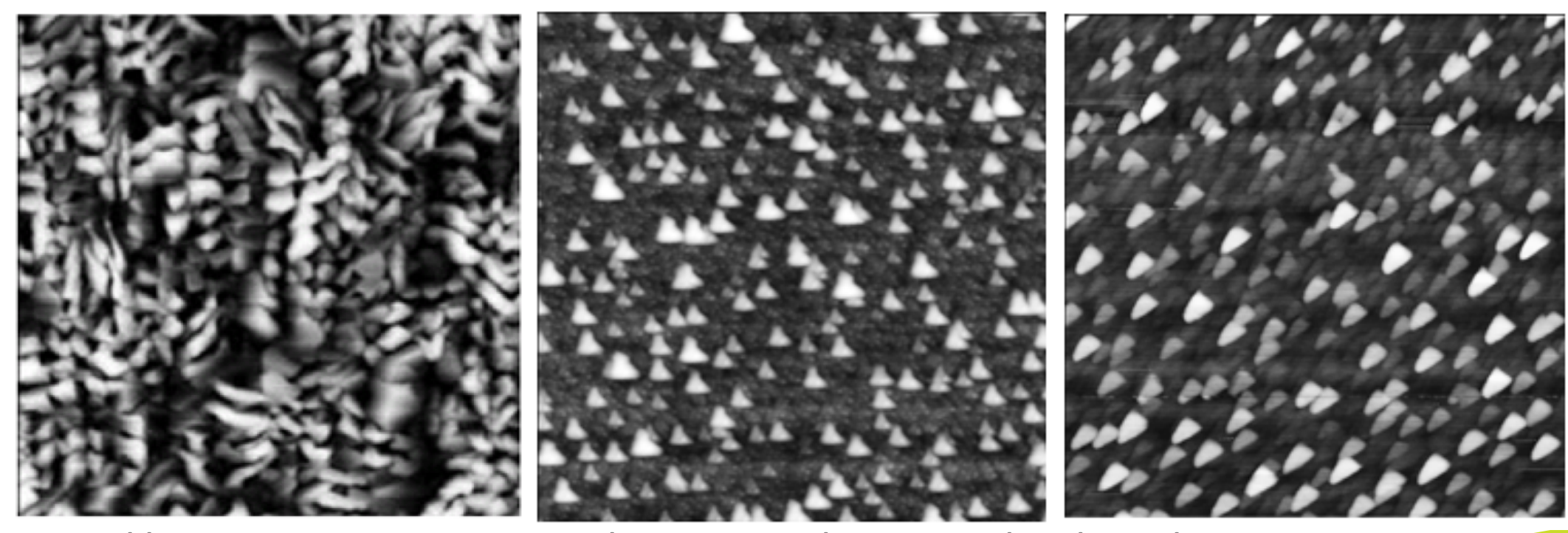

http://www.nanophys.kth.se/nanophys/facilities/nfl/afm/ 


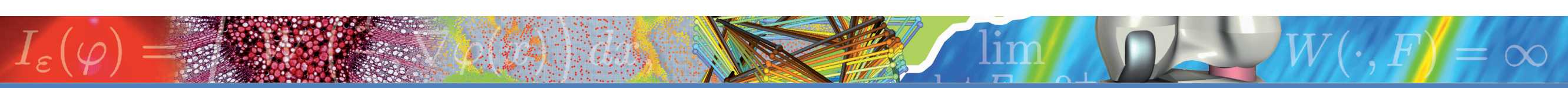

\section{AFM artifacts}

- Images dilation due to tip contamination

- Optical interference
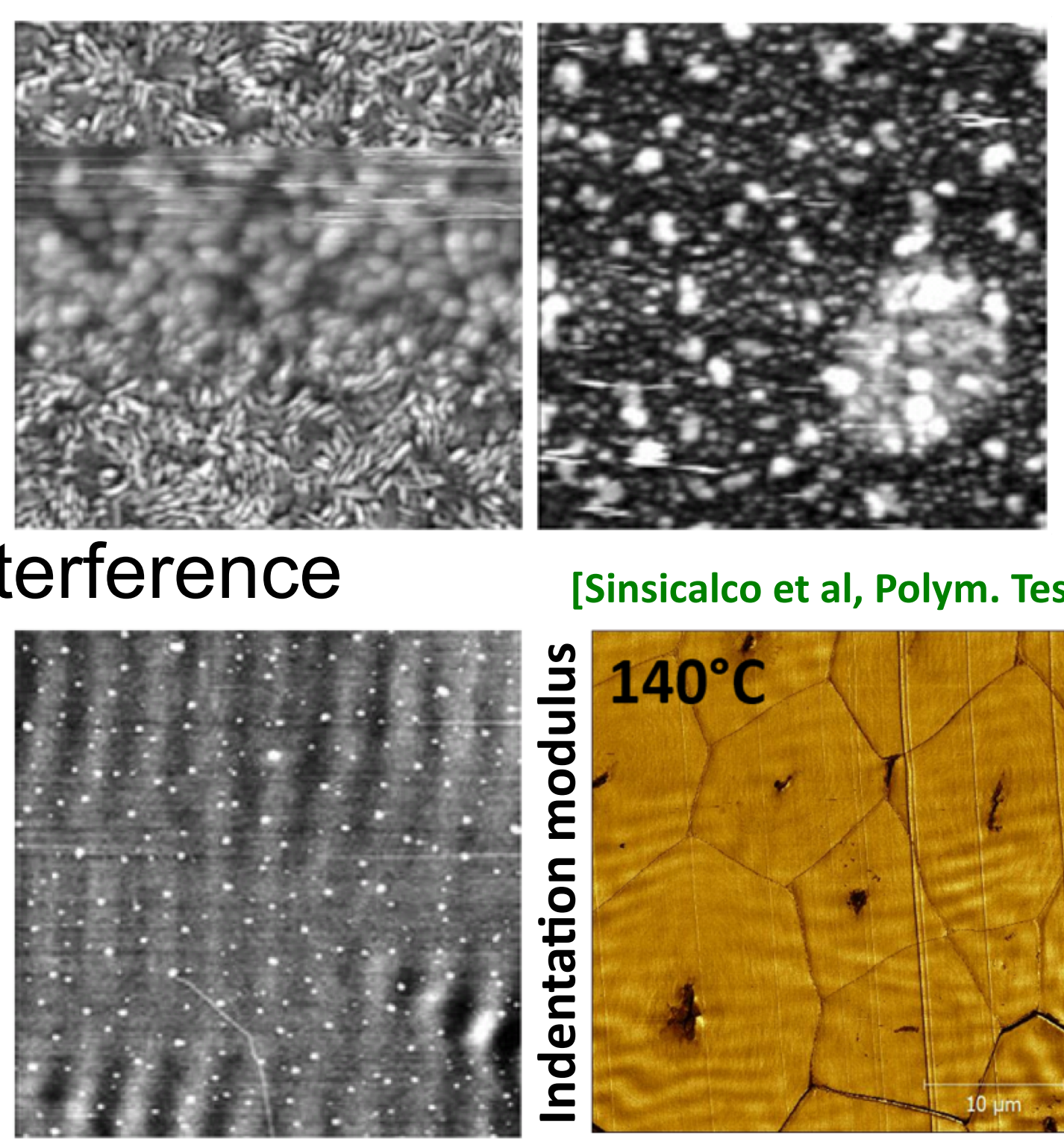

[Sinsicalco et al, Polym. Test., 2018, 69:91-99]
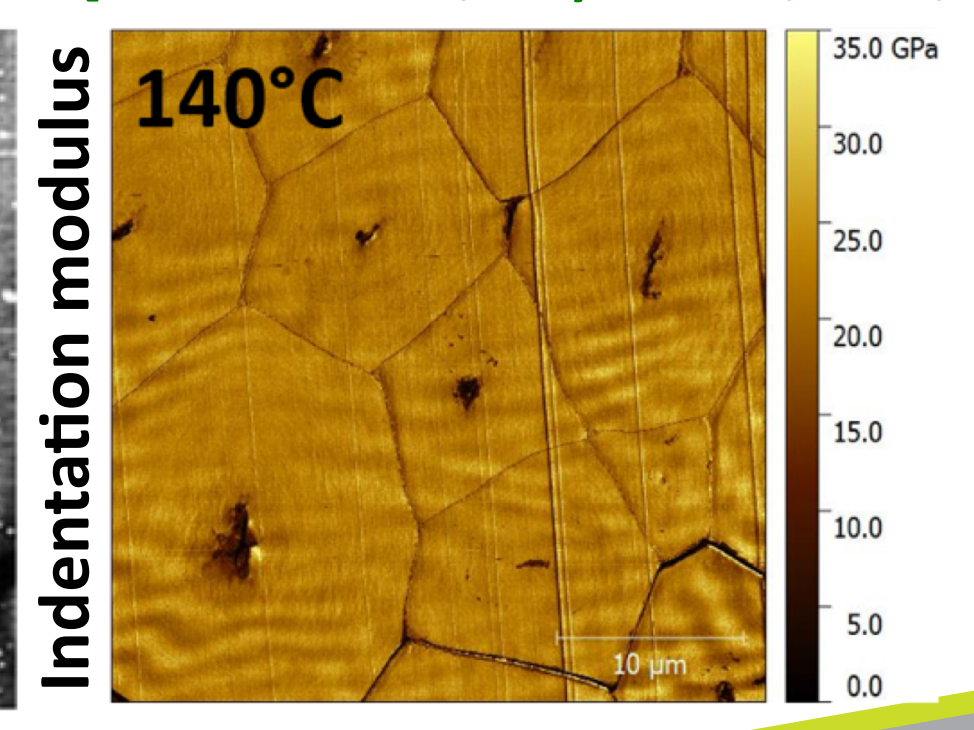


\section{AFM artifacts}

- Distortion due to non-linear piezo-scanner response + aging/creep

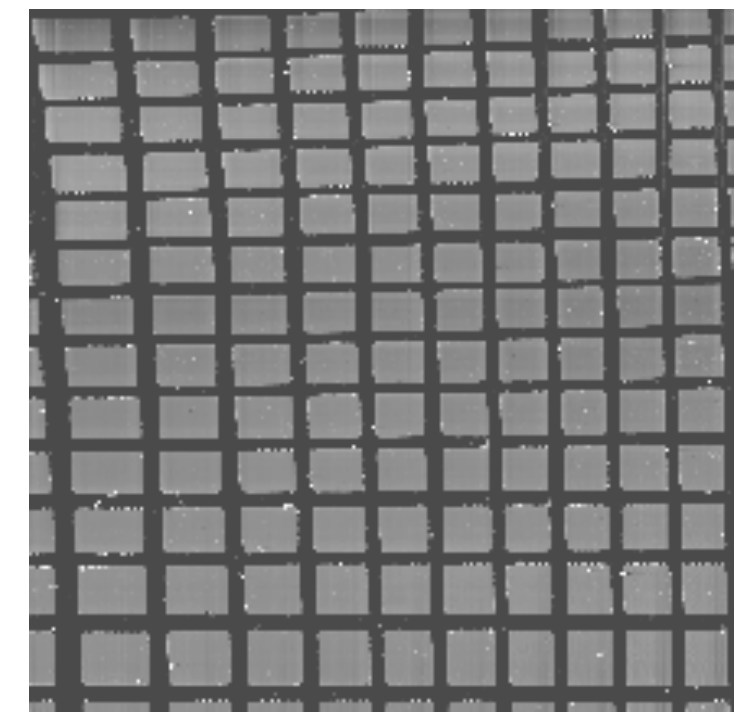

- Background bow/tilt

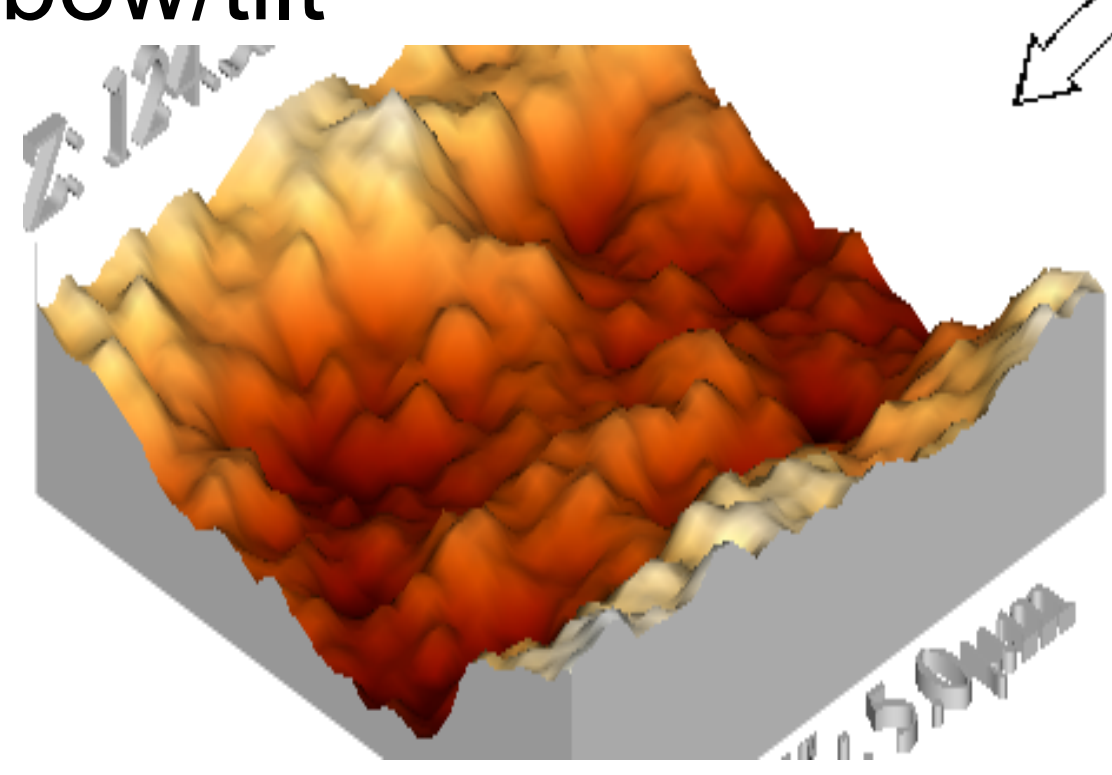




\section{Outline}

- Brief history and basic principles of scanning probe microscopy

- AFM principle and typical set-up

- Tip probe-sample surface interactions

- Tip and cantilever

- Deflection sensor and scanner

- Whole set-up

○ Contact mechanics

- Operating modes

- Contact

- Intermittent

- Force-distance curve and mechanical characterisation modes

- Image analysis and artefacts

\section{Sample preparation}




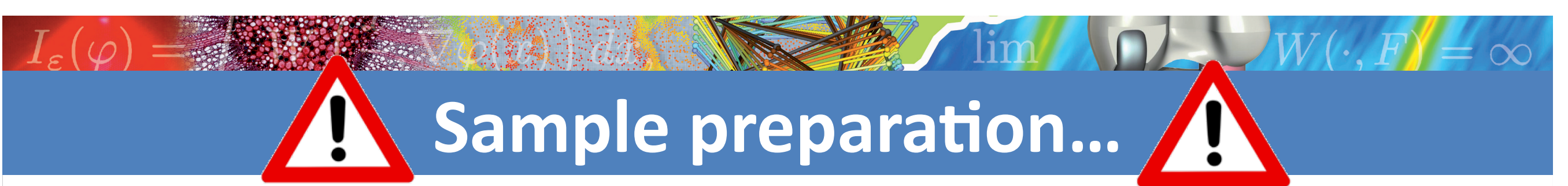

Without embbeding in a resin: no sample modifications but \pm measurement articfacts

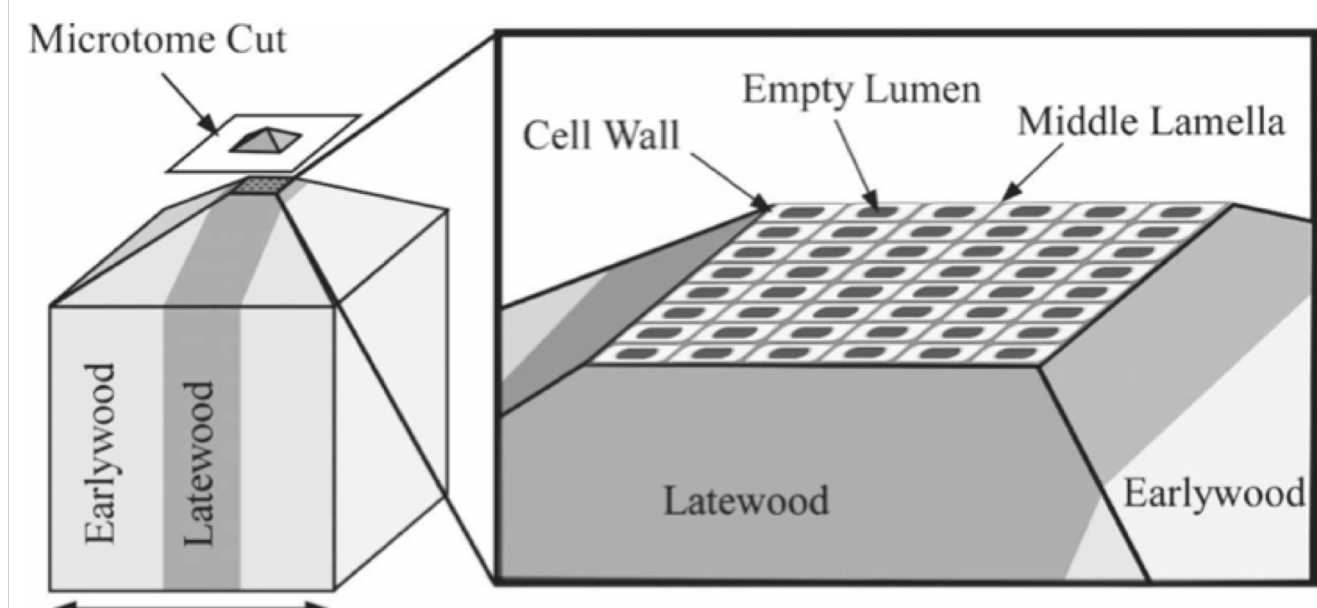

$10 \mathrm{~mm}$

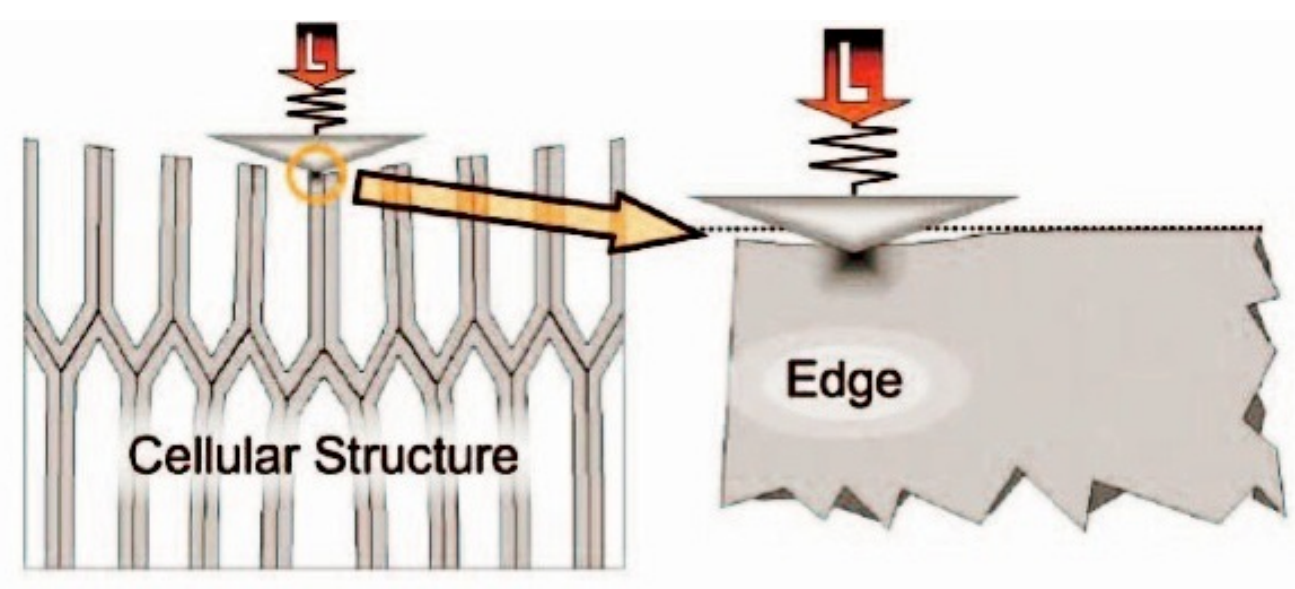

[Jakes et al, J. Mater. Res., 2008] and topography...

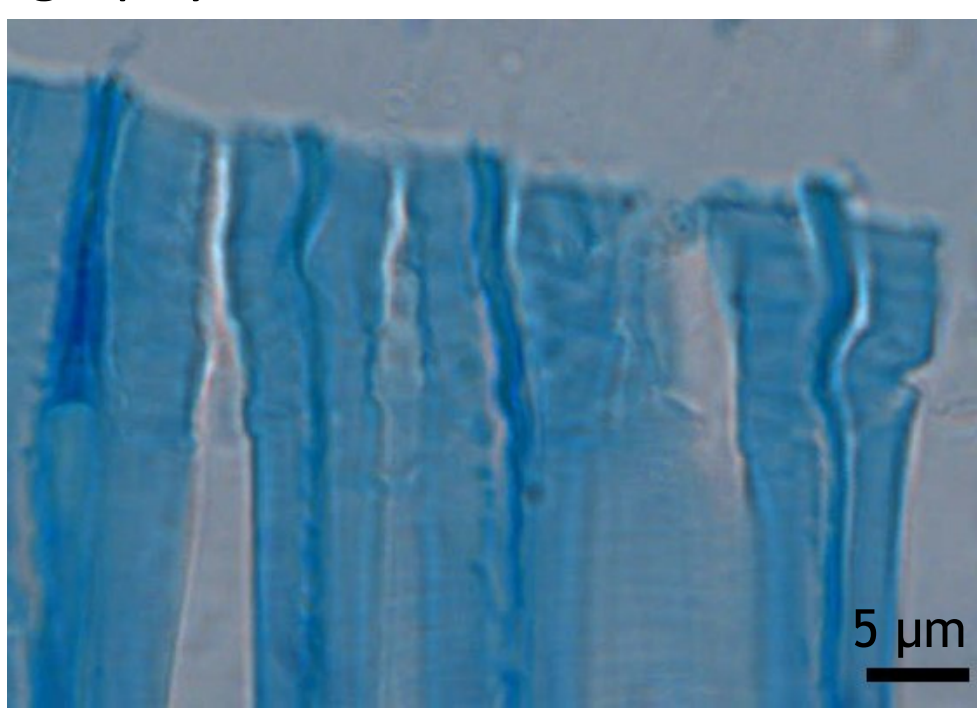

[Clair, Gril et al, IAWA J., 2005]
Dehydration in ethanol and embedding in resin (LR-White, SPURR, ...) or PEG

\pm no thermo-chemical modification of the cell wall (extractives...)?

Resin penetration / inclusion time? Cell wall water content?

Microtome/Grinding/FIB...??!! 


\section{Sample surface effect}

\section{- Effect of sample local slope}

[Heinze et al, Ultramicroscopy, 2018, to appear...]

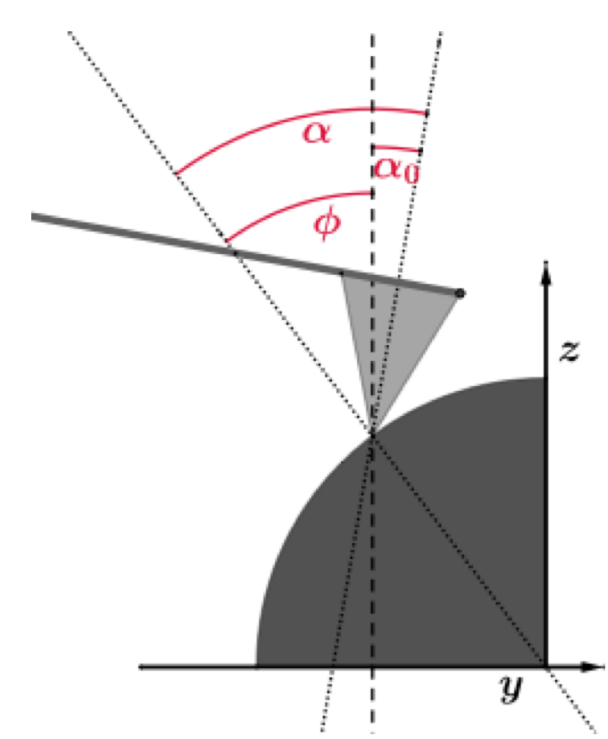

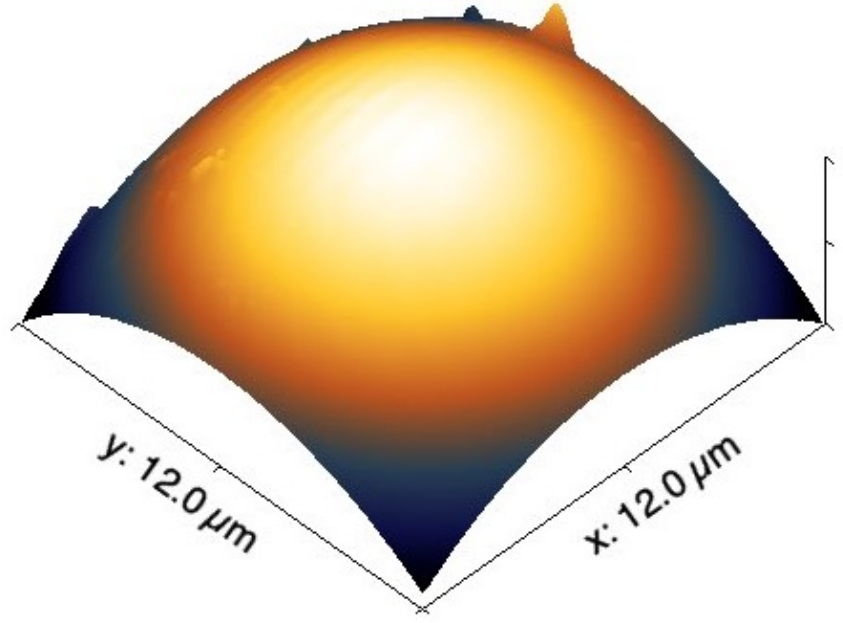

Topography

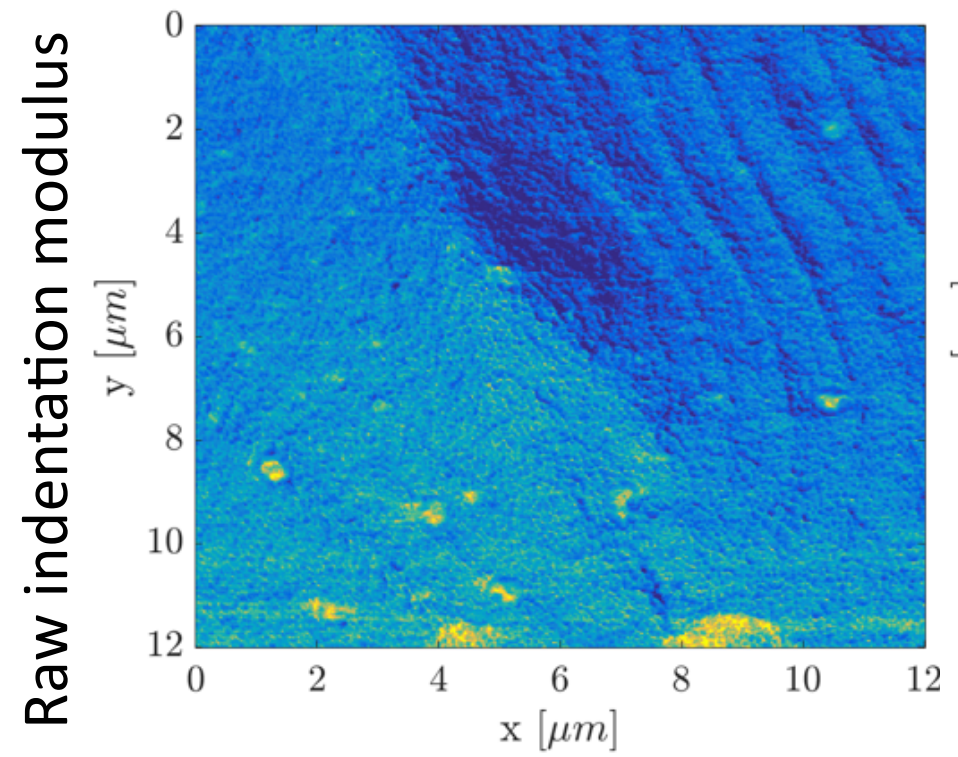

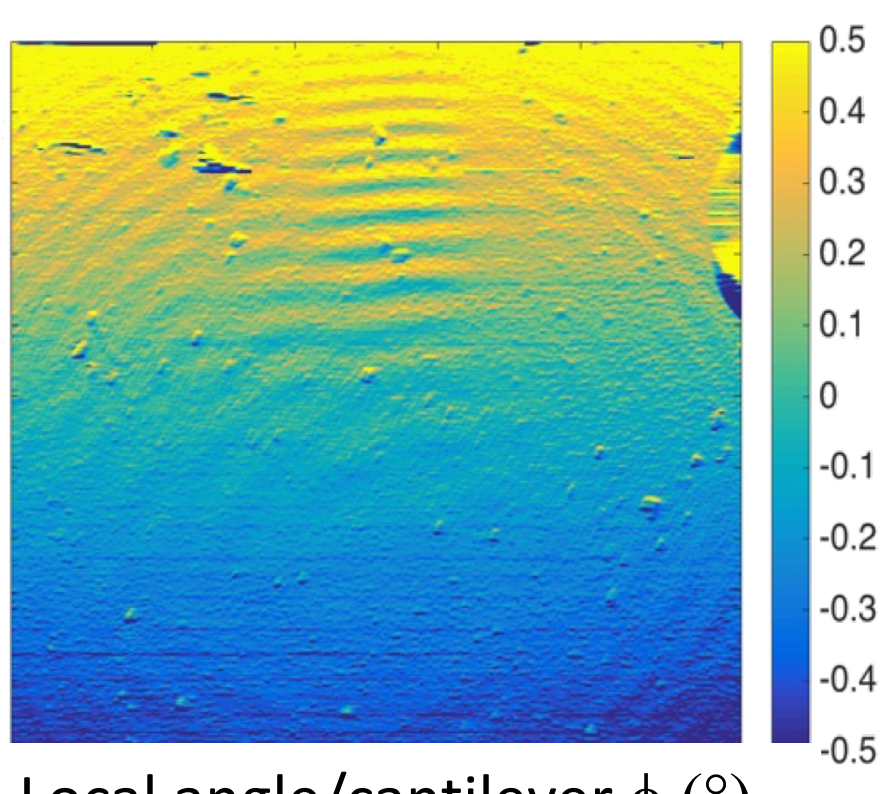

Local angle/cantilever $\phi\left(^{\circ}\right)$
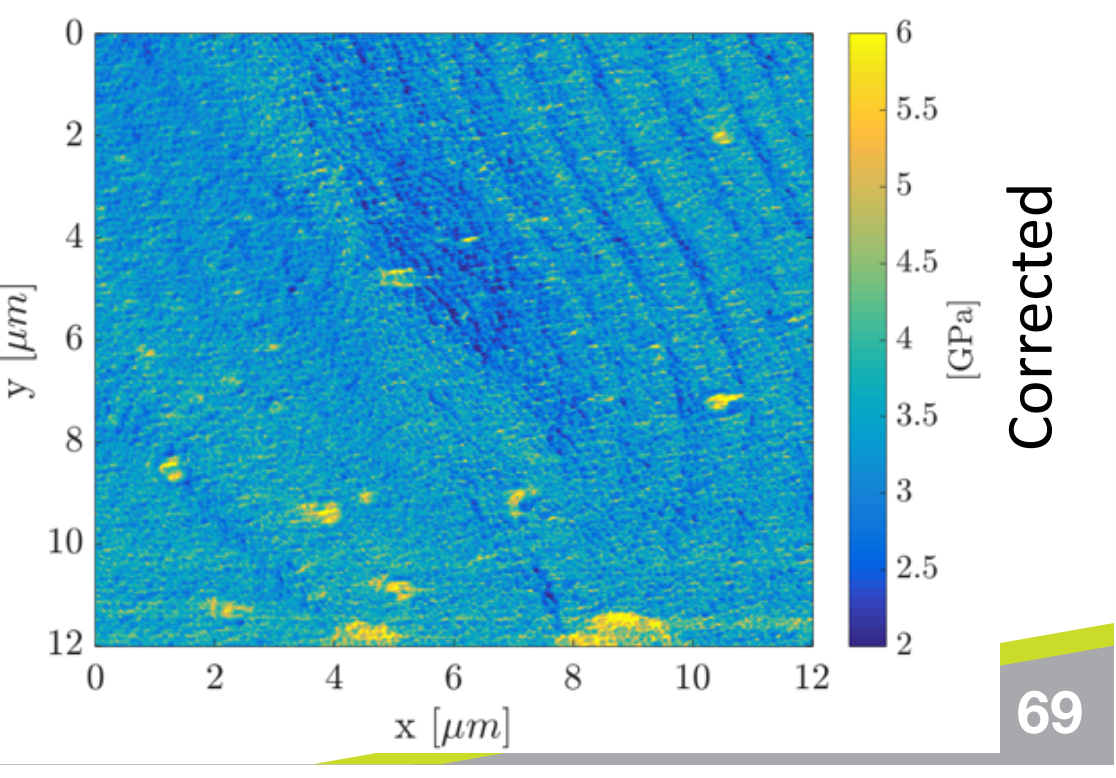


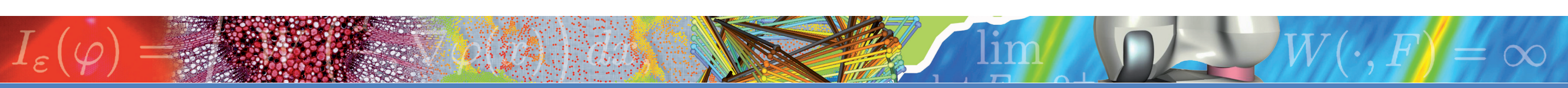
Sample surface effect

\section{- Effect of sample roughness}

Stan and Cook, Nanotechnology, 2008, 19, 235701

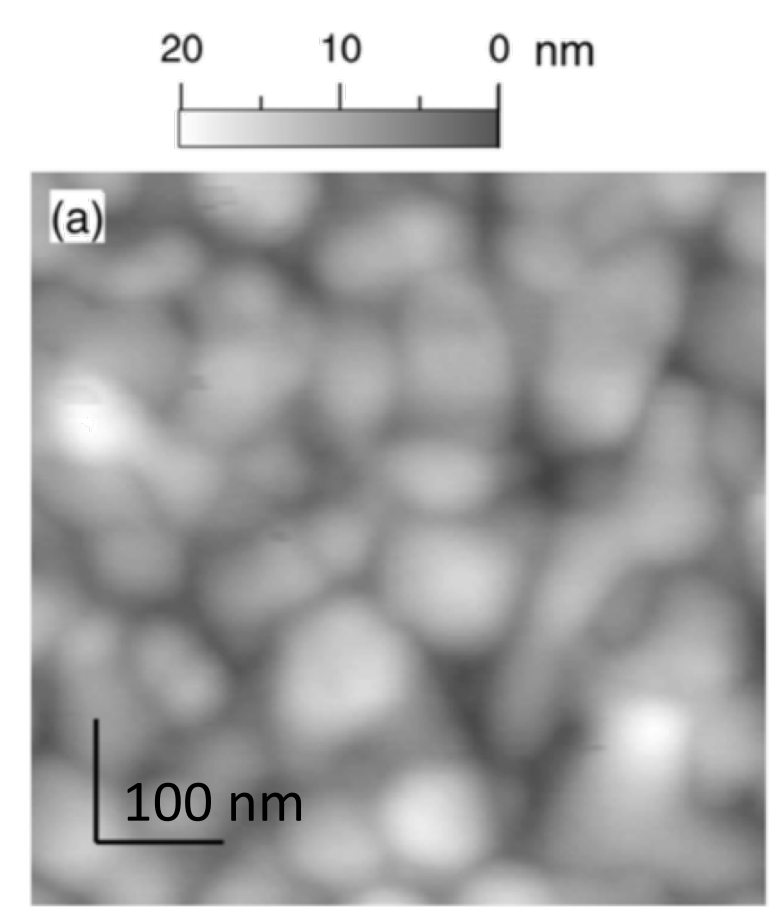

AFM topography

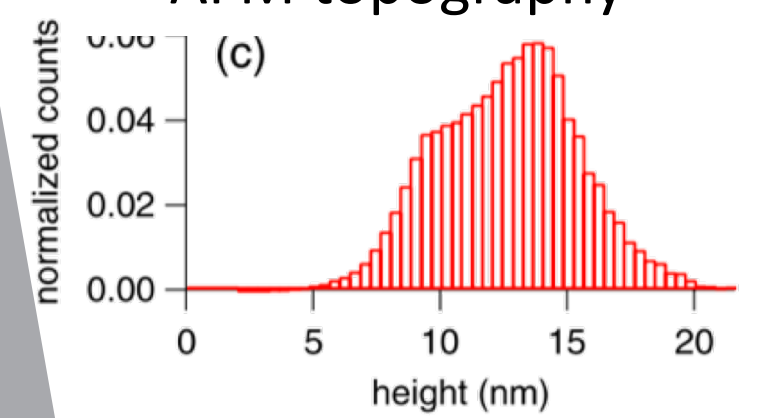

$490 \quad 495 \quad 500 \quad 505 \quad 510 \quad k H z$
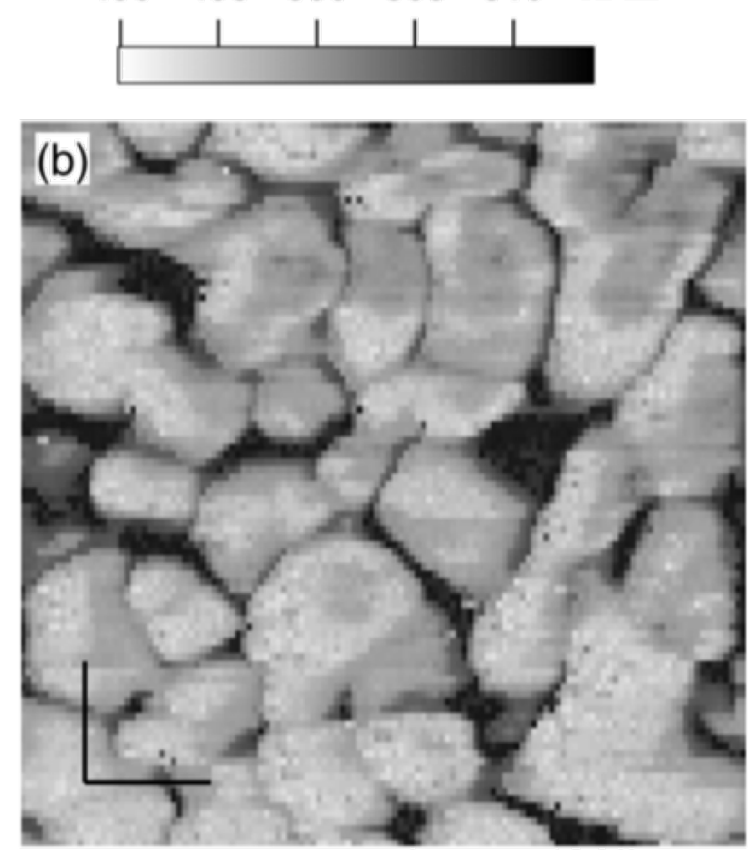

CR-AFM $1^{\text {st }}$ freq

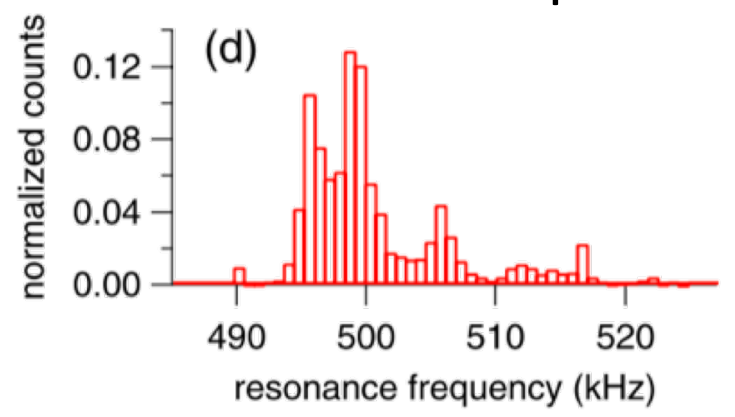

Corrected indentation modulus
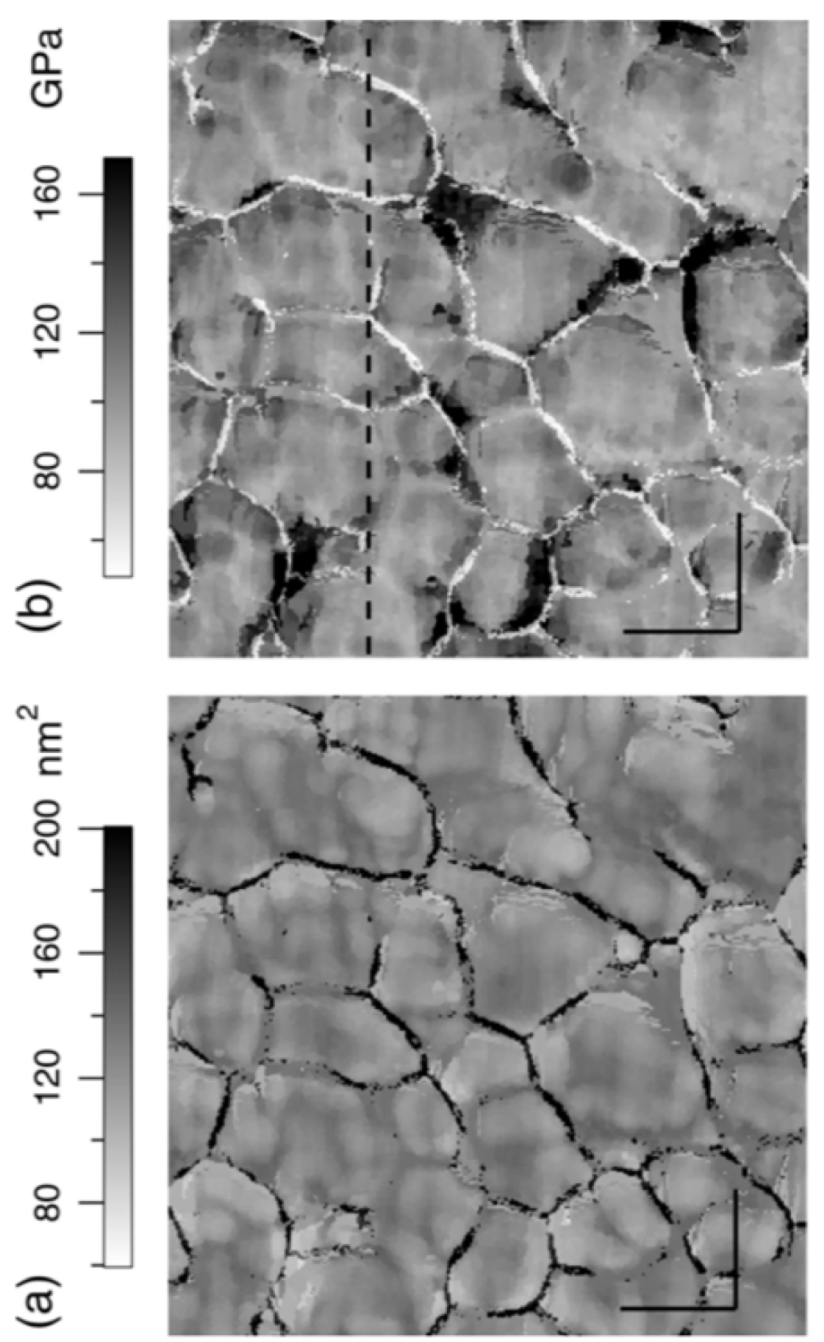

Tip-sample contact area

Au film $\approx\langle 110\rangle, \mathrm{k}_{\mathrm{c}}=14.8 \pm 0.5 \mathrm{~N} / \mathrm{m}, 350 \mathrm{nN}$ 